\title{
Context-Aware Smart Home Monitoring Through the Analysis of Pressure Sequences
}

\author{
by
}

\author{
Amaya Arcelus, M.Sc. E., B.Sc. E.
}

A thesis submitted to the Faculty of Graduate Studies and Research in partial fulfillment of the requirements for the degree of

\section{Doctor of Philosophy in Electrical and Computer Engineering}

\author{
Ottawa-Carleton Institute for Electrical and Computer Engineering \\ Department of Systems and Computer Engineering \\ Carleton University \\ Ottawa, Ontario, Canada, K1S 5B6
}

September 2010

(c) Copyright 2010 Amaya Arcelus 


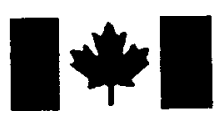

\author{
Library and Archives \\ Canada \\ Published Heritage \\ Branch \\ 395 Wellington Street \\ Ottawa ON K1A ON4 \\ Canada
}

Bibliotheqque et

Archives Canada

Direction du

Patrimoine de l'édition

395 , rue Wellington

Ottawa ON K1A ON4

Canada
Your file Votre référence
ISBN: $978-0-494-70543-8$
Our file Notre référence
ISBN: $978-0-494-70543-8$
NOTICE:

The author has granted a nonexclusive license allowing Library and Archives Canada to reproduce, publish, archive, preserve, conserve, communicate to the public by telecommunication or on the Internet, loan, distribute and sell theses worldwide, for commercial or noncommercial purposes, in microform, paper, electronic and/or any other formats.

The author retains copyright ownership and moral rights in this thesis. Neither the thesis nor substantial extracts from it may be printed or otherwise reproduced without the author's permission.

\section{AVIS:}

L'auteur a accordé une licence non exclusive permettant à la Bibliothèque et Archives Canada de reproduire, publier, archiver, sauvegarder, conserver, transmettre au public par télécommunication ou par l'Internet, prêter, distribuer et vendre des thèses partout dans le monde, à des fins commerciales ou autres, sur support microforme, papier, électronique et/ou autres formats.

L'auteur conserve la propriété du droit d'auteur et des droits moraux qui protège cette thèse. $\mathrm{Ni}$ la thèse ni des extraits substantiels de celle-ci ne doivent être imprimés ou autrement reproduits sans son autorisation.
In compliance with the Canadian Privacy Act some supporting forms may have been removed from this thesis.

While these forms may be included in the document page count, their removal does not represent any loss of content from the thesis.
Conformément à la loi canadienne sur la protection de la vie privée, quelques formulaires secondaires ont èté enlevés de cette thèse.

Bien que ces formulaires aient inclus dans la pagination, il n'y aura aucun contenu manquant.

\section{Canadä}




\section{Abstract}

As the senior population in Canada ages, the need for costly healthcare monitoring increases and the expected workloads of many of the hospitals and nursing care facilities across the country begin to exceed the personnel available. There is therefore an increasing interest in smart home technology which can provide automated monitoring of the health and well-being of seniors. This thesis presents the design of a smart home system which uses unobtrusive pressure sensing and incorporates context-awareness in the monitoring of an occupant's functional mobility. To validate the design, clinical trials were conducted with both healthy adults and mobility-impaired seniors, thereby simulating the decline in an occupant's mobility over an extended period of time due to underlying conditions.

The proposed system design includes several algorithms to extract features from pressure sequences recorded during sit-to-stand ( $\mathrm{SiSt}$ ) transfers. The first was designed to detect and characterize regions of interest from pressure images recorded from sensors placed under a bed mattress. The algorithm allowed the observation of mobility-impaired cases as well as the automated classification of symmetry with an accuracy of $93 \%$. An algorithm was also designed to analyze the sagittal center of pressure trajectory during SiSt transfers from a bed. This allowed the extraction of significant clinical features and enabled the automated detection of mobility impairment with an accuracy of $92 \%$. A 
third algorithm measured the duration of the SiSt transfer using both bed and floor pressure sequences. The durations displayed significant differences between the healthy (2.31 s for young adults, $2.88 \mathrm{~s}$ for seniors) and mobility-impaired (3.57 s for post-stroke, $5.35 \mathrm{~s}$ for post-hip-fracture) participant groups. These results compared closely to those found in recently published literature using more obtrusive methods of data collection.

Various sources of context-awareness were introduced into the monitoring system design including context from pressure sensors embedded in the grab bars of a lavatory commode. A simulated context-aware environment was designed to demonstrate the function of the monitoring system using sequences collected from participants during the various clinical trials. The environment illustrated the effects of incorporating contextawareness through simulated scenarios of occupant behaviour and samples of corrected classification. 


\section{Acknowledgments}

First and foremost I would like to express my sincere gratitude and appreciation to my supervisors Dr. Rafik Goubran and Dr. Frank Knoefel for their technical guidance, professional mentoring and constant support throughout this thesis. I would also like to acknowledge the financial support from the Ontario Graduate Scholarship in Science and Technology (OGSST) and Carleton University.

Thanks are due to Dr. Heidi Sveistrup, Dr. Martin Bilodeau and the students of the School of Rehabilitation Sciences at the University of Ottawa for sharing their clinical expertise and for their collaboration throughout various research projects in the past four

years; to Coleen Kornelsen, Jennifer Poll and Jodie Taylor for their administrative support at Carleton University and at the Élisabeth Bruyère Hospital; and to the members of the Digital Signal Processing lab at Carleton University, especially Megan Holtzman, Idana Veledar, Christophe Herry, Anneliis Tosine and Vilas Joshi for their sharing of knowledge, experience and friendship.

Finally, my heartfelt personal thanks go to Andrew Feltham for his unwavering patience, support, and encouragement in this process as in all of my endeavours; and to Dr. Francisco Arcelus and Dr. Maria Arcelus for providing the foundation and being my continuous role models in the pursuit of academic, professional and personal excellence. 


\section{Table of Contents}

Abstract

Acknowledgments $\quad$ v

Table of Contents $\quad$ vi

List of Tables $\quad$ xi

List of Figures $\quad$ xii

List of Acronyms $\quad$ xvii

List of Symbols $\quad$ xviii

$\begin{array}{ll}\text { Chapter 1: Introduction } & 1\end{array}$

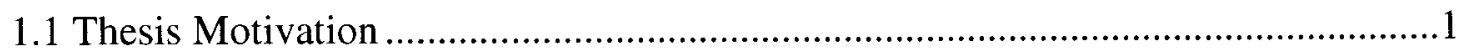

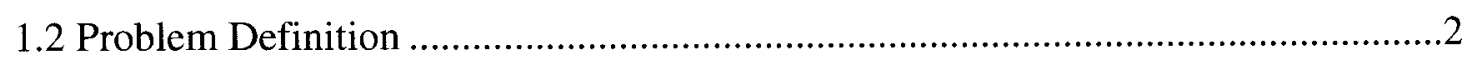

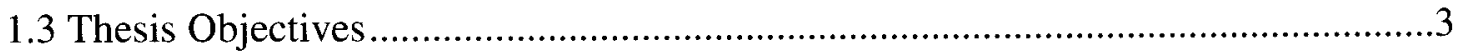

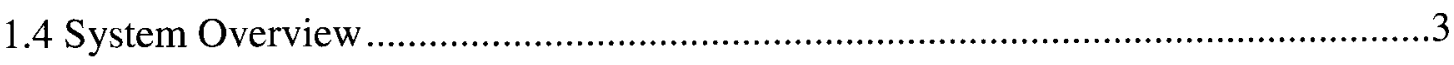

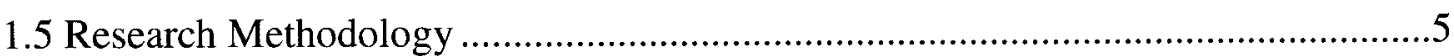

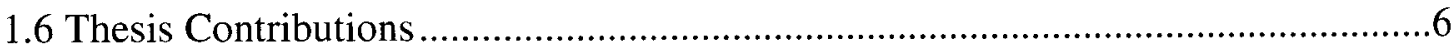

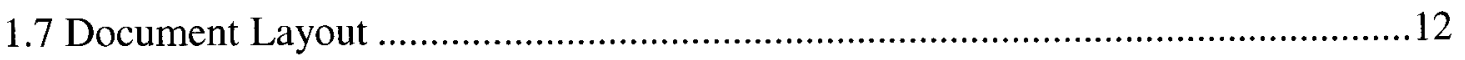

Chapter 2: Background Review 13

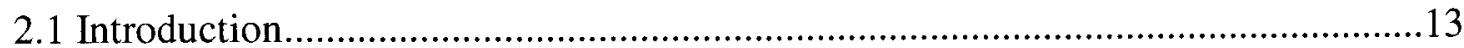

2.2 Context-Awareness in the Smart Home Environment........................................13

2.2.1 General Purpose Smart Homes ................................................................... 14

2.2.2 Health Monitoring Smart Homes............................................................... 14

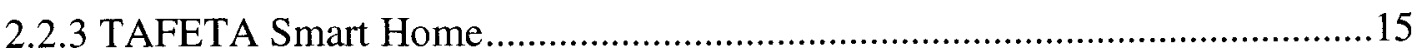




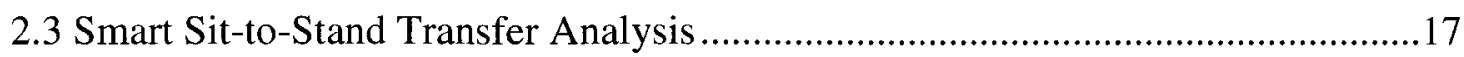

2.3.1 Image Processing .........................................................................................

2.3.2 Center of Pressure Trajectories....................................................................18

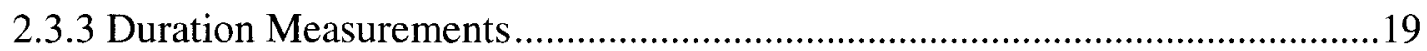

2.4 Pressure Sensing Within the Smart Home ............................................................20

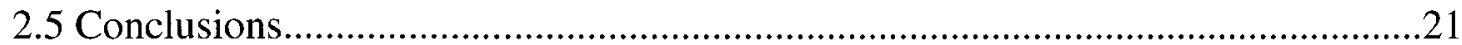

Chapter 3: Experimental Setup and Clinical Data Collection 23

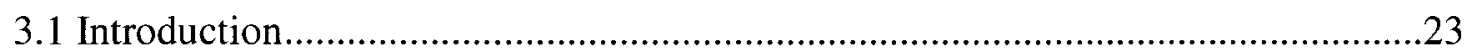

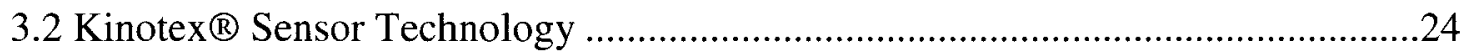

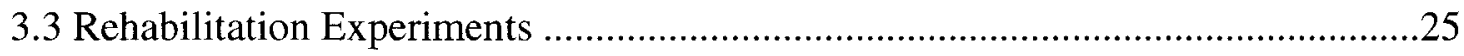

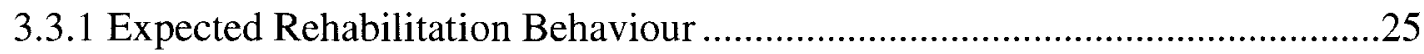

3.3.2 Actual Rehabilitation Behaviour .................................................................26

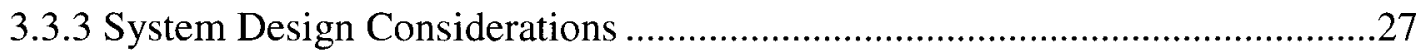

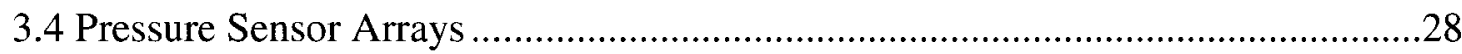

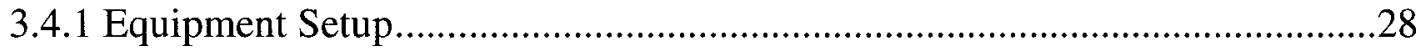

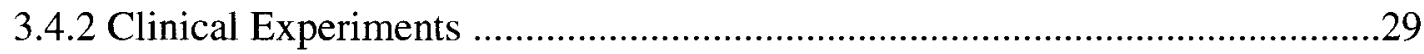

3.4.3 Video Verification Protocol.............................................................................. 31

3.5 Pressure-Sensitive Grab Bars ............................................................................

3.5.1 Grab Bar Instrumentation ........................................................................

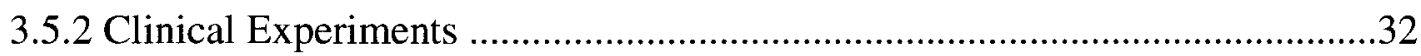

Chapter 4: Region of Interest Detection and Characterization from Pressure Image Sequences $\quad 34$

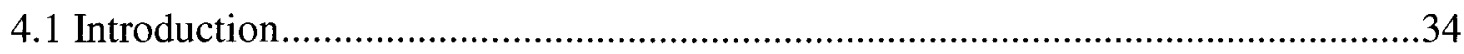

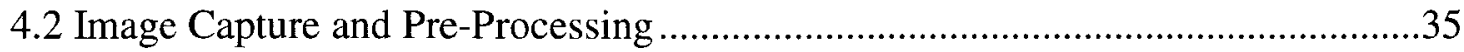

4.3 Region of Interest Detection ................................................................................

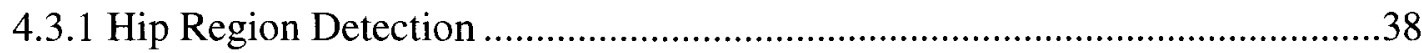

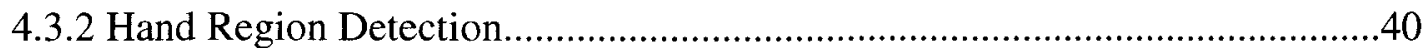

4.4 Region of Interest Pressure Sequences and Feature Extraction.................................44 
4.4.1 Regional Pressure Sequences .........................................................................44

4.4.2 Clinical Feature Definition ...........................................................................46

4.4.3 Interactive Clinical Assessment Tool ..............................................................47

4.4.4 Symmetry Measurement .................................................................................49

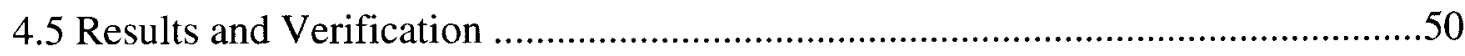

4.5.1 Abnormal Transfer Visibility ......................................................................50

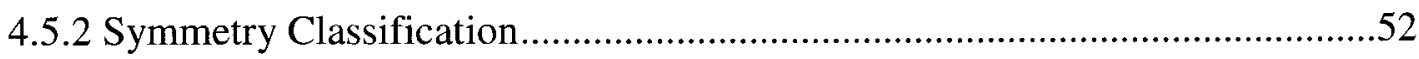

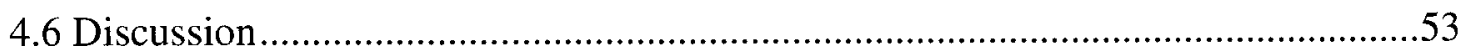

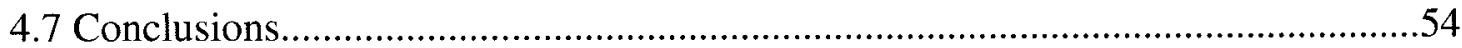

Chapter 5: Sagittal Center of Pressure Trajectory Analysis from Bed Pressure Sequences $\quad 55$

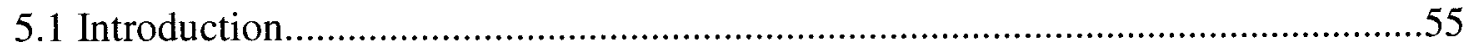

5.2 Properties of the Center of Pressure Trajectory During Sit-to-Stand .......................56

5.3 Center of Pressure Sequences and Feature Extraction ............................................57

5.3.1 Sagittal Trajectory Sequences...........................................................................58

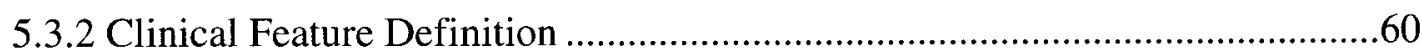

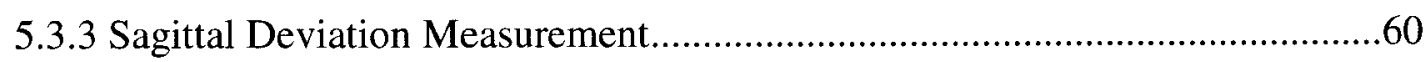

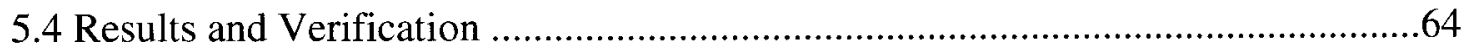

5.4.1 Extracted Clinical Features ..............................................................................64

5.4.2 Relative Significance of Extracted Clinical Features ........................................66

5.4.3 Sagittal Deviation Classification ....................................................................68

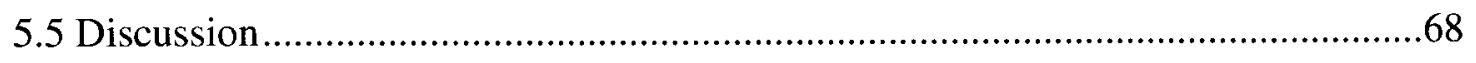

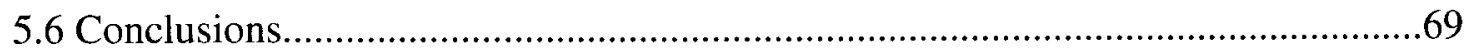

\section{Chapter 6: Transfer Duration Measurements from Bed and Floor Pressure}

$\begin{array}{ll}\text { Sequences } & \mathbf{7 0}\end{array}$

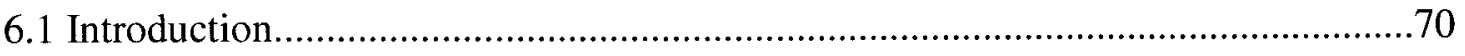

6.2 Bed and Floor Pressure Sequences .................................................................

6.3 Start of Sit-to-Stand Transfer on the Bed .............................................................

viii 
6.3.1 Center of Pressure Sequence …………………...........................................73

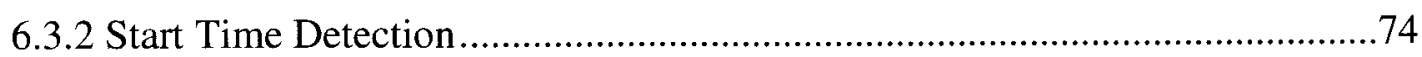

6.4 End of Sit-to-Stand Transfer on the Floor ............................................................75

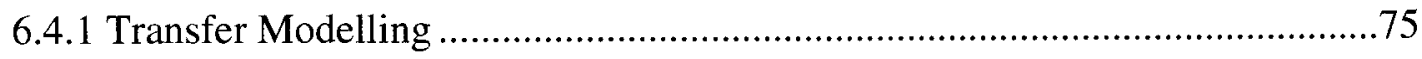

6.4.2 End Time Detection .................................................................................78

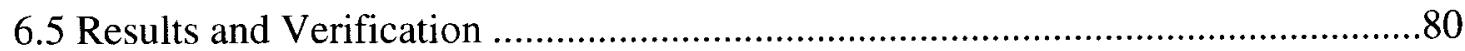

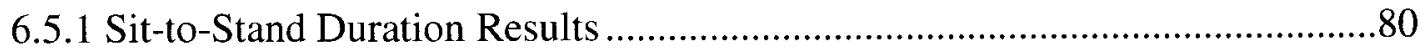

6.5.2 Duration of Intermediate Phases ..................................................................82

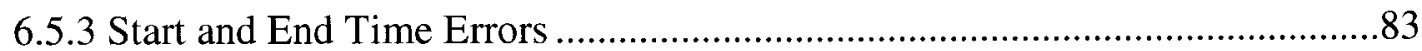

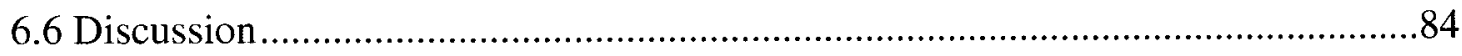

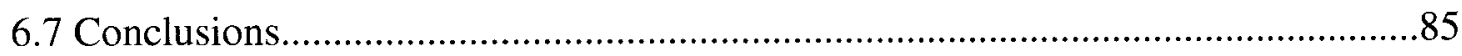

Chapter 7: Context in Mobility Monitoring $\quad 86$

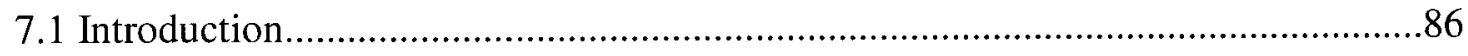

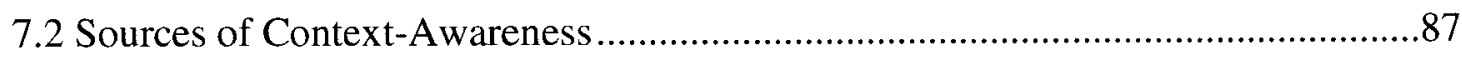

7.2.1 Human Context - Manual Information Entry ...................................................87

7.2.2 Derived Context - Electronic Health Records .....................................................87

7.2.3 Sensed Context - Pressure-Sensitive Grab Bars ...............................................8

7.3 Sensed Context Using Pressure-Sensitive Grab Bar Sequences................................88

7.3.1 Sensor Calibration and Output Estimation .......................................................89

7.3.2 Grab Bar Sequences and Feature Extraction .................................................92

7.3.3 Grab Bar Usage Results and Verification........................................................95

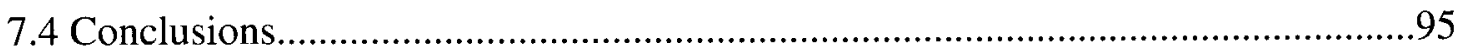

Chapter 8: Context-Aware Smart Home Monitoring $\quad 97$

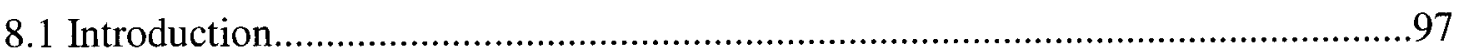

8.2 Simulated Context-Aware Environment.............................................................98

8.3 Classification of Occupant Mobility Based on Feature Extraction Algorithms and

Context Provided by Pressure-Sensitive Grab Bars .....................................................99

8.3.1 Scenario 1: Normal Behaviour ..................................................................100 
8.3.2 Scenario 2: Abnormal Behaviour .

103

8.4 Classification of Occupant Mobility Using Algorithm Selection and Context-

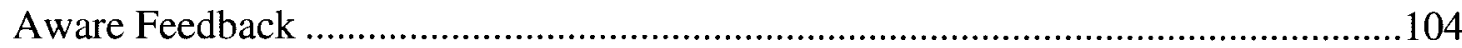

8.4.1 Context-Aware Algorithm Selection ...........................................................104

8.4.2 Context-Aware Feedback Samples ..................................................................106

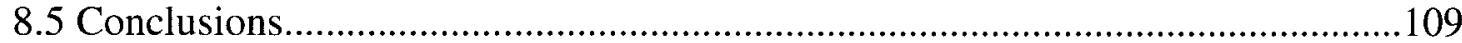

$\begin{array}{ll}\text { Chapter 9: Conclusions } & 110\end{array}$

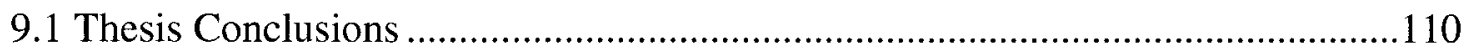

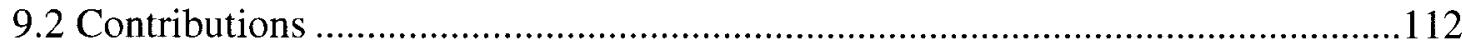

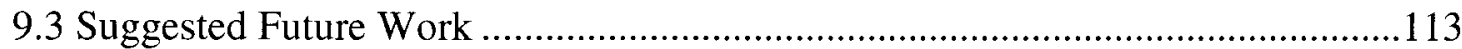

$\begin{array}{ll}\text { References } & 115\end{array}$ 


\section{List of Tables}

Table 4.1: Results of symmetry classification during SiSt transfers .............................53

Table 5.1: Results of sagittal CP features extracted from SiSt transfers ........................65

Table 5.2: Results of stepwise binary logistic regression for clinical features.................67

Table 5.3: Results of impairment classification during SiSt transfers...........................68

Table 6.1: Video-based (VB) and pressure-based (PB) SiSt duration times ...................80

Table 7.1: Clinical features extracted from the StSi transfer of a senior participant.........95 


\section{List of Figures}

Figure 1.1: Traditional monitoring system................................ 2

Figure 1.2: Monitoring system with three feature extraction algorithms to observe significant clinical features.................................. 4

Figure 1.3: Monitoring system with introduction of context to select appropriate algorithms....................................................... 4

Figure 1.4: Monitoring system with introduction of feedback loop to provide context-aware feedback to selector and algorithms.................. 5

Figure 2.1: Views of the bedroom, lavatory and kitchen in the TAFETA 'Independent Living Apartment' laboratory, located in the Élisabeth Bruyère Hospital in Ottawa, ON, Canada............................ 16

Figure 3.1: Monitoring system data collection................................ 24

Figure 3.2: Kinotex ${ }^{\circledR}$ pressure sensor (a) top view of a Single Point sensor and (b) side view schematic of the sensor components...................... 24

Figure 3.3: Expected bed-exit times for a rehabilitation patient.................... 26

Figure 3.4: Actual bed-exit times for rehabilitation patient $\# 1 \ldots \ldots \ldots \ldots \ldots \ldots \ldots \ldots . . \ldots$

Figure 3.5: Actual bed-exit times for rehabilitation patient $\# 2 \ldots \ldots \ldots \ldots \ldots \ldots \ldots \ldots . \ldots . \ldots . \ldots$

Figure 3.6: Large Sensor Array by Tactex Controls Inc........................... 28

Figure 3.7: Pressure sensor arrays (a) pressure-sensitive bed mats and (b) pressuresensitive floor plate.............................................. 29

Figure 3.8: Transfer sequence from lying supine to standing performed by experiment participants during data collection...................... 30

Figure 3.9: Commode grab bars instrumented with embedded pressure sensors....... 32

Figure 4.1: Monitoring system feature extraction algorithm A................... 35 
Figure 4.2: Ideal image sequence of SiSt transfer during bed-exit.................. 36

Figure 4.3: System architecture of the ROI detection and feature extraction from bed pressure image sequences.................................... 38

Figure 4.4: Image $I M G_{\text {sit }}$ of a healthy participant sitting upright................ 39

Figure 4.5: Binary hip mask HipM of a healthy participant............................. 39

Figure 4.6: Sample of sequential frame analysis for a healthy participant (a) image in current frame $I M G(f)$, (b) image in subsequent frame $I M G(f+1)$, (c) image of decreasing pixels $I M G_{d e c}(f)$, and (d) amplified image of

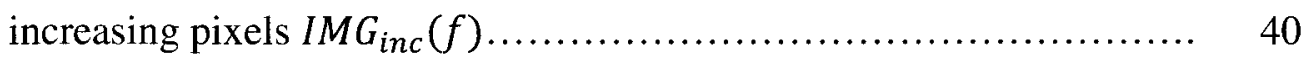

Figure 4.7: Binary image $I M G_{R E G}(f)$ containing regions $R E G(f, 1)$ and $R E G(f, 2) \quad 41$

Figure 4.8: $I M G_{\text {sit }}$ with corresponding hip parameters and hand region boundaries. 43

Figure 4.9: $I M G_{R E G}(f)$ displaying samples of regions outside of hand region

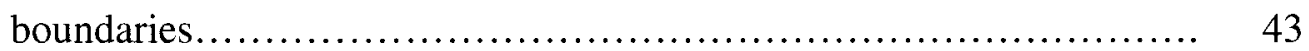

Figure 4.10:Grayscale hand mask $\operatorname{Hand} M$ of a healthy participant....................... 44

Figure 4.11:Healthy transfer pressure sequences from (a) hip region and (b) hand regions............................................................. 45

Figure 4.12:Mobility-impaired transfer pressure sequences from (a) hip region and

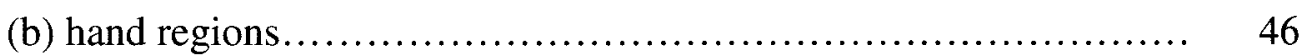

Figure 4.13:SiSt clinical assessment tool prototype: transfer analysis view containing image sequence playback, regional pressure sequences and extracted features...............................................

Figure 4.14:SiSt clinical assessment tool prototype: trends view containing the option of viewing the last day, week or month of extracted features over time.

Figure 4.15: Selection of symmetry threshold by considering (a) the ROC curve and (b) the distance between $[0,1]$ and the ROC curve.

Figure 4.16: Single-handed transfer pressure sequences from (a) hip region and (b) hand regions. 
Figure 4.17:Bouncing transfer pressure sequences from (a) hip region and (b) hand regions................................................... 52

Figure 5.1: Monitoring system feature extraction algorithm B................. 56

Figure 5.2: SiSt transfer sequence with corresponding pressure images........... 57

Figure 5.3: Measured $C P_{y}(n)$ trajectories during the SiSt of two healthy

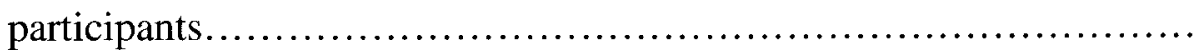

Figure 5.4: Measured $C P_{y}(n)$ trajectory for mobility-impaired participants, exhibiting characteristics of (a) rocking by a participant post-hipfracture and (b) SiSt failure by a participant post-stroke............... 59

Figure 5.5: Simulated $C P_{y}(n)$ trajectory for SiSt transfers of (a) healthy and (b) mobility-impaired participants.................................. 61

Figure 5.6: System architecture of mobility-impaired SiSt transfer classification from bed pressure sequences.................................. 61

Figure 5.7: Measured sequences for a healthy participant (a) $C P_{y}(n)$ before detrending and (b) $C P_{y_{d t r}}(n)$ after detrending........................ 63

Figure 5.8: Measured sequences for a post-hip-fracture participant (a) $C P_{y}(n)$ before detrending and (b) $C P_{y_{d t r}}(n)$ after detrending................. 63

Figure 5.9: Selection of sagittal deviation threshold $v$ thr by considering (a) the ROC curve and (b) the distance between $[0,1]$ and the ROC curve...... 64

Figure 6.1: Monitoring system feature extraction algorithm C.................. 71

Figure 6.2: System architecture of sit-to-stand duration measurements from bed and floor pressure sequences................................ 71

Figure 6.3: Sequences of pressure sums of bed and floor sensors over time......... 72

Figure 6.4: Orientation of bed sensor axes in sit-to-stand duration measurements.. $\quad 73$

Figure 6.5: $C P_{y}(n)$ sequence for five consecutive SiSt transfers................. 74

Figure 6.6: Transfer start detection with delineated sections of I: sitting position, II: SiSt transfer, and III: standing position.......................... 75

Figure 6.7: Normalized sum of floor pressure for five consecutive SiSt transfers.... 76 xiv 
Figure 6.8: Modelled SiSt transfer from floor data with delineated sections of I: end of previous stand-to-sit (StSi) transfer, II: feet resting on floor plate, III: start of SiSt transfer, IV: modelled part of SiSt transfer, and V: StSi transfer................................................. 78

Figure 6.9: Transfer end detection..................................... 79

Figure 6.10:Pressure-based SiSt duration medians and deviations for healthy and mobility-impaired participants................................... 81

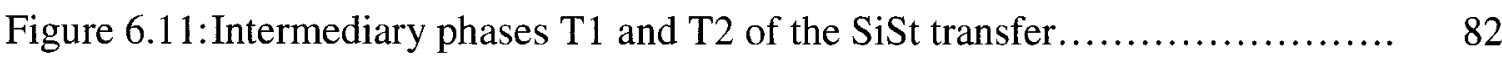

Figure 6.12: Scatter plot of SiSt phase T1 versus T2 for healthy and mobilityimpaired participants............................................. 83

Figure 7.1: Monitoring system context-awareness............................ 86

Figure 7.2: Calibration of embedded pressure sensors.......................... 89

Figure 7.3: $2^{\text {nd }}$-order model fit of force as a function of sensor output............. 90

Figure 7.4: Data sequence of (a) sensor outputs, (b) location estimates and (c) force estimates for StSi and SiSt transfers performed by a young participant... 91

Figure 7.5: Direction of increasing location along the grab bar.................. 92

Figure 7.6: Data sequence of (a) location estimates and (b) force estimates for StSi and SiSt transfers performed by a senior participant................. 93

Figure 7.7: Feature extraction from the sequences of (a) location estimates and (b) force estimates during the StSi transfer of a senior participant

Figure 8.1: Monitoring system algorithm selector, trends, algorithm output fusion and feedback loop........................................ 98

Figure 8.2: Simulated Scenario 1: occupant is classified as being within normal

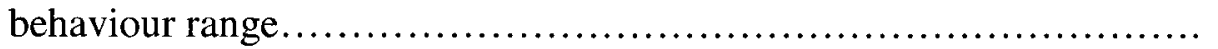

Figure 8.3: Algorithm A - ROI results: normalized pressure sequences during the occupant's SiSt transfers

Figure 8.4: Algorithm B - CP results: center of pressure sequences during the occupant's SiSt transfers displaying (a) expected output (b) actual

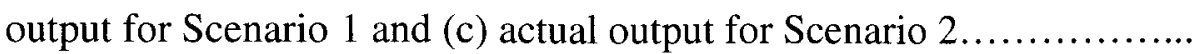


Figure 8.5: Sensed context - pressure-sensitive grab bar results: actual sequences output in Scenario 1: (a) left hand force (b) right hand force (c) left hand location and (d) right hand location.......................... 102

Figure 8.6: Simulated Scenario 2: occupant is classified as experiencing abnormal behaviour and requires further attention...............................

Figure 8.7: Sensed context - pressure-sensitive grab bar results: actual sequences output in Scenario 2: (a) left hand force (b) right hand force (c) left hand location and (d) right hand location...............................

Figure 8.8: Pressure sequences from a healthy senior's SiSt transfer initially misclassified by the ROI algorithm as asymmetrical....................

Figure 8.9: Pressure sequences from a post-stroke patient's SiSt transfer initially output as having used both hands...........................................

Figure 8.10:Pressure sequences from a post-stroke patient's SiSt transfer initially misclassified by the ROI algorithm as symmetrical..................... 


\section{List of Acronyms}

$\begin{array}{ll}\text { ANOVA } & \text { Analysis of Variance } \\ \text { CM } & \text { Center of Mass } \\ \text { CP } & \text { Center of Pressure } \\ \text { CT } & \text { Computed Tomography } \\ \text { ÉBH } & \text { Élisabeth Bruyère Hospital } \\ \text { ÉBRI } & \text { Élisabeth Bruyère Research Institute } \\ \text { EHR } & \text { Electronic Health Record } \\ \text { FIR } & \text { Finite Impulse Response } \\ \text { FMRI } & \text { Functional Magnetic Resonance Imaging } \\ \text { GUI } & \text { Graphical User Interface } \\ \text { LED } & \text { Light Emitting Diode } \\ \text { LH } & \text { Left Hand } \\ \text { PB } & \text { Pressure-Based } \\ \text { PET } & \text { Positron Emission Tomography } \\ \text { RH } & \text { Right Hand } \\ \text { ROC } & \text { Receiver Operating Characteristic } \\ \text { ROI } & \text { Region of Interest } \\ \text { SiSt } & \text { Sit-to-Stand } \\ \text { StSi } & \text { Stand-to-Sit } \\ \text { TAFETA } & \text { Technology Assisted Friendly Environment for the Third Age } \\ \text { VB } & \text { Video-Based }\end{array}$

xvii 


\section{List of Symbols}

$\begin{array}{ll}C P_{\text {sit }}, y & \text { Center of pressure location of the seated region } \\ C P_{x} & \text { x-coordinate of the center of pressure } \\ C P_{y} & \text { y-coordinate of the center of pressure } \\ C P_{y_{d t r}} & \text { Detrended trajectory of the center of pressure's y-coordinate } \\ \text { diffP } & \text { Sequence of the difference between left and right hand pressures } \\ d t h r & \text { Dominant pressure threshold for asymmetry classification } \\ \text { e } & \text { Error function } \\ \mathrm{e}_{\text {min }} & \text { Error function minimized in the least squares sense } \\ f & \text { Frame index } \\ F & \text { Number of frames in the transfer } \\ f_{\text {lin }} & \text { Linear function approximation } \\ F_{\text {sit }} & \text { Number of frames in which the body sits upright } \\ \text { H(s) } & \text { Transfer function in the Laplace domain } \\ \text { HandM } & \text { Hand mask } \\ \text { HandM } & \text { x-coordinate of pixels within the hand mask } \\ \text { HipD } & \text { Hip depth } \\ \text { HipM } & \text { Hip mask } \\ \text { HipP } & \text { Hip pressure sequence } \\ \text { HipW } & \text { Hip width }\end{array}$


$I M G$

$I M G_{d e c}$

$I M G_{\text {inc }}$

$I M G_{R E G}$

$I M G_{\text {sit }}$

K

$L$

LHandP

LHdom

$n$

$N$

Ovlp

$R E G$

$R E G_{C P, y}$

RHandP

RHdom

$s_{k}$

$T$

T1

$\mathrm{T} 2$

$T d_{\text {opt }}$

$T h$

$v_{0}, v_{1}$
Region index

Pressure image

Image composed of pixels found to decrease

Image composed of pixels found to increase

Image which contains all regions before hand region verification

Average image in which the body sits upright

Gain

Wavelet level of decomposition

Left hand pressure sequence

Left hand dominant pressure

Digital time sample index

Number of digital time samples in the sequence

Allowable overlap between the hand and hip regions

Region detected as a possible hand

Regional center of pressure location

Right hand pressure sequence

Right hand dominant pressure

Output of sensor $k$

Time constant

Time from the start of the sit-to-stand transfer to the seat-departure

Time from the seat-departure of the sit-to-stand transfer to stability

Optimal denoising threshold

Signal threshold defining the stable part of the transfer response

Coefficients of the linear approximation

xix 


$\begin{array}{ll}V_{o p t} & \text { Vector of the optimal coefficients in the linear approximation } \\ v t h r & \text { Threshold for sagittal deviation classification } \\ x_{k} & \text { x-coordinate of the location of sensor } k \\ y_{e s t} & \text { Estimated model response to an input step in the time domain } \\ Y_{e s t} & \text { Estimated model response to an input step in the Laplace domain } \\ y_{k} & \text { y-coordinate of the location of sensor } k \\ \sigma_{y_{d t r}} & \text { Sagittal deviation of the detrended trajectory of the CP y-coordinate } \\ \omega_{n} & \text { Natural frequency } \\ \zeta & \text { Damping ratio } \\ \tau & \text { Time delay }\end{array}$




\section{Chapter 1:}

\section{Introduction}

\subsection{Thesis Motivation}

Canada is facing a fundamental shift in its demographics with an unprecedented growth in its senior population. Health Canada estimates that by 2026 , one in five Canadians will have reached the age of 65 , representing $20 \%$ of the population [1]. As the members of this population age, their needs for costly healthcare monitoring increase. Many hospitals and nursing care facilities across the country are already reaching their financial limits and the expected workloads are exceeding the personnel available. There is therefore a growing demand for technology that could aid in the care of seniors, whether in health institutions or in their own homes. The biomedical engineering community, along with organizations such as Health Canada and various academic institutions, is attempting to respond to this need by developing smart home technologies that could provide automated monitoring of the health and well-being of seniors [2]-[4]. This could provide the senior person with a higher degree of independence and quality of life, while decreasing the financial burden on the healthcare system. 


\subsection{Problem Definition}

Monitoring the functional mobility of seniors is key in the assessment of their physical health and overall well-being [5], [6]. One of the greatest challenges in smart home monitoring is that human motion and behaviour are not easy to predict or to interpret. Even once an event or pattern is successfully recognized, its meaning can be entirely different depending on the context within the environment. In this thesis, context is defined as relevant information which can provide additional insight to characterize the situation within the smart home. A schematic of a traditional monitoring system is shown in Figure 1.1, beginning with sensor data collection.

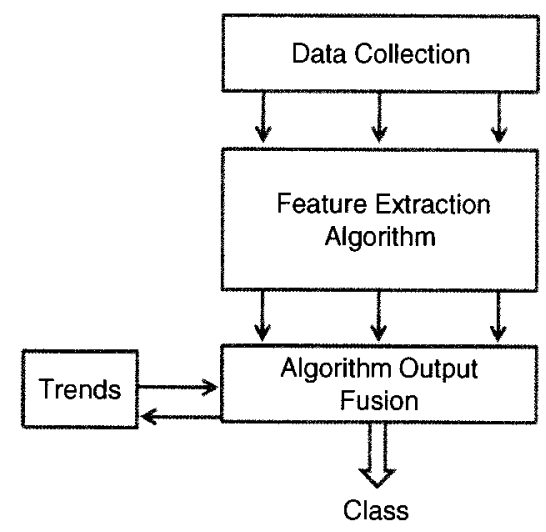

Figure 1.1: Traditional monitoring system.

The collected data is sent to a feature extraction algorithm which in turn produces several outputs. The algorithm output fusion then combines the information from these features with the trends observed over time to make a classification decision regarding the patient's status. 
This process can be greatly improved by a system that is capable of both detecting significant characteristics of the occupant and acquiring awareness of the context in which they were obtained. Should discrepancies occur between the patterns and the context, the system could review its processing and either modify its algorithm parameters or choose to extract alternate features.

\subsection{Thesis Objectives}

The main objective of this research is to provide unobtrusive monitoring of the functional mobility of an occupant within a smart home environment. The system should be aware of its context and adaptable to its environment. To accomplish this, pressure sensors are used to collect data throughout the home. Digital signal processing algorithms are then designed to extract significant clinical information about the occupant's mobility while performing basic tasks. The algorithms are tested using simulation as well as clinical trials.

\subsection{System Overview}

The system designed in this thesis proposes three main enhancements to the traditional monitoring system. In the clinical monitoring environment, it is often necessary to extract features from multiple perspectives to obtain the most representative information. The first enhancement in the thesis design, depicted in Figure 1.2, is to develop several different feature extraction algorithms to observe significant mobility features from different perspectives. 


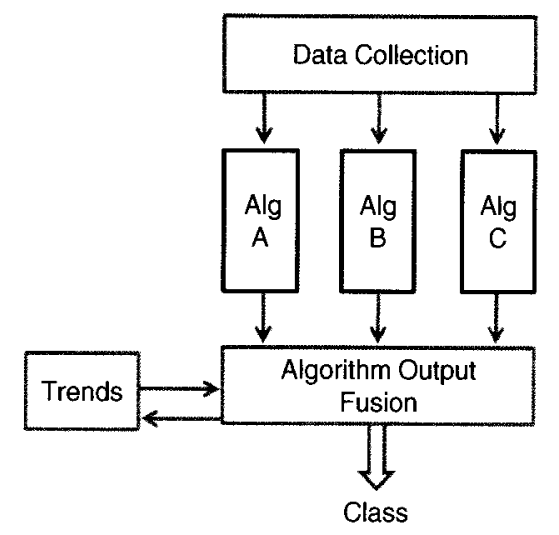

Figure 1.2: Monitoring system with three feature extraction algorithms to observe significant clinical features.

Depending on the situational context at any given time, some features may be more suitable than others in making the decision regarding the mobility status of an occupant. The second enhancement to the traditional system, depicted in Figure 1.3, is the introduction of context regarding the subject or their environment. This allows the selection of the appropriate algorithms to be applied, either by an automated decisionmaker or by a supervising physician. Context is also provided to the fusion level to verify the outputs obtained by the algorithms before a classification decision is made.

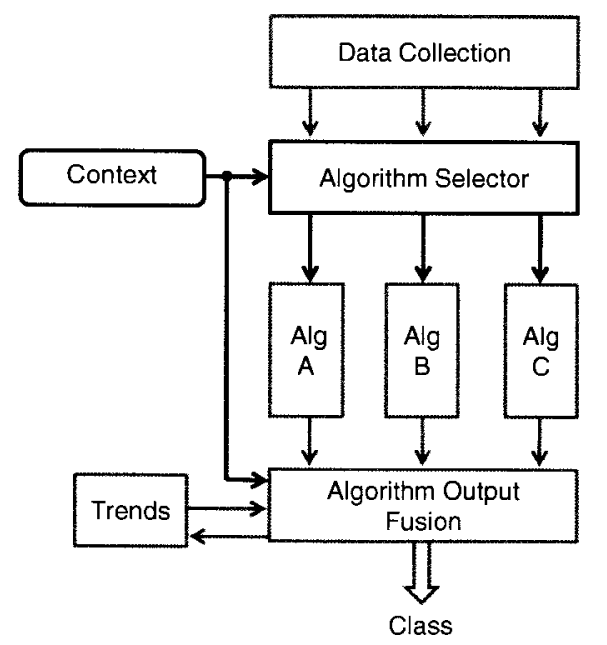

Figure 1.3: Monitoring system with introduction of context to select appropriate algorithms. 
The third enhancement is to incorporate a feedback loop from the algorithm output fusion to both the algorithm selector and the three algorithms themselves, as depicted in Figure 1.4. This allows for system verification, resulting in either modifying the algorithm parameters or selecting alternates based on the provided context. This process can be repeated until a satisfactory decision has been reached.

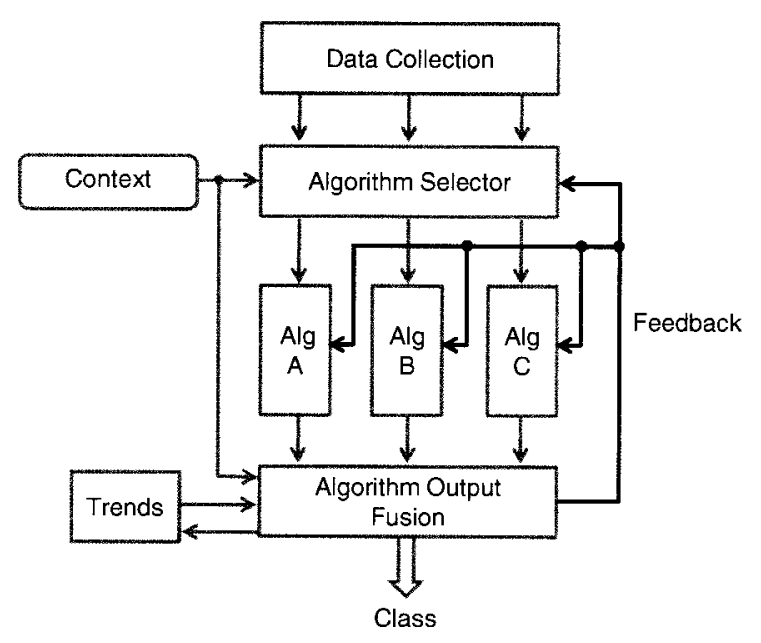

Figure 1.4: Monitoring system with introduction of feedback loop to provide context-aware feedback to selector and algorithms.

The three enhancements presented in this section allow for a design that is contextaware and adaptable to its environment.

\subsection{Research Methodology}

The system design in this thesis is intended to monitor a smart home occupant over time to detect changes in mobility and prevent the occurrence of a hazardous event. Full verification of the system would require the observation of trends from numerous patients over months of monitoring to determine whether or not mobility decline was successfully predicted. The collection of such data would take several years and would fall outside the 
scope of this thesis. Instead, it was decided to proceed with the thesis research in two stages. The first stage was to perform preliminary experiments with several seniors undergoing periods of rehabilitation after hip surgery to observe changes in mobility characteristics and to concretize the system design considerations. The second stage was to conduct controlled clinical trials with healthy participants as well as seniors who had recently suffered hip-fractures or strokes in order to simulate the mobility decline which can occur over extended periods of time due to underlying conditions. While these trials are not exhaustive clinical grade assessments, the collected data allows the design of clinical feature extraction algorithms and provides the preliminary verification of their results.

\subsection{Thesis Contributions}

This thesis includes several contributions to the field of smart home monitoring. Given the cross-disciplinary nature of this work, the publications include several authors from both the engineering and healthcare communities. Most of the publications include both of the co-supervisors of this thesis: Dr. Rafik Goubran, professor in the Department of Systems and Computer Engineering at Carleton University and Dr. Frank Knoefel, physician specializing in geriatric rehabilitation and vice-president of medical affairs and health information at Bruyère Continuing Care in Ottawa, ON, Canada. Many of the publications also include Dr. Heidi Sveistrup and Dr. Martin Bilodeau, professors in the School of Rehabilitation Sciences at the University of Ottawa, along with their students. Several publications involve collaboration with three members of the Digital Signal Processing lab in the Department of Systems and Computer Engineering at Carleton 
University: (1) Megan Holtzman (formerly M. H. Jones), a Ph.D. candidate who focuses on the analysis of breathing signals using pressure-sensitive mats, Idana Veledar, a Masters student who investigated sit-to-stand transfers using pressure sensing, and Christophe Herry, a post-doctoral fellow who worked on floor plate signals measured during sit-to-stand transfers.

This section outlines the main contributions, the thesis chapters in which they are presented and the resulting publications.

Contribution 1: A monitoring system for seniors was designed which integrates several different technologies into the smart home environment. This system is introduced in Chapter 2 and has been published in [7]:

A. Arcelus, M. H. Jones, R. Goubran, and F. Knoefel, "Integration of smart home technologies in a health monitoring system for the elderly," in Proc. $21^{\text {st }}$ Int. Conf. Advanced Inform. Networking and Applicat. Workshops, Niagara Falls, ON, Canada, 2007, pp. 820-825.

Contribution 2: The use of pressure sensing within the smart home is introduced in Chapter 2 and has been explored in various modalities throughout the thesis. A smart monitoring system was designed based on pressure sensors located throughout the home and has been published in [8]. Algorithms were designed to analyze breathing signals collected by pressure sensors and have been published in [9].

M. H. Jones, A. Arcelus, R. A. Goubran, and F. Knoefel, "A pressure sensitive home environment," in Proc. IEEE Int. Workshop Haptic Audio Visual Environments Applicat., Ottawa, ON, Canada, 2006, pp. 10-14.

M. Holtzman, A. Arcelus, R. Goubran, and F. Knoefel, "Breathing signal fusion in pressure sensor arrays," in Proc. IEEE Int. Workshop Medical Meas. and Applicat., Ottawa, ON, Canada, 2008, pp. 71-76. 
Contribution 3: The experimental setup and verification methods for the analysis of mobility using pressure sensors were designed in collaboration with a clinical team. They are presented in Chapter 3 and parts have been published in [10], [11] and [12].

D. Blaney, D. Cloutier, K. Irvine, K. Jensen, A. Arcelus, F. Knoefel, R. Goubran, H. Sveistrup, and M. Bilodeau, "Development of a checklist to differentiate stand-to-sit bed transfer patterns between healthy older adults and those who had a stroke or hip fracture," presented at the $30^{\text {th }}$ Annu. Scientific Meeting Canadian Geriatrics Soc., Ottawa, ON, Canada, 2010.

J. Medwid, O. Habib, A. Arcelus, M. Bilodeau, F. Knoefel, R. Goubran, and H. Sveistrup, "The Ottawa sit-to-stand checklist for task analysis," presented at the $30^{\text {th }}$ Annu. Scientific Meeting Canadian Geriatrics Soc., Ottawa, ON, Canada, 2010.

H. Sveistrup, M. Fitzpatrick, J. McLeod, O. Habib, A. Arcelus, M. Bilodeau, R. Goubran, and F. Knoefel, "Clinical populations can be distinguished on sit-to-stand movement parameters," to be presented at the ACRM ASNR Joint Educ. Conf. Progress Rehabil. Res., Montreal, QC, Canada, 2010.

Several algorithms were designed for the extraction of features and classification of mobility through the analysis of pressure sequences placed under the mattress and on the floor. They focus on the analysis of an occupant's weight transfers from a sitting to a standing position while exiting their bed. The main related contributions for the analysis of sit-to-stand (SiSt) transfers are listed in the following three sections.

Contribution 4: An algorithm was designed for the detection and characterization of regions of interest (ROI) from pressure image sequences. The algorithm focused on the differences between consecutive images to detect the ROI and allow the observation of the pressure sequences of each region over time. Significant features were extracted and presented in the form of an interactive clinical assessment tool. This allowed the visual detection of abnormal mobility cases as well as the automated classification of the 
occupant's SiSt symmetry. This ROI-based algorithm is described in Chapter 4 and has been published in [13]:

A. Arcelus, I. Veledar, R. Goubran, F. Knoefel, H. Sveistrup, and M. Bilodeau, "Measurements of sit-to-stand timing and symmetry from bed pressure sensors," to appear in IEEE Trans. Instrum. Meas. May 2011.

Work on manually defined ROI which could be used in timing measurements was published in [14]:

I. Veledar, A. Arcelus, R. Goubran, F. Knoefel, H. Sveistrup, and M. Bilodeau, "Sit-tostand timing measurements using pressure sensitive technology," in Proc. IEEE Int. Instrum. Meas. Technology Conf., Austin, TX, USA, 2010, pp. 1337-1340.

Contribution 5: The second algorithm was designed to analyze the center of pressure (CP) trajectory of the occupant in the sagittal plane (vertical plane which divides the body into left and right halves) during bed-exit. It provided clinical feature extraction from the trajectory as well as automated impairment classification based on sagittal deviation. Significant differences were found between the features of healthy and mobility-impaired SiSt transfers, and they behaved similarly to those extracted from more obtrusive measures published in recent literature. This CP-based algorithm is presented in Chapter 5 and has been submitted for review in [15]:

A. Arcelus, R. Goubran, H. Sveistrup, M. Bilodeau and F. Knoefel, "Context-aware smart home monitoring through pressure measurement sequences," submitted to IEEE Trans. Instrum. Meas. Jul. 2010.

Contribution 6: The third algorithm was designed to measure the duration of the SiSt transfer using pressure sensors under the bed as well as on the floor. The start of the transfer was automatically detected based on the CP motion on the bed and the end was 
automatically detected by the stabilization of the floor pressure sequences. The duration results compared well to those obtained from clinical video analysis as well as to those found in related publications. This duration-based algorithm is described in Chapter 6 and has been published in [16]:

A. Arcelus, C. L. Herry, R. A. Goubran, F. Knoefel, H. Sveistrup, and M. Bilodeau, "Determination of sit-to-stand transfer duration using bed and floor pressure sequences," IEEE Trans. Biomed. Eng., vol. 56, no. 10, pp. 2485-2492, Oct. 2009.

Additional analyses performed with the duration-based algorithm along with related implications for the clinical field were published in [17] and [18]:

F. Knoefel, A. Arcelus, R. Goubran, M. Bilodeau, and H. Sveistrup, "Can a mat sensor algorithm identify mobility challenges in older adults getting out of bed?," in Proc. $7^{\text {th }}$ World Conf. Int. Soc. for Gerontechnology, Vancouver, BC, Canada, 2010, pp. 225.

S. Mondoux, P. Carlson, N. Foubert, A. Arcelus, F. Knoefel, R. Goubran, M. Bilodeau, and H. Sveistrup, "Smart mat technology: can it differentiate bed transfers in older healthy and older hip fracture patients?," presented at the Annu. General Meeting Regional Geriatrics Program, Ottawa, ON, Canada, 2009.

Several algorithms were designed for the introduction of context into the monitoring system design. The main related contributions are listed in the following three sections.

Contribution 7: An algorithm was designed to extract clinical features from pressuresensitive grab bars. The algorithm analyzed the pressure sequences recorded during transfers on and off of a commode. These results could either validate or contradict the analysis of SiSt transfers recorded from the bed pressure sequences, providing sensed context for mobility classification. The grab bar algorithm is presented in Chapter 7 and its various algorithm components were published in [19] and [20]: 
A. Arcelus, M. Holtzman, I. Veledar, R. Goubran, H. Sveistrup, and P. Guitard, "Contact location estimation from a nonlinear array of pressure sensors," in Proc. IEEE Int. Instrum. Meas. Technology Conf., Victoria, BC, Canada, 2008, pp. 1969-1973.

M. Holtzman, A. Arcelus, I. Veledar, R. Goubran, H. Sveistrup, and P. Guitard, "Force estimation with a non-uniform pressure sensor array," in Proc. IEEE Int. Instrum. Meas. Technology Conf., Victoria, BC, Canada, 2008, pp. 1974-1979.

The results of the grab bar feature extraction with data collected from young and senior adults were published in [21]:

A. Arcelus, M. Holtzman, R. Goubran, H. Sveistrup, P. Guitard, and F. Knoefel, "Analysis of commode grab bar usage for the monitoring of older adults in the smart home environment," in Proc. $31^{\text {st }}$ Int. Conf. IEEE Eng. Med. Biol. Soc., Minneapolis, MN, USA, 2009, pp. 6155-6158.

Related implications for the clinical field were published in [22]:

M. Kennedy, A. Arcelus, P. Guitard, R. Goubran, F. Knoefel, and H. Sveistrup, "Using a commode changes how older adults, adults post-stroke and adults post-hip-fracture sit and stand from a toilet," presented at the $30^{\text {th }}$ Annu. Scientific Meeting Canadian Geriatrics Soc., Ottawa, ON, Canada, 2010.

Contribution 8: A simulated context-aware environment was designed to demonstrate the function of the smart home monitoring system of this thesis. Scenarios were simulated using the algorithm results collected from participants during actual clinical trials. The function of the proposed design is presented in Chapter 8 and has been published in [23]:

A. Arcelus, R. Goubran, F. Knoefel, H. Sveistrup, and M. Bilodeau, "Context-aware smart home monitoring through pressure measurement sequences," Proc. IEEE Int. Workshop Medical Meas. and Applicat., Ottawa, ON, Canada, 2010, pp. 32-37. 


\subsection{Document Layout}

This thesis is divided into nine chapters beginning with a background review in Chapter 2. Chapter 3 describes the experimental setup and clinical data collection. Chapters 4,5 and 6 contain the three algorithms designed for the monitoring system, as introduced in the system overview of this chapter. Chapter 7 introduces the sources of context in the system design and Chapter 8 incorporates context-awareness into smart home monitoring through a simulated environment. Chapter 9 presents the thesis conclusions and suggestions for future work. 


\section{Chapter 2:}

\section{Background Review}

\subsection{Introduction}

This chapter reviews the background information relevant to the thesis in three main sections. The first, Section 2.2, introduces the forms of context-awareness currently used in both general purpose and health-focused smart homes. Section 2.3 then describes the SiSt transfer and the methods currently used for its assessment. Finally, Section 2.4 introduces pressure sensing as the main form of data collection throughout this thesis. The chapter ends with conclusions in Section 2.5.

\subsection{Context-Awareness in the Smart Home Environment}

Smart home technology is a rapidly growing field as its development and applications become increasingly relevant to both the engineering and healthcare communities [24], [25]. This section presents recent work published on the incorporation of contextawareness into general purpose smart homes as well as into health-focused smart homes. 


\subsubsection{General Purpose Smart Homes}

Recent work in context-aware smart home monitoring is largely focused on providing context through the localization of the occupant, both throughout the home and with respect to their surroundings.

In [26], Lu et al. present an activity recognition system in a single-occupant smart home through various embedded analog sensors and a wireless sensor network. Their primary focus was using location information to incorporate context-awareness into the activity recognition. To accomplish this, they included piezoelectric floor blocks which tracked the motion of the occupant throughout the home.

Kim et al. [27] installed an array of pyroelectric infrared sensors on the ceiling to continuously locate a resident within their smart home. Using a Bayesian classifier, the resident's location was recognized and transmitted to the smart home server with the future goal of providing location-based intelligent services such as lighting and temperature control.

In [28], Brdiczka et al. used cameras, microphone arrays and headset microphones to track occupants within a multi-user smart environment. Video tracking, noise detection and speech recognition were implemented to analyze the distances from their bodies and others as well as from objects in the environment such as tables or chairs. This information was then used to determine the activity being performed.

\subsubsection{Health Monitoring Smart Homes}

Smart homes specifically geared towards patient health monitoring have also used localization as a primary source of context-awareness. 
In [29], Pung et al. presented middleware for pervasive senior care whose framework included a context data management layer. This layer defined physical spaces to include context-awareness into their monitoring system. Their spaces were organized into either 'person', 'home', 'clinic' or 'hospital' to allow efficient processing of queries for further data acquisition.

In [30], Chiti et al. presented a wireless communication system for context-aware health monitoring that combined mobile body sensor networks with independent wireless sensor networks embedded in the environment. The target localization was used both to provide context-awareness and to manage sleep/active states of its sensors with the goal of increasing energy efficiency.

\subsubsection{TAFETA Smart Home}

Carleton University is involved in a joint Ontario-based project entitled Technology Assisted Friendly Environment for the Third Age (TAFETA) ${ }^{1}$. The co-lead in this project is Bruyère Continuing Care, one of Canada's largest providers of continuing care for seniors, rehabilitation, palliative care, and primary care [31]. The project's other members include additional healthcare institutions, schools, and industry partners in Ontario, Canada. Its primary mission is to identify and develop technology that will provide a safe living environment and be responsive to the health needs of the senior population [7]. The TAFETA Smart Home provides the framework and laboratory for the research subject to this thesis.

\footnotetext{
${ }^{1}$ A significant portion of the contents in this section has also been published in [7].
} 


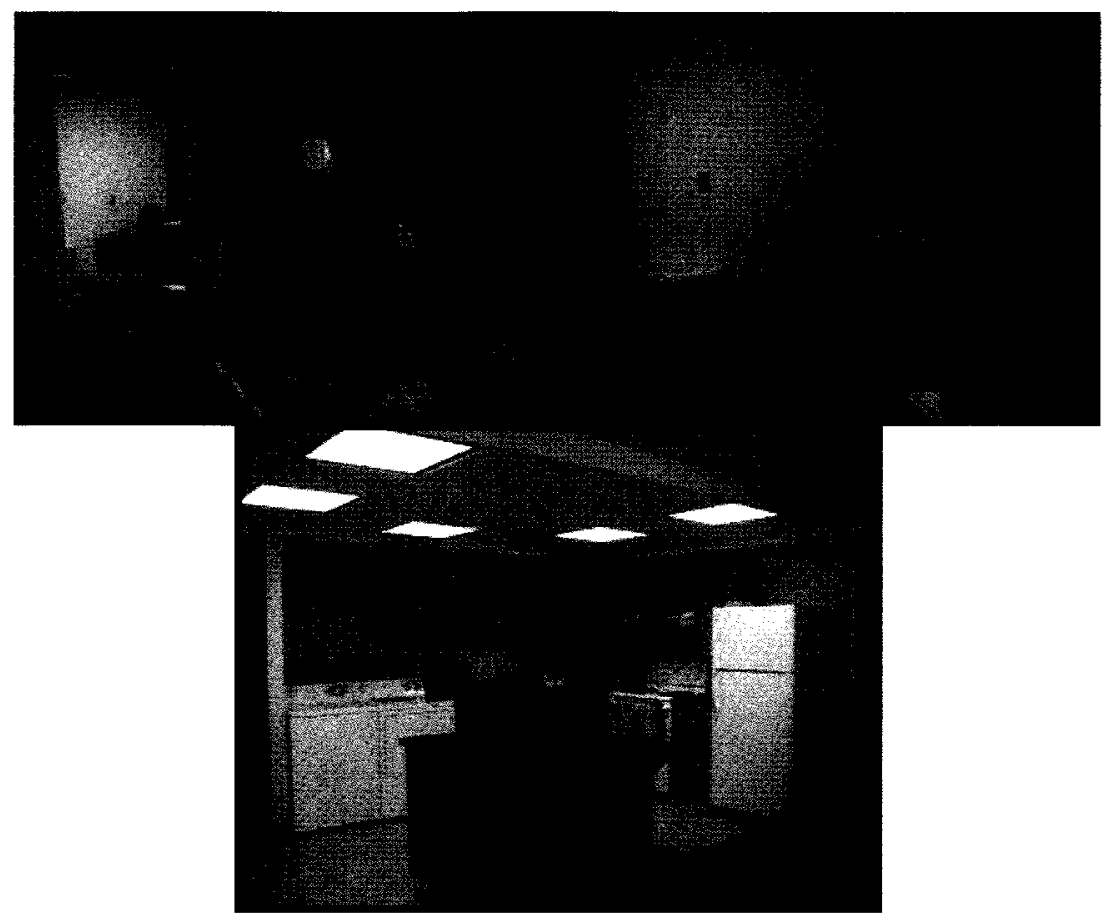

Figure 2.1: Views of the bedroom, lavatory and kitchen in the TAFETA 'Independent Living Apartment' laboratory, located in the Élisabeth Bruyère Hospital in Ottawa, ON, Canada.

The TAFETA prototype 'Independent Living Apartment', shown in Figure 2.1, is located in the Élisabeth Bruyère Hospital (ÉBH) of Bruyère Continuing Care in Ottawa, ON, Canada. Various types of sensing technologies are presently under investigation as part of the smart home concept [7]. Several of these have been incorporated into the prototype apartment, such as magnetic switches, motion sensors and pressure-sensitive mats. Others are undergoing experimentation in the Digital Signal Processing lab at Carleton University, such as microphone arrays and electronic noses, as well as in the School of Rehabilitation Sciences at the University of Ottawa, such as pressure-sensitive grab bars. As introduced in Chapter 1, this thesis focuses on the monitoring of the functional mobility level of an occupant within the smart home. The incorporation of 
context-awareness within the smart home becomes a major goal of the system design and will be further explored in later chapters of this thesis.

\subsection{Smart Sit-to-Stand Transfer Analysis}

The transfer of a person's body weight from sitting to standing requires an adequate level of strength, coordination and balance [32], [33]. The SiSt transfer is widely used in the assessment of functional mobility in seniors [34]. Since bed-exit is a task performed at least once every day by an independently mobile individual, its analysis could become part of an ongoing monitoring system. Early detection of a decreasing ability to transfer could help in the prevention of falls, currently a significant public health concern for the senior population [34].

In clinical settings, tests involving in-vivo measurements of the SiSt, such as the Berg Balance Scale [36], are performed as part of an overall assessment of physical condition or to verify the progress of rehabilitation over time. The increasing needs of the senior population and the incorporation of these tests into the smart home environment are driving the need for automated methods of transfer assessments. Following is a review of recent work published in the area of smart SiSt transfer analysis.

\subsubsection{Image Processing}

Techniques for analyzing SiSt video sequences have been recently introduced using image processing in the Gauss-Laguerre domain [37]. Natural markers were identified on the body and their trajectories were obtained throughout the SiSt sequence. A human body model was then run to obtain angles for the hip and ankle joints along with angular 
velocities and durations in order to compare differences between young and senior subjects. Image analysis has shown to be valuable in remote assessments of transfers as well. In Durfee et al. [38], videos were captured of remote subjects performing various SiSt tests. Image processing was then performed on the video images to measure the angles of joints used in the transition, such as shoulder abduction, shoulder rotation and knee flexion.

Bed images were obtained by Veledar [39] from SiSt sequences using pressure sensors. Four methods were used to identify regions of interest from individual image frames: manual definition, binary cluster detection, template matching and edge detection. Major challenges included low-resolution images, high noise levels, and sporadic movements by clinical participants. Future recommendations included the investigation of noise filtering methods and the carrying of information between consecutive frames of the transfer.

\subsubsection{Center of Pressure Trajectories}

The measurement of balance and mobility in a SiSt transfer often involves several components of equipment worn on the body to track the center of mass $(\mathrm{CM})$ or center of pressure (CP) over time. Riley et al. [40] investigated the differences in CM between successful and failed SiSt attempts using a four-camera kinematic data acquisition system with arrays of infrared light emitting diodes (LEDs) attached to each of 11 segments of the body. In addition to wearable sensors, CP information can be simultaneously tracked using force plates under the feet or buttocks. Mathiyakom et al. [41] used force plates to track the mechanical demands across the lower body and Scholz et al. [42] used them to 
examine the variability of CP displacement in both medio-lateral and anterior-posterior directions. Lomaglio et al. [43] used force plate data from each foot to observe weightbearing symmetry in the SiSt transfers of individuals post-stroke.

In recently published work the pressure exerted by the body on the seat prior to seatdeparture has become a feature of interest in distinguishing levels of mobility. Anan et al. [44] studied the effect of chair cushion thickness on the SiSt motion of seniors. To accomplish this they used a force plate under the chair to observe the CP of the buttocks throughout the transfer in addition to the typical marker position system on the body with a force plate under the feet. Duclos et al. [45] compared the displacement and stability of the $\mathrm{CP}$ in the SiSt transfers of healthy volunteers to those of hemiparetic patients with different initial foot placements. In addition to infrared markers on the body and force plates embedded in the floor under each foot, they included a laboratory chair instrumented with strain gauge transducers to record the CP beginning from the seated position.

\subsubsection{Duration Measurements}

Successful measurements of SiSt duration have recently been made using accelerometers attached to the trunk of the body. Janssen et al. [32] compared transfer durations of healthy subjects to patients with stroke at different phases of recovery. Bidargaddi et al. [46] developed a wavelet-based algorithm for detecting and calculating the durations of transfers from both young and senior adults.

Accelerometers have been combined with gyroscopes to detect and measure SiSt transfers in Parkinson's patients [47] as well as to distinguish between high and low-fall- 
risk seniors [48], [49]. Others have used several high-speed cameras and reflective markers or arrays of LEDs placed on the joints of the subject to record the angles of each body segment (trunk, legs, head, etc.) during the transfer. In addition to the markers, one or several force plates are often included under the feet to record the forces exerted on the ground by the subject's body, [50].

Duration measurements of the SiSt transfer were made by Veledar [39] on control patients using pressure sensors placed under a bed mattress. The duration of transfers were measured from the initial sitting position to the point of seat-departure by extracting a pressure sequence over time and applying thresholds to determine the starting point of the entire transfer as well as various intermediary phases.

\subsection{Pressure Sensing Within the Smart Home}

While many of the methods in Section 2.3 have shown promise in the assessment of SiSt transfers, their reliance on wearable sensors or clinical observation make them largely impractical for use within the smart home environment. Conversely, pressure sensing has shown itself to be a valuable form of smart occupant monitoring due to its unobtrusive and reliable nature. Pressure sensors have been used in the flooring of smart homes primarily for localization of the occupant [51]. It was shown in Section 2.3 that they have also been used in a few instances of SiSt transfer analysis. In the past, the TAFETA smart home has successfully incorporated pressure sensing into a bed to monitor an occupant's posture while lying down [52], as well as to extract respiratory rates, restlessness and movement [9], [53], [54]. In addition, a design was presented with sensors placed in 
furniture throughout the smart home to monitor presence and sitting posture, and into the flooring to monitor motion and gait analysis [8].

In this thesis, pressure sensing is used as the primary method of data collection in the smart home environment. Pressure sensors are embedded in common household items in both the bedroom and lavatory as part of a context-aware system that assesses the mobility of the occupant based on their weight transfers.

\subsection{Conclusions}

The rise of the senior population has led to valuable research and increasing interest in the field of smart home technology. This thesis proposes a context-aware smart home system to monitor the functional mobility of an occupant within the home. The variability in human behaviour and situational conditioning has further highlighted the need for context-awareness within a smart monitoring system. Recent work has been largely focused on localization as the main source of contextual information. In contrast, this thesis incorporates context through mobility-based features extracted from additional sensors throughout the home.

Early detection of a decrease in mobility could lead to the prevention of falls and fractures, common and hazardous occurrences amongst the senior population. The SiSt transfer is widely used as an assessment of functional mobility and therefore its analysis becomes a key focus of the monitoring system. While different methods have shown promise in collecting data during the SiSt transfer, they are largely impractical for use within the smart home environment. Recent mobility studies have successfully performed smart SiSt analysis using in-vivo clinical testing, video analysis, and sensors such as 
accelerometers and gyroscopes attached to the body. This thesis incorporates data collection through unobtrusive pressure sensing from underneath a bed mattress and on the floor, making it suitable for the ongoing monitoring performed within a smart home environment. 


\section{Chapter 3:}

\section{Experimental Setup and Clinical Data Collection}

\subsection{Introduction}

This chapter describes the setup and data collection for the three clinical experiments performed throughout this thesis. It begins in Section 3.2 with a description of the pressure sensing technology. Section 3.3 presents preliminary experiments performed in the Élisabeth Bruyère Hospital (ÉBH) to observe trends in SiSt transfers in patients undergoing rehabilitation after hip fractures. Section 3.4 describes the instrumentation and data collection performed during controlled clinical trials with the bed and floor sensor arrays to observe characteristics in participants of varying mobility levels. Section 3.5 ends the chapter with a description of the instrumentation and data collection performed with pressure-sensitive grab bars to provide a source of sensed context to the monitoring system. Figure 3.1 depicts the focus of this chapter within the monitoring system design. 


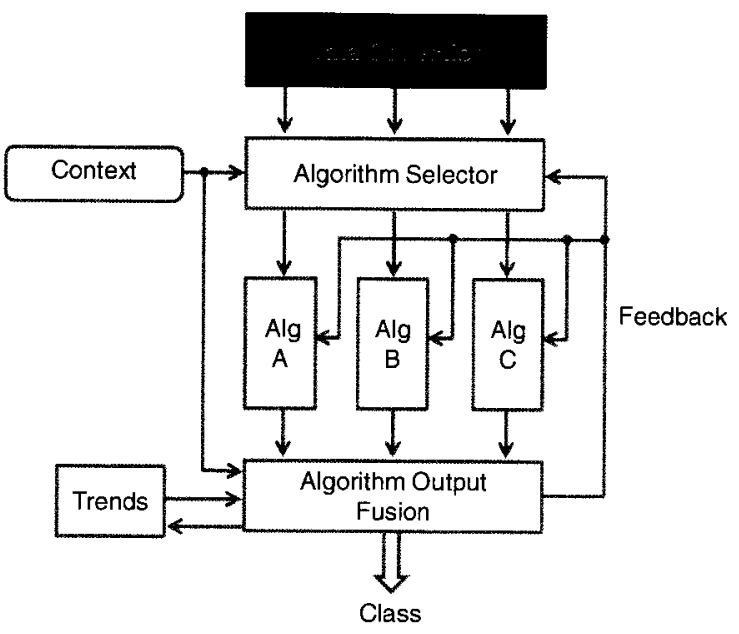

Figure 3.1: Monitoring system data collection.

\subsection{Kinotex® Sensor Technology}

All pressure sensing implemented throughout this thesis was composed of Kinotex ${ }^{\circledR}$ sensors manufactured by Tactex Controls Inc. They have a circular sensing surface with a diameter of $1 \mathrm{~cm}$. They are manufactured as either Single Point sensors, as shown in Figure 3.2(a), or as part of an array embedded into sensing surfaces such as mats and floor plates.

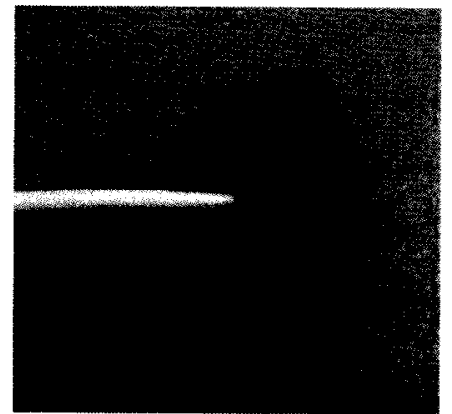

(a)

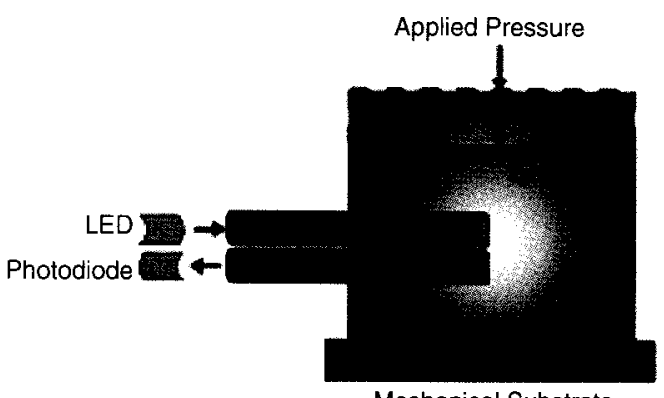

Mechanical Substrate

(b)

Figure 3.2: Kinotex@ pressure sensor (a) top view of a Single Point sensor and (b) side view schematic of the sensor components. 
Each sensor consists of two plastic optical fibres embedded into a foam cell. Light from an LED shines through the transmitting fibre to the cell. A pressure applied to the sensor foam causes a change in the properties inside the cell. The light is backscattered through the receiving fibre to the photodiode which produces a relative pressure output.

\subsection{Rehabilitation Experiments}

As introduced in Chapter 1, the goal of the design in this thesis is towards smart home monitoring of an occupant over time to detect changes in mobility before the occurrence of a hazardous fall. To observe the trends of SiSt transfers over time, preliminary experiments were performed throughout the course of a period of rehabilitation. For this purpose, six Bed Occupancy Sensors were used which contained 24 Kinotex ${ }^{\circledR}$ pressure sensors each. They were installed under the mattress of a bed in the rehabilitation ward of the ÉBH. The hospital provided a supervised setting for testing of the instruments over a period of time, approximating a smart home environment. Data was collected unobtrusively from patients who had undergone hip surgery, from the initial day of rehabilitation until they were released from the hospital to complete the recovery period in their own homes.

\subsubsection{Expected Rehabilitation Behaviour}

During the period of rehabilitation after surgery, it was expected that the SiSt transfer of a patient would become easier over time [55]. The time it took for the patient to exit the bed was expected to decrease as they became stronger and their level of mobility 
increased. Figure 3.3 displays the expected progression of the bed-exits over time, from the day of hip surgery to the day they would be released from the hospital.

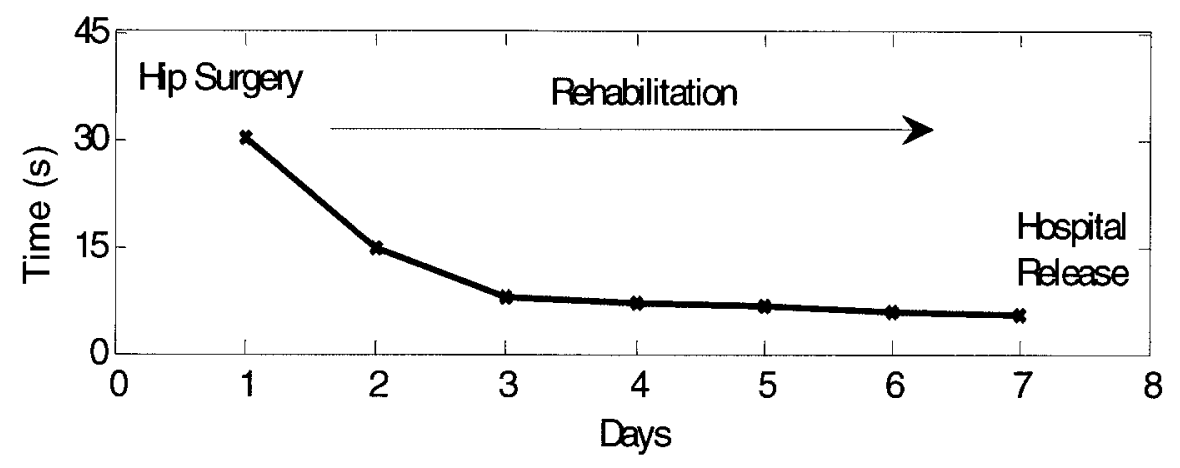

Figure 3.3: Expected bed-exit times for a rehabilitation patient.

\subsubsection{Actual Rehabilitation Behaviour}

The actual behaviour of a rehabilitation patient whose bed-exit times were recorded for five days is shown in Figure 3.4. As expected, the overall trend was to decrease over time. There was an exception on the third day, omitted from the figure to allow observation of the other values, when there was an unusually high output of $52 \mathrm{~s}$. It is expected that on this day the patient may have transferred from lying to sitting, then proceeded with some activity in the sitting position such as fixing their clothing before finalizing the bed-exit motion.

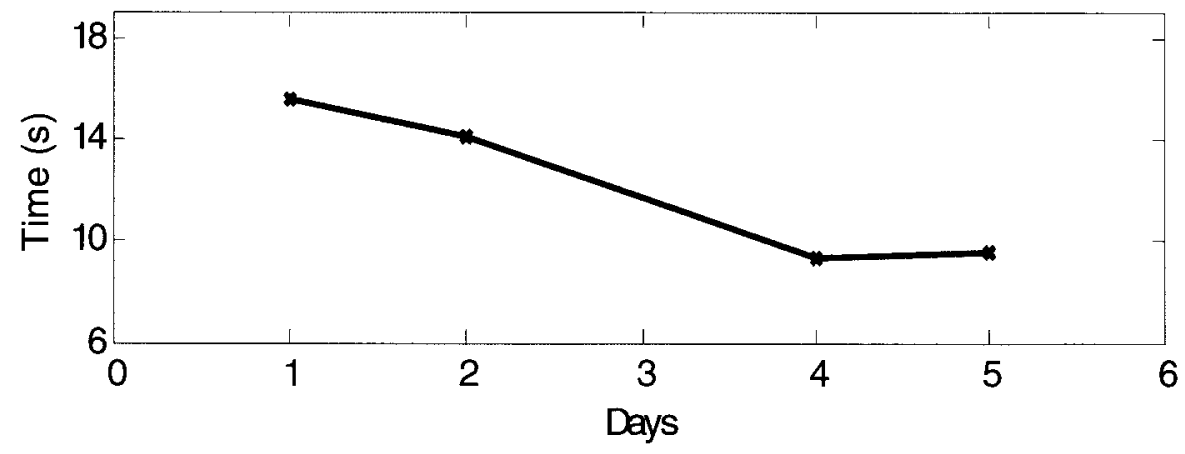

Figure 3.4: Actual bed-exit times for rehabilitation patient \#1. 
The results of a second patient are shown in Figure 3.5 over nine days of rehabilitation. The bed-exit times were considerably higher than the previous patient, starting at around $70 \mathrm{~s}$, as the patient had a more severe mobility impairment and likely paused several times throughout the transfer. Over time this patient displayed a decreasing trend, however it was accompanied by a higher degree of variability.

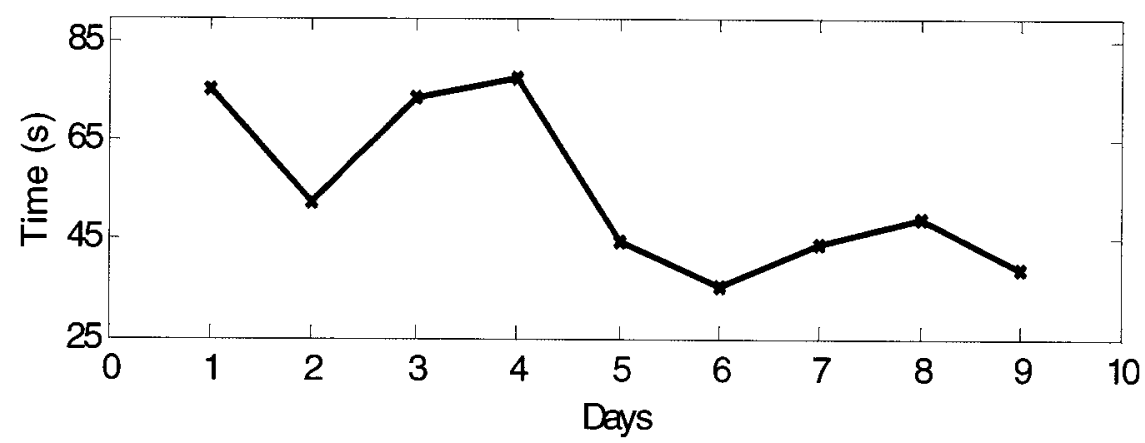

Figure 3.5: Actual bed-exit times for rehabilitation patient \#2.

\subsubsection{System Design Considerations}

The results of the rehabilitation tests highlighted several important points for the smart monitoring of a patient over time. The outlier of patient \#1 and the increased variability of patient \#2 indicated the importance of including context-awareness in the system design, since this could provide valuable insight into the environment surrounding the bed-exit analysis. The tests also highlighted the fact that an individual may perform the transfer in different steps or intervals of time, including pauses between lying to sitting and sitting to standing positions. An interval within the bed-exit, such as the sit-to-stand portion, was important to define such that the monitoring would focus on the same event over consecutive days. It also became evident that additional information would be required to analyze the patient's mobility throughout the rehabilitation period. To this 
end, feature extraction algorithms which could characterize the use of the hands during the bed-exit as well as the overall motion of the body on the bed would be of interest. These design considerations are explored further in later chapters of this thesis.

\subsection{Pressure Sensor Arrays}

\subsubsection{Equipment Setup}

\section{Pressure-Sensitive Bed Mats}

The bed sensing equipment was implemented using three $66 \mathrm{~cm}$ x $71 \mathrm{~cm}$ Tactex Large Sensor Arrays. Each array contains 44 pressure sensors embedded in a flexible mat as shown in Figure 3.6.

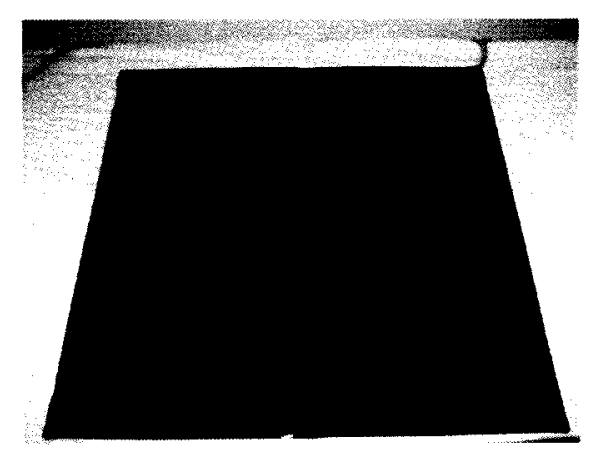

Figure 3.6: Large Sensor Array by Tactex Controls Inc.

The three mats were placed side-by-side underneath the mattress of a $210-\mathrm{cm}$ hospital bed, as shown in Figure 3.7(a), providing a total of 132 sensors. The mats were connected through an NPort server box into a Dell Latitude laptop computer. All sensor data were collected at a rate of $10 \mathrm{~Hz}$ through a Tactex software interface, and mapped to an array of corresponding values and locations. The mats were calibrated by performing baseline tests to record the unloaded response of each sensor. During testing, the sensor output 
values were referenced to these baseline values to obtain the true pressure deviation measurements.

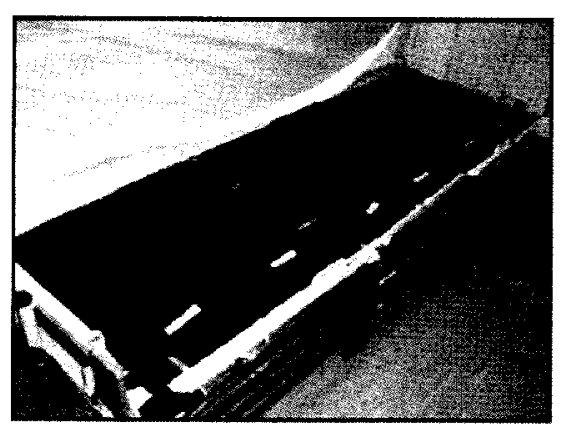

(a)

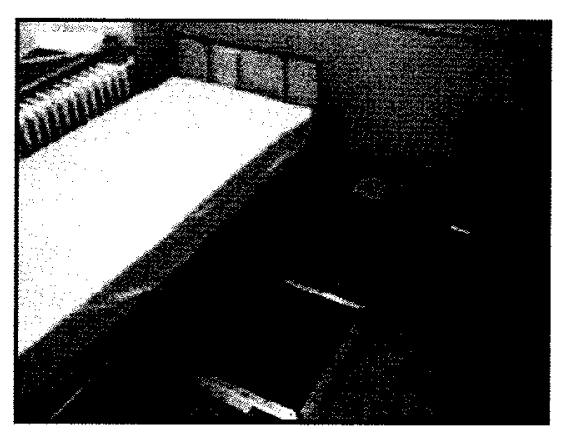

(b)

Figure 3.7: Pressure sensor arrays (a) pressure-sensitive bed mats and (b) pressure-sensitive floor plate.

\section{Pressure-Sensitive Floor Plate}

The Tactex floor plate consists of an array of pressure sensors embedded in a $60 \mathrm{~cm} \times 60$ $\mathrm{cm}$ floor plate. The sensing plate was placed on the floor directly beside the bed, as shown in Figure 3.7(b), such that the participant's feet would be completely within its boundaries during transfers on and off the bed. The raw outputs from the 2304 sensors were collected at a sampling rate of $50 \mathrm{~Hz}$. The floor plate was calibrated by a similar procedure to that of the bed mats, by performing baseline tests and referencing the sensor outputs to these values to obtain pressure deviation measurements.

\subsubsection{Clinical Experiments}

Twenty-five volunteers were recruited from both the Élisabeth Bruyère Research Institute (ÉBRI) and the ÉBH for the clinical trials. The participants were grouped into four categories:

1) Young healthy adults - ten individuals aged 18 to 39 years, from the ÉBRI staff; 
2) Healthy seniors - five individuals aged 65 years and above, from the volunteer office at the ÉBH;

3) Post-stroke seniors - five post-stroke individuals aged 65 years and above, from ÉBH rehabilitation;

4) Post-hip-fracture seniors - five post-hip-fracture individuals aged 65 years and above, from ÉBH rehabilitation.

All participants filled out medical history questionnaires and signed consent forms as approved by the Bruyère Continuing Care Research Ethics Board.

While the focus of the current project was on the SiSt transfer alone, data were collected continuously over several movements to obtain a more comprehensive assessment of the participant's mobility. Each subject was requested to perform consecutive transfers of lying supine on the bed, sitting upright, and then standing with both feet on the floor plate. After $10 \mathrm{~s}$, participants were asked to return from the standing to the supine lying position. Events were marked manually in the recording software during the data collection to denote the clear separation of each transfer. Figure 3.8 depicts the transfer sequence performed by all participants during data collection.

lying supine

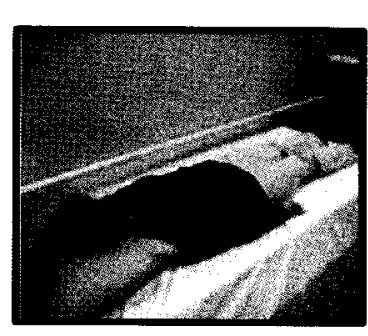

sitting upright

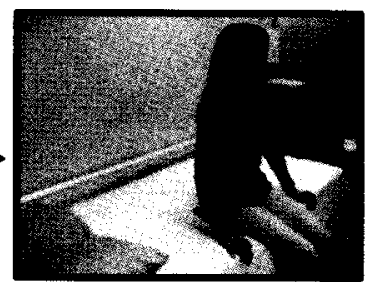

standing

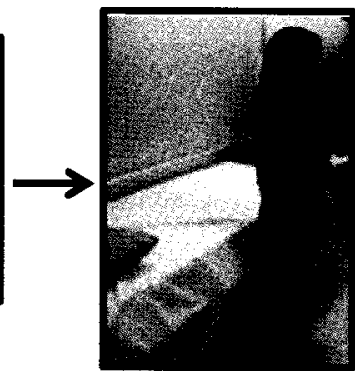

Figure 3.8: Transfer sequence from lying supine to standing performed by experiment participants during data collection. 


\subsubsection{Video Verification Protocol}

Two Sony high-definition cameras were mounted on tripod stands to provide front and side-view video data at 33 frames/s during all bed transfers. The goal of these two views was to provide the clinical gold standard for the analysis of the SiSt transfers. A database was created by the TAFETA clinical team with all of the significant features related to the assessment of postural transitions. The features characterized the body trunk motion, right and left arm usage, knee angles and foot placements. It also characterized key features for mobility assessment, such as transfer duration, symmetry and rocking. All videos were reviewed frame-by-frame by the clinical team and the characteristics of each transfer were documented in the database.

\subsection{Pressure-Sensitive Grab Bars}

\subsubsection{Grab Bar Instrumentation}

The instrumentation and testing of the pressure-sensitive grab bars were performed in the Daily Living Laboratory in the School of Rehabilitation Sciences at the University of Ottawa. Simulating a typical lavatory found in a private home, it was equipped with a standard sink, bathtub and toilet. Affixed to the toilet was a standard-sized commode with the dimensions shown in Figure 3.9.

Three pressure sensors were embedded in polyethylene foam and placed on each of the two commode grab bars. The sensors were equally spaced $6 \mathrm{~cm}$ apart such that a hand would activate at least one sensor during contact with the bar. Their placement began at 5 $\mathrm{cm}$ from the front end of each bar and covered the full length of the arm rests, with 
locations of $5 \mathrm{~cm}, 11 \mathrm{~cm}$ and $17 \mathrm{~cm}$. The bars were then wrapped in a semi-rigid plastic membrane to allow applied forces to be distributed over the neighbouring sensors.

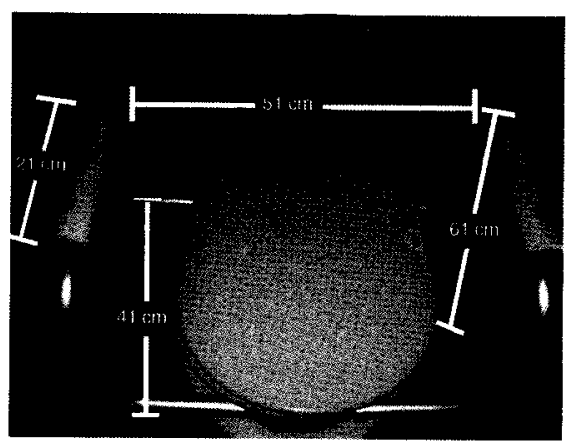

Figure 3.9: Commode grab bars instrumented with embedded pressure sensors.

Though individually each sensor measured the pressure exerted directly on it, the instrumented bars were treated as force sensors due to their small contact area compared to the spatial resolution over the length of the bar. The three sensors from each bar were interfaced with a desktop computer through a converter box and sampled at $10 \mathrm{~Hz}$. Finally, the entire set up and all associated wires were covered in a gray cloth to avoid any visual bias by the participants.

\subsubsection{Clinical Experiments}

Ten young adults aged 19 to 26 years and 11 seniors aged 65 to 80 years were recruited to participate in the grab bar study. The young adults were students or employees of the Ottawa Health Sciences Center and the seniors were recruited from organizations from the Ottawa area. All participants were in good overall health and were capable of executing transfers on and off the commode independently.

Participants attended a briefing where they were informed of the study objectives and signed the appropriate consent forms. They then proceeded to the lab where they were 
asked to begin standing with their back to the commode. They were instructed to use the grab bars to sit down on the commode, to remain seated without touching the bars for five seconds, to stand up using the grab bars and remain standing until instructed to repeat the sequence. This sequence was repeated five times with breaks in between when necessary. The pressure sensor outputs were recorded for the entire length of all trials and stored for subsequent analysis. 


\section{Chapter 4:}

\section{Region of Interest Detection and Characterization from Pressure Image Sequences}

\subsection{Introduction}

As introduced in Chapter 2, interest in pressure-based information collection is growing in the smart monitoring field due to its simple and unobtrusive nature. Though not one of the most common imaging modalities, pressure sensor arrays have recently been successfully used in medical imaging applications such as neck palpation for the detection of lymph nodes [56], in elasticity imaging of the breast for the detection of lesions [57], and in a transrectal probe for real-time imaging of the prostate [58]. This chapter converts pressure sensor measurements into sequences of images which allow the extraction of features to characterize mobility during SiSt transfers. An algorithm for automated region of interest (ROI) detection and feature extraction is designed based on sequential images constructed by a low-spatial-resolution array of pressure sensors ${ }^{2}$. Section 4.2 describes the image capture and pre-processing. The ROI detection algorithm

\footnotetext{
${ }^{2}$ A significant portion of the contents in this chapter has also been published in [13].
} 
is presented in Section 4.3 by combining the static analysis of single images with the dynamic analysis of image sequences over time. In Section 4.4 the regional pressure sequences are introduced and the related clinical features are defined. A prototype is presented of a clinical assessment tool created to aid in the assessment of SiSt transfers by clinicians along with an algorithm for the automated classification of symmetry. Results based on participant data are reported and verified in Section 4.5 , followed by a discussion in Section 4.6 and chapter conclusions in Section 4.7. Figure 4.1 depicts the focus of this chapter within the monitoring system design.

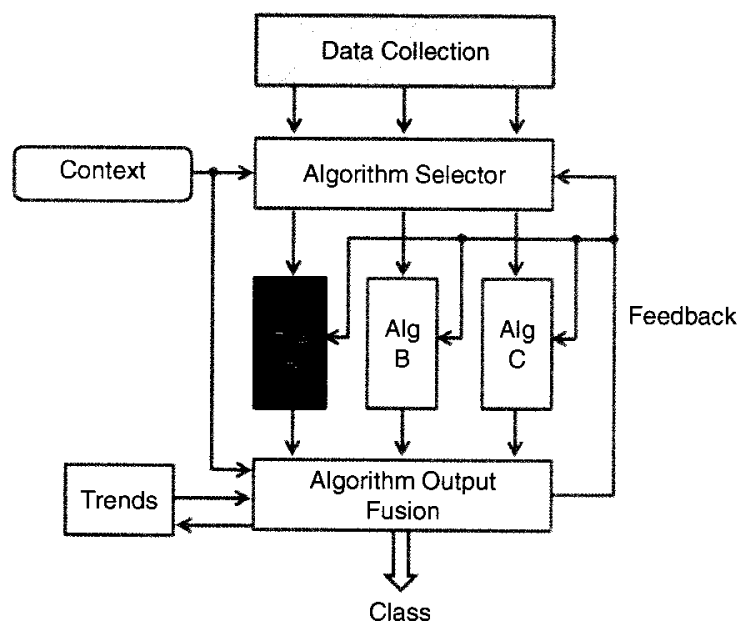

Figure 4.1: Monitoring system feature extraction algorithm A.

\subsection{Image Capture and Pre-Processing}

Image-registration techniques are often used to increase the available information extracted from medical images recorded by modalities such as Positron Emission Tomography (PET) and Computed Tomography (CT) [59], or by Functional Magnetic Resonance Imaging (FMRI) [60]. The registration techniques are based on two or more images taken from different viewpoints, by different sources, or at different times. In the 
imaging modality of this study there is only one sensor source which remains embedded in place and it is the object of interest which moves over the sensing space. The timevarying features become the main source of additional information sought from the recorded images. It is the change in pressure over sequential images that forms the basis of the automated algorithm.

Figure 4.2 displays three stages of an ideal image sequence during the SiSt transfer of a healthy participant. Figure 4.2(a) and Figure 4.2(b) depict a frame of 'sitting upright', as previously introduced in Section 3.4.2. This is followed by a point of transition where the participant uses both hands to push off the bed as seen in Figure 4.2(c) and Figure 4.2(d), and of the standing position once the participant has completed the transfer, as in Figure 4.2(e) and Figure 4.2(f). The image sequence is considered ideal in that the hips and hands appear as very distinct and identifiable regions.

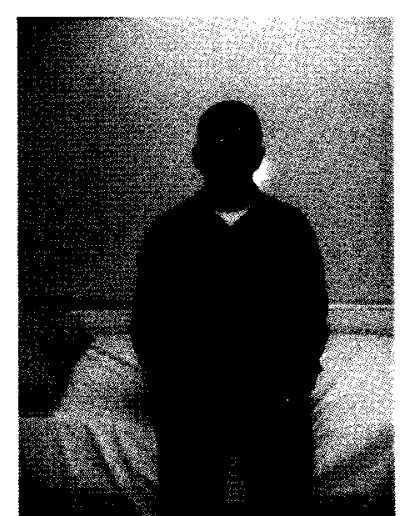

(a)

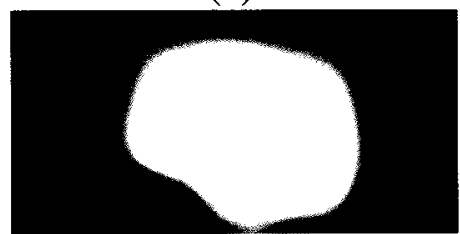

(b)

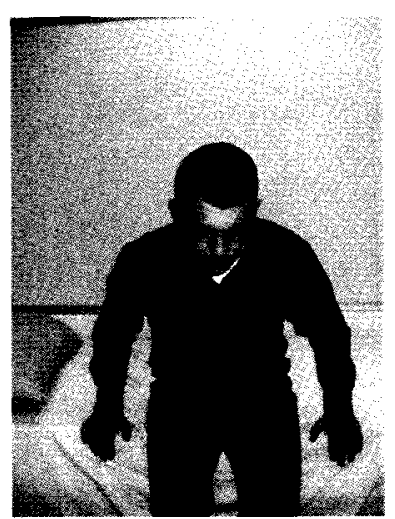

(c)

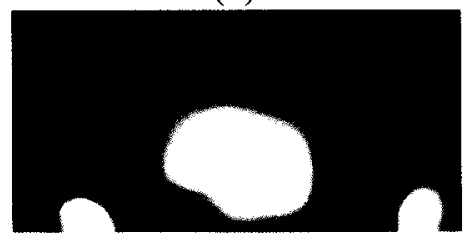

(d)

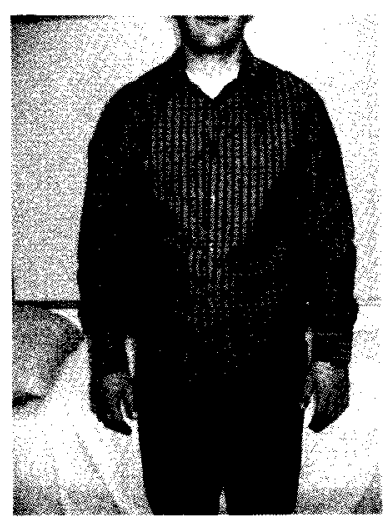

(e)

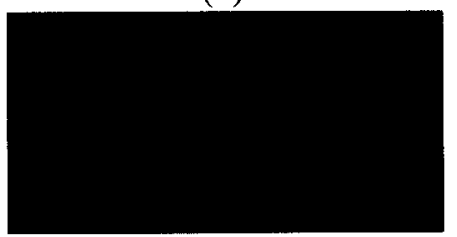

(f)

Figure 4.2: Ideal image sequence of SiSt transfer during bed-exit. 
At each frame $f$, the 132 sensor outputs were mapped to a pressure image $I M G(f)$ according to their locations in the mapping file provided by Tactex Controls Inc. The image was smoothed and resized so as to form the conjoined areas of pressure as well as the areas of void. Given that different bodies have different sizes and weights, the pressure images required normalization for each participant. Since a fully automated system was sought, this was done based on the closest approximation possible of the body weight on the bed, namely by the section of 'sitting upright'. The maximum and minimum pixel values of the first five frames were used to normalize the images to grayscale values between zero and one.

\subsection{Region of Interest Detection}

The ROIs which were the targets of the detection efforts were the hips and the two hands. Since the image sequences captured the transfer of the body from a sitting to a standing position, the regions were in motion throughout the transfer. The detection method would therefore need to combine the image information related to the physical dimensions of the body under consideration with the changes in the images as the body moved over time. Standard methods of edge detection [61] were first attempted such as the Laplacian of Gaussian [62] and the Canny [63] methods. These were unsuccessful in defining the borders of the regions due to the low spatial resolution of the pressure images and the amount of additional blurring required to establish the objects of interest. Attempts were also made with several forms of template matching [64], where an object of interest is approximated and correlated across the image to find a possible match. These were unsuccessful due to the variability in shape and position of each region as it moved 
rapidly throughout the sequence. An ROI detection and feature extraction algorithm was therefore designed for the clinical assessment of SiSt transfers. Its system architecture is depicted in Figure 4.3.

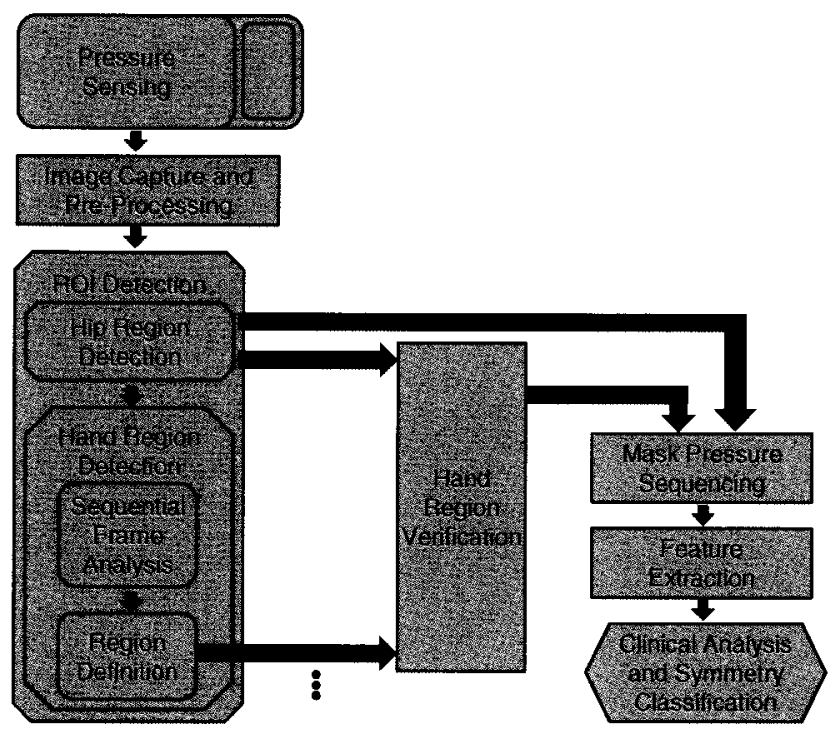

Figure 4.3: System architecture of the ROI detection and feature extraction from bed pressure image sequences.

\subsubsection{Hip Region Detection}

The start of the data segments containing each SiSt transfer were characterized by the body sitting upright on the bed for approximately five frames. During this time the pressure exerted on the mat was expected to be mostly contributed by the hip region and the remainder by the thighs. To obtain a representative picture of the contact region while the body sits upright, an image $I M G_{\text {sit }}$ was created by an average of the pixels in the first $F_{\text {sit }}=5$ images of the transfer segment as

$$
I M G_{\text {sit }}=\frac{\sum_{f=1}^{F_{s i t} I M G(f)}}{F_{\text {sit }}}
$$

An example of the grayscale $I M G_{\text {sit }}$ is found in Figure 4.4. 


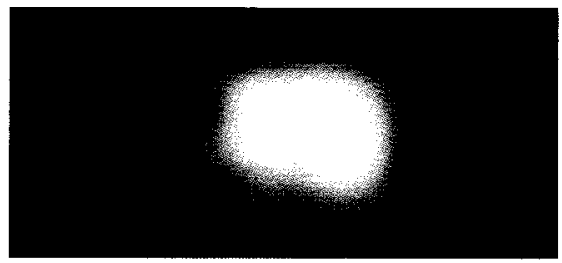

Figure 4.4: Image $I M G_{\text {sit }}$ of a healthy participant sitting upright.

The center of pressure of the seated region $C P_{s i t}, y$ was extracted to provide a frame of reference for the sitting position for subsequent processing. This was done by determining the CP of $I M G_{\text {sit }}$ based on its pixel locations and grayscale intensities.

Baseline calibration tests demonstrated that the average weight distribution of the body while sitting on the bed was $75 \%$ higher in the hip region, which carries the weight of the entire trunk, compared to that of the thighs which rest on the edge of the bed. A threshold of 0.75 was therefore defined on $I M G_{\text {sit }}$ with all values above the threshold set to one while all values below were set to zero. Binary cluster detection was performed to find objects that were connected by at least eight pixels. This left a binary shape of the hip region, which was stored as the transfer's binary hip mask HipM, shown in Figure 4.5. Two parameters were extracted from HipM to provide estimates of the dimensions of the participant. They consisted of the hip width HipW and hip depth HipD of the body in the seated position. These were determined by the major and minor axes of the ellipse with the same second-moments as HipM.

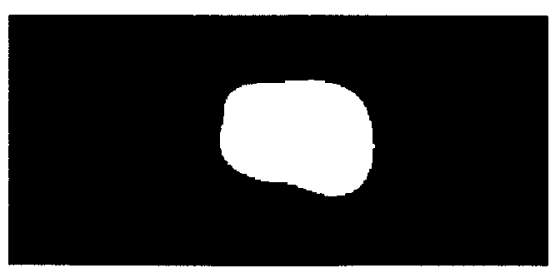

Figure 4.5: Binary hip mask HipM of a healthy participant. 


\subsubsection{Hand Region Detection}

\section{Sequential Frame Analysis}

Once the SiSt transfer began, the pressure exerted on the bed was expected to do two things: (1) decrease in the hip region as the body shifted the weight to its feet, and (2) increase in the hand region as they were used for support to lift from the bed.

The grayscale images of the transfer sequence were processed in consecutive pairs. The image in the current frame $I M G(f)$, as shown in Figure 4.6(a), was compared to image in the subsequent frame $\operatorname{IMG}(f+1)$, shown in Figure 4.6(b). All pixels found to decrease were placed in a new image $I M G_{d e c}(f)$, as shown in Figure 4.6(c), and those found to increase were placed in an additional image, $I M G_{\text {inc }}(f)$. Image $I M G_{\text {inc }}(f)$ is shown in Figure 4.6(d) after being amplified to improve visual recognition.

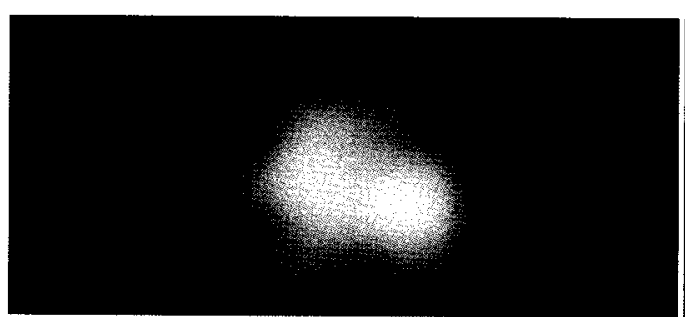

(a)

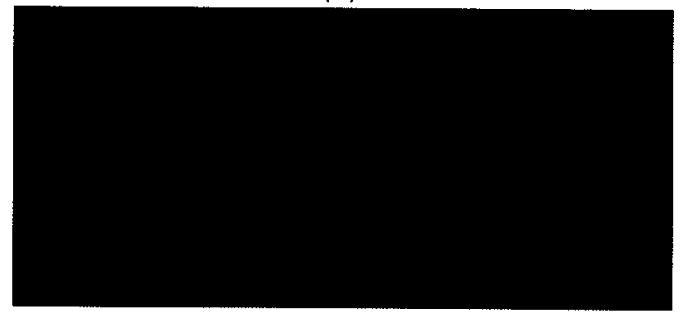

(c)

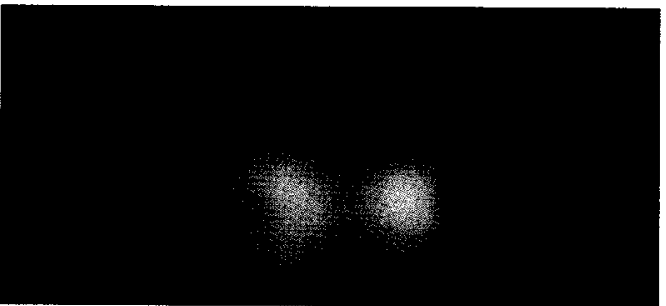

(b)

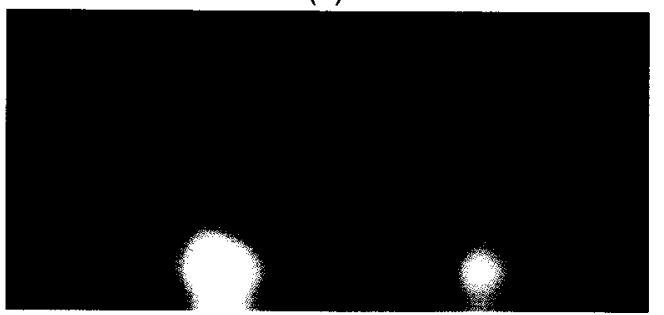

(d)

Figure 4.6: Sample of sequential frame analysis for a healthy participant (a) image in current frame $I M G(f)$, (b) image in subsequent frame $I M G(f+1)$, (c) image of decreasing pixels $I M G_{d e c}(f)$, and (d) amplified image of increasing pixels $I M G_{i n c}(f)$. 


\section{Region Definition}

The function of the hands during the SiSt transfer is to push down on the bed to gain momentum while maintaining balance in order to lift the rest of the body onto its feet. The image $I M G_{i n c}(f)$, which represented the increasing pressure placed on the bed during the transfer, was therefore expected to be composed mostly of pixels associated with the hand regions. Some pixels, however, were associated with noise caused by motion and therefore a denoising procedure was first investigated. Denoising is widely performed to remove noise artifacts from either a single signal [65], [66] or an image of interest [67], [68]. A study of the noise artifacts revealed an optimal denoising threshold of $T d_{o p t}=0.5$ and the pixels were subsequently converted to binary values. This resulted in a binary image $I M G_{R E G}(f)$ with all values above $T d_{o p t}$ set to one, while all values below were set to zero. At this point the image contained all detected regions, whether or not they were significant. Binary cluster detection was then performed to find the regions of interest $R E G(f, i)$ as shown in Figure 4.7. The CP location of each region $R E G_{C P x, y}(f, i)$ was extracted using its stored pixel locations and grayscale intensities, then saved as a potential hand to be considered in the verification step.

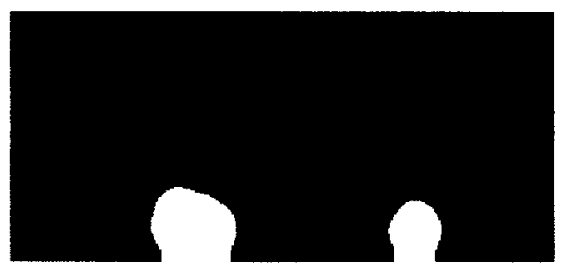

Figure 4.7: Binary image $I M G_{R E G}(f)$ containing regions $R E G(f, 1)$ and $R E G(f, 2)$. 


\section{Hand Region Verification}

While the $I M G_{R E G}(f)$ highlighted the increase in pressure from the hand regions, the forward motion of the SiSt also involved an increase in pressure from the thighs. In healthy transfers where the hands were used very lightly or not at all, this became even

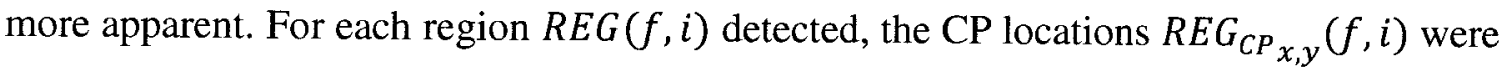
subsequently verified to determine their physical feasibility of belonging to the body's hands. Since hands and hips could not occupy the same space at the same time, boundaries were set according to the previously determined seated region parameters: center of pressure location $C P_{\text {sit }_{x, y}}$, hip width HipW, and hip depth, HipD. A $10 \%$ overlap in the $\mathrm{x}$-direction between the hands and hips was allowed to handle instances of the hands being temporarily placed directly under the hips at points throughout the transfer. To warrant further consideration, each $R E G_{C P, y}(f, i)$ needed to lie outside of the boundaries defined in the $\mathrm{x}$-direction by

and

$$
\begin{aligned}
& R E G_{C P_{x}}(f, i)>C P_{s i t_{x}}+[(1-O v l p) / 2] H i p W \\
& R E G_{C P_{x}}(f, i)<C P_{s i t_{x}}-[(1-O v l p) / 2] H i p W
\end{aligned}
$$

where $O v l p=10 \%$, and in the $\mathrm{y}$-direction by

$$
R E G_{C P}(f, i)>C P_{\text {sit }_{y}}+\operatorname{HipD} / 2
$$

where $H i p D / 2$ corresponded to the region of the thighs. Figure 4.8 depicts the seated region image $I M G_{\text {sit }}$ marked with its hip parameters and hand region boundaries. Figure 4.9 demonstrates the need for defining these boundaries, by displaying four sample $I M G_{R E G}(f)$ images with detected regions which fell outside of them and would otherwise 
have been considered hands. The HipM, seen as the top region in each image, has been added to the samples as a visual frame of reference.

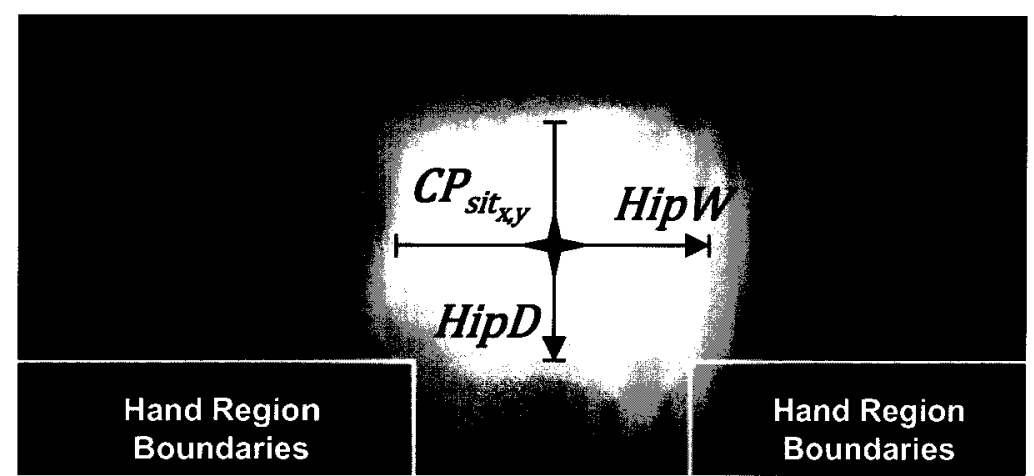

Figure 4.8: $I M G_{\text {sit }}$ with corresponding hip parameters and hand region boundaries.

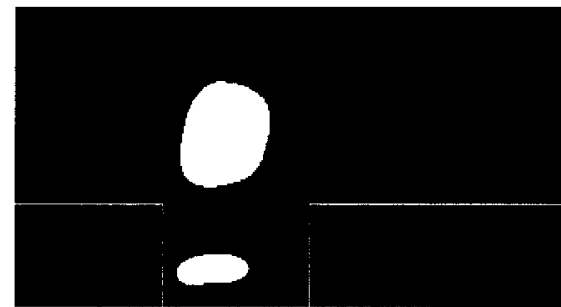

(a)

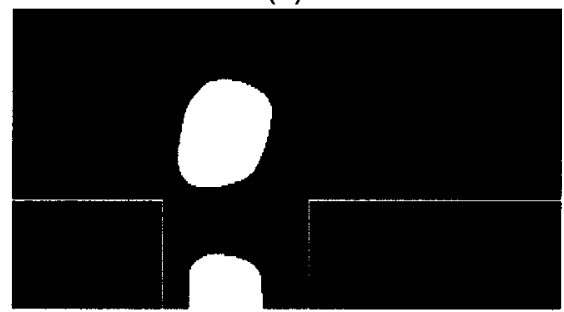

(c)

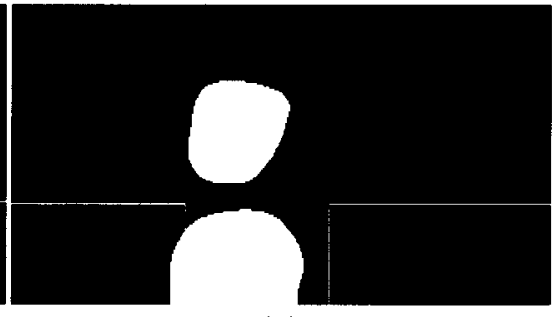

(b)

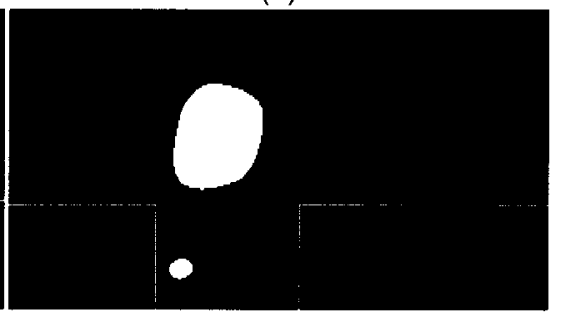

(d)

Figure 4.9: $I M G_{R E G}(f)$ displaying samples of regions outside of hand region boundaries.

\section{Hand Mask}

Each region $R E G(f, i)$ found adhering to the hand boundaries was kept as a cluster of ones in the binary image $I M G_{R E G}(f)$ and the rest were set to zeros. The $I M G_{R E G}(f)$ was then multiplied by its original $I M G_{i n c}(f)$ image pixels, reverting it back to grayscale. Contrary to the HipM which was set to provide binary rules of inclusion or exclusion of 
pixels into the detected region, the hand mask HandM was left in grayscale such that pixels which increased at a higher rate during the transfer had a higher weight than those that increased at a lower one. This was due to the faster transitions and wider range of motion of the hands throughout the SiSt sequence. The certainty of their pixel inclusion was lower in comparison to that of the hip region which was characterized by more constant frames at the start of the transfer segment. The multiplication was performed for all $F$ frames of the transfer segment, each incorporated into an average grayscale $H a n d M$ as shown in (4.4) and depicted in Figure 4.10.

$$
\operatorname{Hand} M=\frac{\sum_{f=1}^{F}\left(I M G_{i n c}(f) \times I M G_{R E G}(f)\right)}{F}
$$

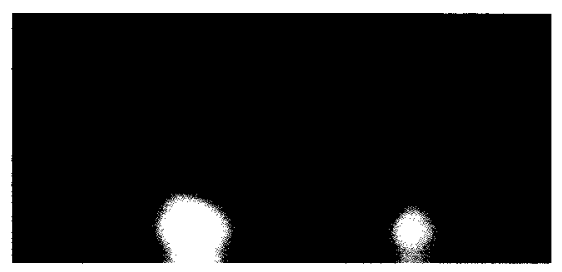

Figure 4.10: Grayscale hand mask $\operatorname{Hand} M$ of a healthy participant.

\subsection{Region of Interest Pressure Sequences and Feature Extraction}

\subsubsection{Regional Pressure Sequences}

With the hip and hand regions determined, sequences of regional pressure were extracted from the SiSt transfer. For each frame of the sequence, the HipM was multiplied by the $I M G(f)$ and summed to produce a hip pressure sequence as

$$
\operatorname{Hip} P(f)=\sum_{f=1}^{F} H i p M \times I M G(f) .
$$


The Hand $M$ was multiplied by each frame in the same manner. The pixels whose $\mathrm{x}$ coordinates, $\operatorname{Hand} M_{x}$, were located on the right side of the hip were assigned to the right hand and those on the left side of the hip to the left hand as follows:

$$
\operatorname{LHand} P(f)=\sum_{f=1}^{F} \operatorname{Hand} M \times I M G(f), \quad \operatorname{Hand}_{x}>C P_{\text {sit }_{x}}
$$

and

$$
R H a n d P(f)=\sum_{f=1}^{F} \operatorname{Hand} M \times I M G(f), \operatorname{Hand}_{x}<C P_{\text {sit }_{x}} .
$$

The regional pressure sequences $\operatorname{Hip} P(f), \operatorname{LHand} P(f)$, and $R \operatorname{Hand} P(f)$ were extracted from each transfer in the data set. Figure 4.11 displays the results of a transfer performed by a healthy adult, while Figure 4.12 displays that of a mobility-impaired participant.

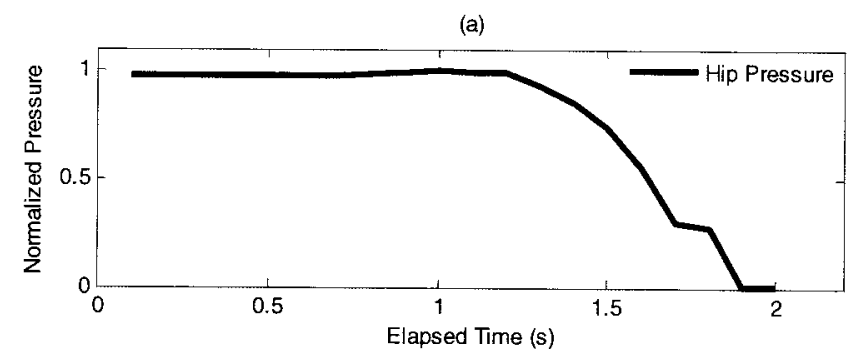

(b)

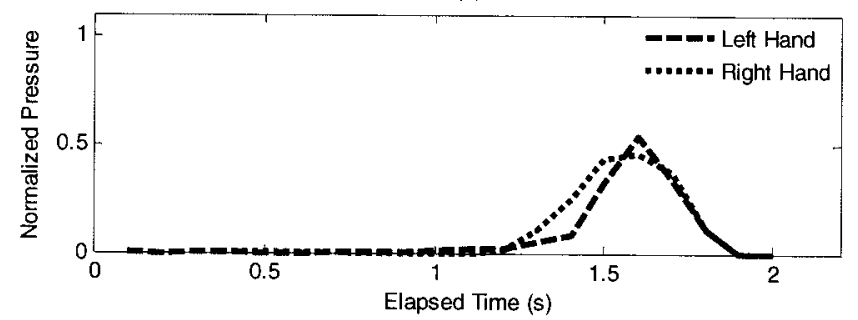

Figure 4.11: Healthy transfer pressure sequences from (a) hip region and (b) hand regions.

To enhance the visualization in the two figures, each hip sequence has been normalized by its maximum and the hand sequences have been normalized by the sum of their maxima. The regional pressure sequences allow the observation of the evolution of the pressure on the bed throughout the SiSt transfer. The hip sequence behaves as expected, 
with a slight increase as the body gains momentum and then a decrease over time as the body transitions to a stand. The hand regions of the healthy transfer remain fairly close to each other as seen in Figure 4.11. In the mobility-impaired transfer of Figure 4.12, the left hand immediately exerts pressure values near four times those of the right hand.
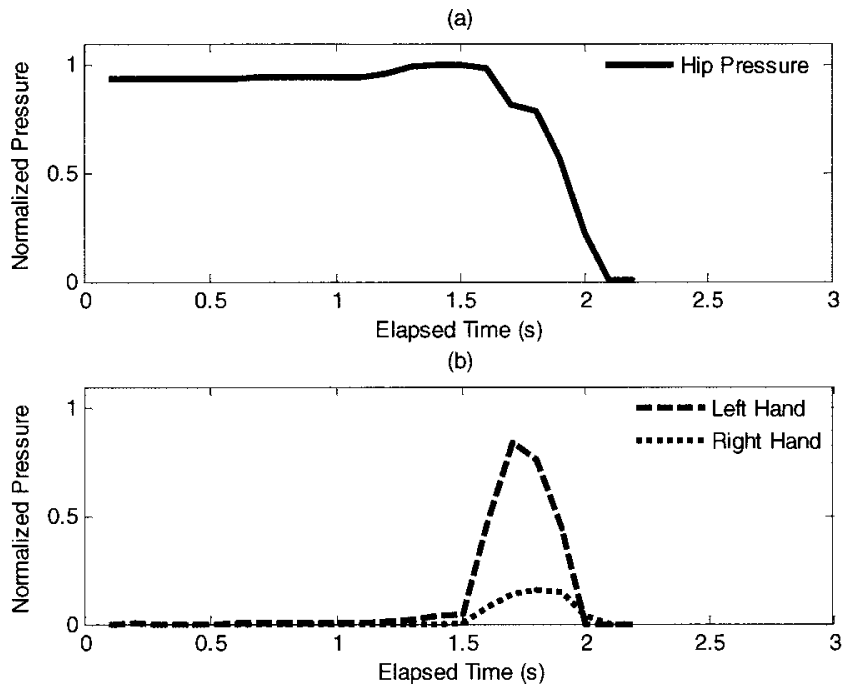

Figure 4.12: Mobility-impaired transfer pressure sequences from (a) hip region and (b) hand regions.

\subsubsection{Clinical Feature Definition}

In cooperation with the TAFETA clinical team, the following clinically relevant features were identified for the analysis of regions of interest during SiSt transfers:

1) Hand usage: whether left, right or both hands were used as aids to push off of the bed 2) Length of use: duration of time each hand was used to push off of the bed

3) Maximum pressure: maximum pressure placed on the bed by each hand, normalized by the occupant's weight

4) Average hand pressure difference: the difference between the pressure placed by the two hands, averaged over the SiSt sequence 
5) \% Hip decrease at maximum hand increase: percentage of decrease in the hip pressure at the point in which the hand pressure has reached a maximum

\subsubsection{Interactive Clinical Assessment Tool}

A prototype of a user-friendly interactive assessment tool was created for the clinical analysis and monitoring of SiSt transfers. As shown in Figure 4.13, it consisted of five modes of information retrieval: (1) data collection, (2) individual transfer analysis, (3) SiSt trends, (4) medical history and (5) mobility summary. The transfer analysis view contained the option of playing back the sequence of images as well as viewing the pressure sequences of the hips and hands. It also displayed the five clinical features extracted from the tested participants.

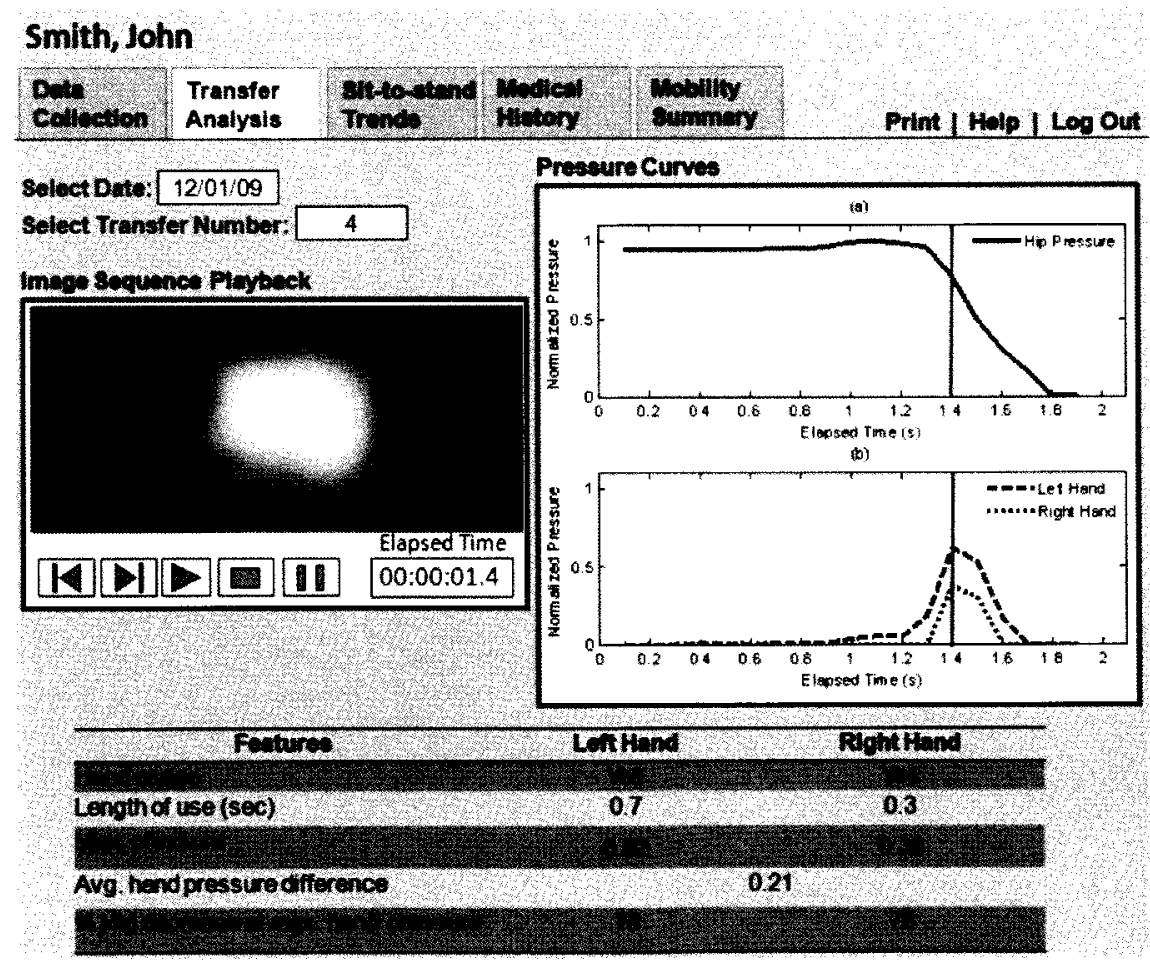

Figure 4.13: SiSt clinical assessment tool prototype: transfer analysis view containing image sequence playback, regional pressure sequences and extracted features. 
The SiSt trends view is displayed in Figure 4.14. It contained the same features extracted in the previous view, but with trend information displayed over time. The clinician could select whether they wanted to view the patient behaviour over the last day, week and/or month. They could then use this information to distinguish between normal and outlier behaviour, or observe the overall mobility trends of a patient over time. The month view in Figure 4.14 uses simulated data to demonstrate a trend in which the number of transfers and right hand usage remain fairly constant, but the left hand usage slowly decreases until it is no longer used to exit the bed. This could be due to muscle weakness or a loss of coordination, which if caught early could be treated and/or rehabilitated, decreasing the risk of falls leading to long-term impairment.

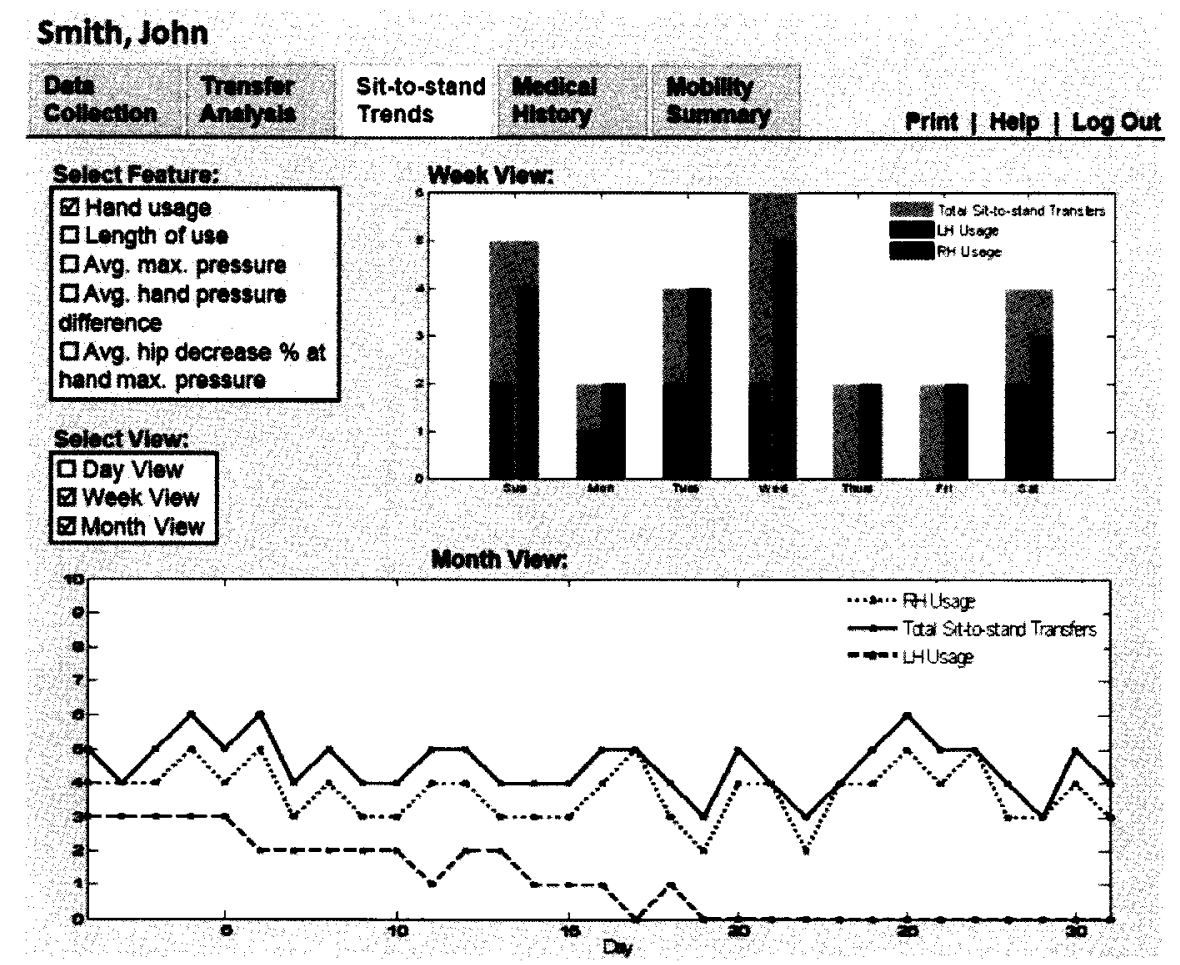

Figure 4.14: SiSt clinical assessment tool prototype: trends view containing the option of viewing the last day, week or month of extracted features over time. 


\subsubsection{Symmetry Measurement}

Following a hip-fracture or stroke, seniors are often faced with a significantly weaker side of the body than the other. Measurements of body symmetry have been made in recent studies using inertial sensors [69] and retro-reflective markers [70] worn on the body. The pressure sequences extracted from the hand regions can indicate instances of asymmetry in the body in an unobtrusive manner. An automated measurement of symmetry during the SiSt transfer was designed based on the evolution of the regional pressure sequences over time. The difference $\operatorname{diff} P(f)$ between the two hand pressure sequences was measured as

$$
\operatorname{diff} P(f)=R \operatorname{Hand} P(f)-L \operatorname{Hand} P(f) .
$$

From this difference sequence, the right and left hand dominant pressures RHdom and LHdom were measured as

and

$$
\begin{aligned}
& R H \text { dom }=\max (\operatorname{diff} P(f)) \\
& \text { LHdom }=\min (\operatorname{diff} P(f)) .
\end{aligned}
$$

The final symmetry decision was then based on the difference between the two dominant values being greater than a predefined threshold $d t h r$ as

$$
\begin{aligned}
& \text { RH asymmetry if }(\text { RHdom }- \text { LHdom })>d t h r \\
& \text { and } \quad \text { LH asymmetry if }(\text { RHdom }- \text { LHdom })<-d t h r .
\end{aligned}
$$

The threshold $d t h r=100$ was chosen based on the receiver operating characteristic (ROC) curve resulting from the tested data set. ROC curves are widely used in the 
selection of thresholds in intelligent systems [71]. Figure 4.15(a) displays the ROC curve of True Positive versus False Positive Rates of symmetry classification. Figure 4.15(b) displays the distance between $[0,1]$ and each point on the ROC curve. The minimum distance between these points, considered the optimal operating point in classification of the tested data set, is highlighted by the red arrows in the two figures.

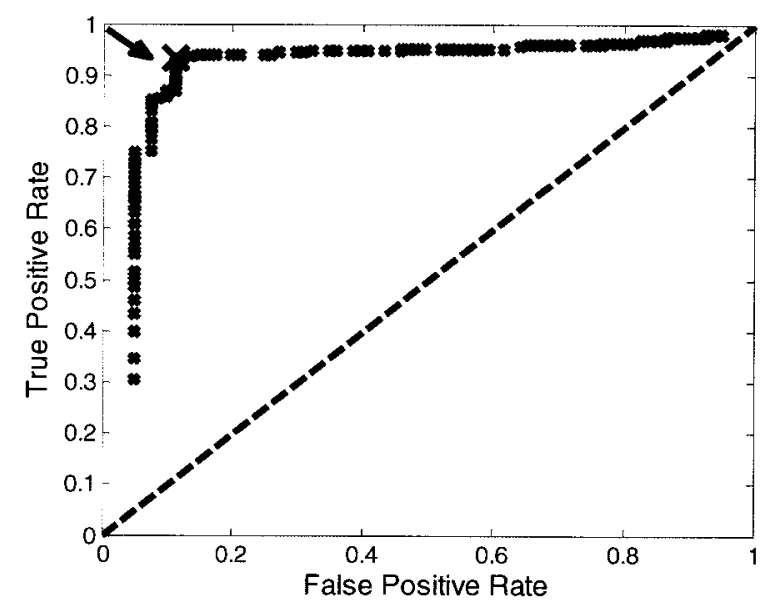

(a)

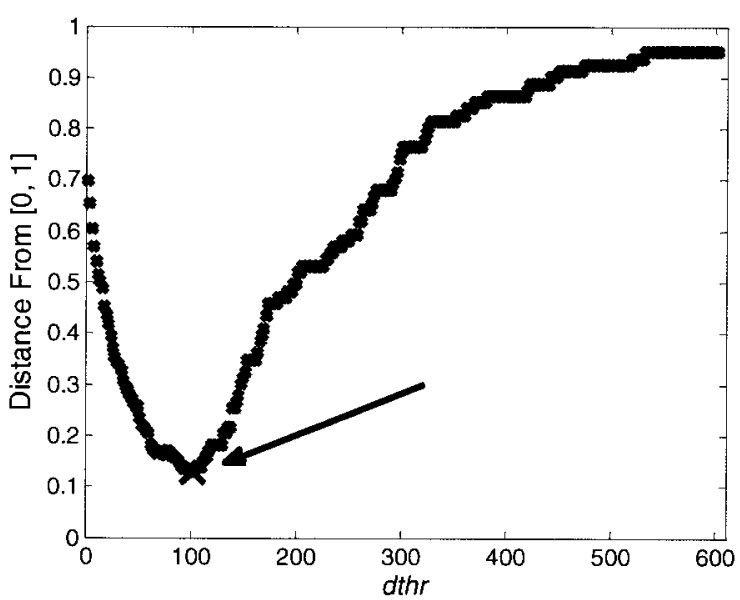

(b)

Figure 4.15: Selection of symmetry threshold by considering (a) the ROC curve and (b) the distance between $[0,1]$ and the ROC curve.

\subsection{Results and Verification}

\subsubsection{Abnormal Transfer Visibility}

As introduced in Chapter 3, the SiSt transfers were assessed by the TAFETA clinical team by reviewing the recorded videos frame by frame. This allowed them to observe characteristics that would not have otherwise been visible through in-vivo analysis alone. All transfers were characterized and documented into a database by their relevant characteristics, allowing for system verification. Further to this, the regional pressure sequences extracted from the automated algorithm presented in this chapter provided a 
more detailed view of characteristics which were initially undetected in the video analysis but subsequently confirmed by a secondary review by the clinical team. In this section, two such characteristics presented. Both were considered by the clinical team to be out of the ordinary and worthy of further attention: (1) single-hand use and (2) bouncing.

\section{Single-Hand Use}

In more extreme cases of discomfort or paralysis in the body, a single side of the body alone may be functional during the SiSt transfer. An example of this is shown in Figure 4.16. This sequence was recorded from a post-stroke participant who had lost the use of the right side of their body. While this would perhaps be obvious visually, a gradual decrease in the strength of one side could be unobtrusively perceived by a system of ongoing monitoring over time.
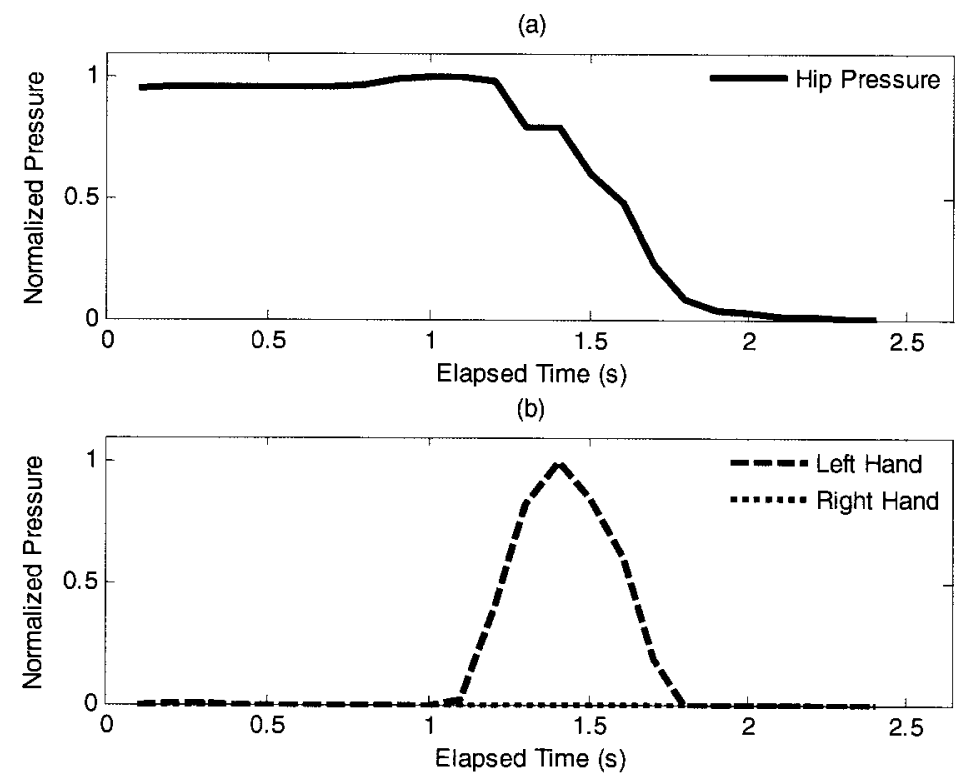

Figure 4.16: Single-handed transfer pressure sequences from (a) hip region and (b) hand regions. 


\section{Bouncing}

A common indication of loss of strength and coordination is the occurrence of bouncing or unsuccessful attempts during the SiSt transfer. These occur when a person initiates the SiSt with the intention of standing, but due to insufficient strength or a loss of balance they are forced to sit back down. A sample from a post-stroke participant is shown in Figure 4.17. The characteristic can be seen in the hip sequence of Figure 4.17(a) as a set of peaks and valleys before the final lift of the body off of the bed. The characteristic is even more pronounced in the left hand sequence of Figure 4.17(b), where the pressure begins to increase but falls back to zero in several consecutive failed transfer attempts.

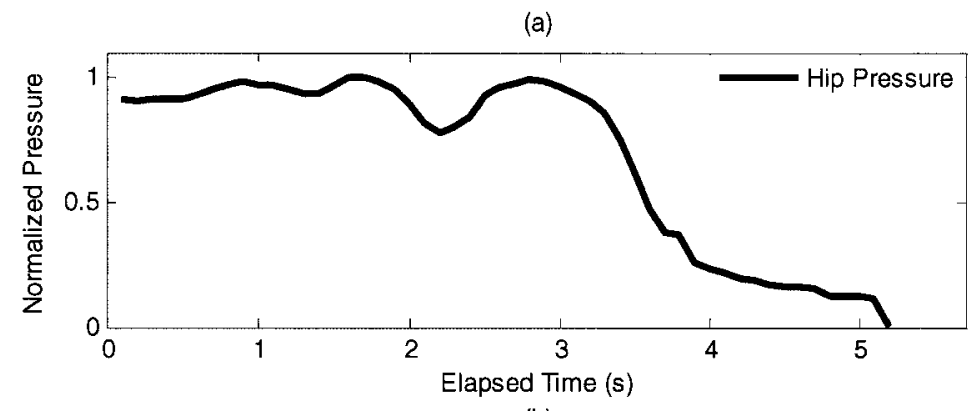

(b)

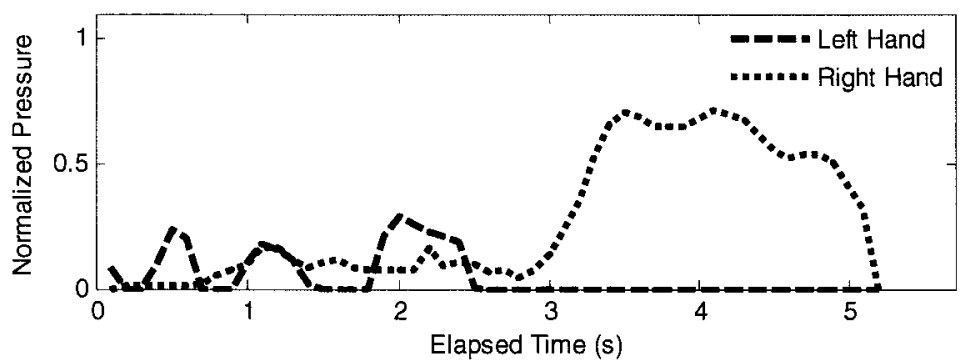

Figure 4.17: Bouncing transfer pressure sequences from (a) hip region and (b) hand regions.

\subsubsection{Symmetry Classification}

The symmetry estimation algorithm results were verified by the clinical team's SiSt transfer database, as shown in Table 4.1. The 'Video Output' refers to the video 
verification performed by the clinical team, and the 'Algorithm Output' refers to those of the symmetry algorithm presented in Section 4.4.4. The results show that based on 287 tested transfers, the algorithm was capable of automatically classifying symmetry with an overall accuracy of $93.0 \%$. The sensitivity, defined by the correct classification of symmetrical transfers, resulted in $93.0 \%$ and the specificity, defined by the correct classification of asymmetrical transfers, was of $93.5 \%$. The overall accuracy, defined by the percentage of correctly classified transfers overall, was found to be $93.0 \%$

TABLE 4.1: RESULTS OF SYMMETRY CLASSIFICATION DURING SIST TRANSFERS

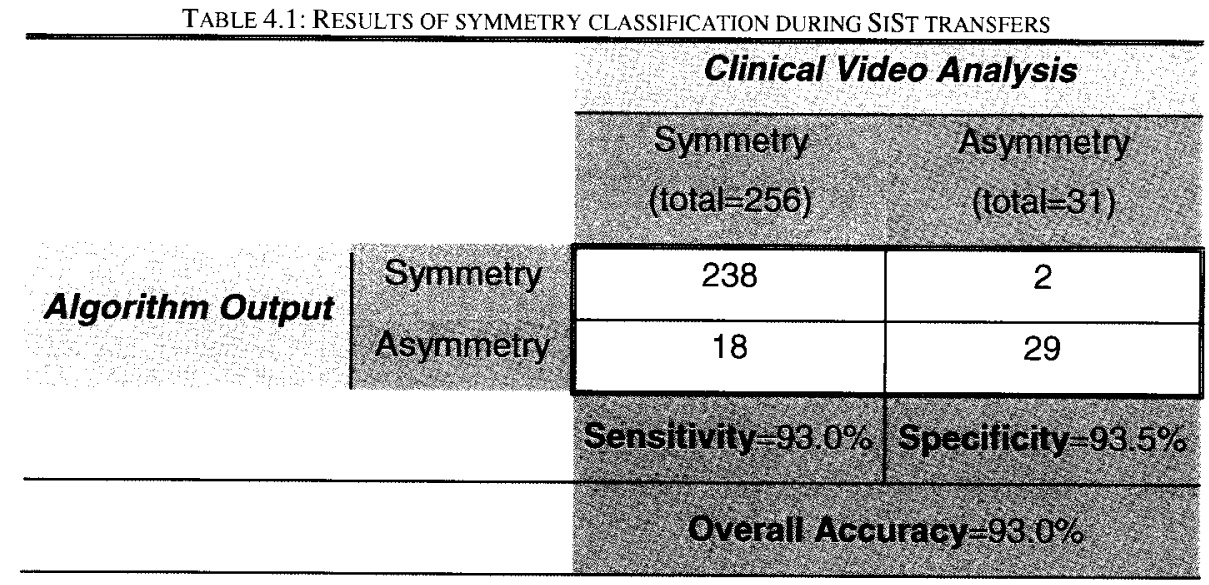

\subsection{Discussion}

This chapter has presented the successful extraction of features which in clinical practice are presently observed visually and are subject to a high degree of subjectivity. A current limitation of the symmetry classification stems from occasional confusion in the detection of the regions of interest. When a hand was placed either too close or on top of the thigh rather than beside it, the hand was occasionally confused for part of the hip region during parts of the SiSt transfer. Also, while the symmetry measurements provided a successful classification method based on healthy participants, it is expected that there may be additional challenges introduced by the behaviour of patients with more 
pronounced mobility impairments. Sliding in a horizontal direction during the SiSt or using body parts other than the hands to push off of the bed have not yet been considered and should be addressed in related future work. Additional data collection would help verify whether the current results are representative of the entire population. Finally, it is worth noting that the accuracy of the symmetry classification was based on a comparison to the clinical video verification. It is expected, however, that the sensors will produce a more precise measurement of symmetry than the visual analysis that is currently the gold standard in clinical practice.

\subsection{Conclusions}

In this chapter, an algorithm has been designed to automatically detect the regions of interest and extract features which characterize SiSt transfers based on sequences of pressure images. The algorithm utilized the differences between consecutive images to construct ROI masks. The resulting regional pressure sequences allowed the observation of the SiSt motion over time and the extraction of features of interest as well as the automated measurement of symmetry. Data collected from both healthy and mobilityimpaired participants were analyzed, with several cases of abnormal transfers found and subsequently verified by the TAFETA clinical team. It is expected that as part of an ongoing monitoring system, the clinical features extracted by the ROI-based algorithm would allow the automated detection of changes in mobility over time. 


\section{Chapter 5:}

\section{Sagittal Center of Pressure Trajectory Analysis from Bed Pressure Sequences}

\subsection{Introduction}

This chapter provides an algorithm to analyze the trajectory of the center of pressure (CP) during SiSt transfers for the purpose of detecting impaired mobility. It begins by introducing the properties of the SiSt CP in Section 5.2. In Section 5.3, samples recorded from both healthy and mobility-impaired transfers during clinical trials are characterized and the features of interest are defined. An algorithm for the automated detection of mobility-impaired transfers based on the trajectory of the sagittal CP is then presented. Section 5.4 reports the results of the features extracted along with their relative significance and the automated transfer classification. A discussion follows in Section 5.5 and the chapter concludes in Section 5.6. Figure 5.1 depicts the focus of this chapter within the monitoring system design. 


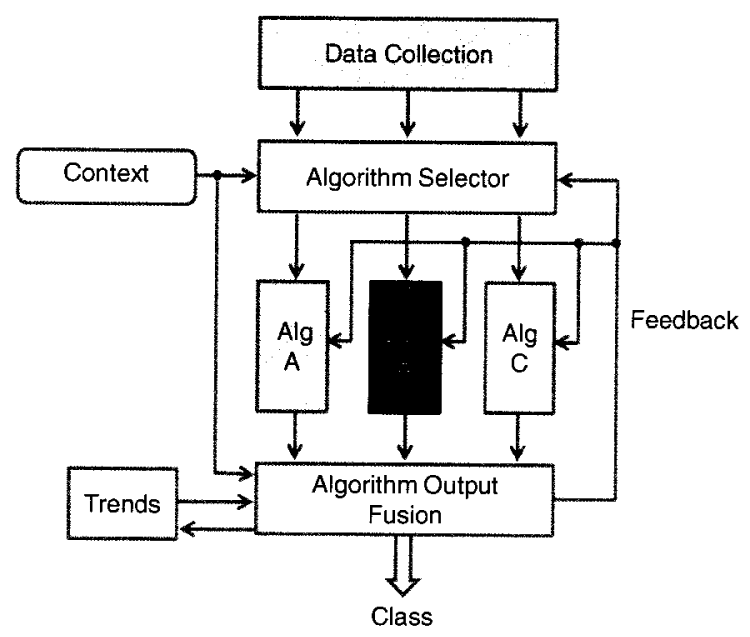

Figure 5.1: Monitoring system feature extraction algorithm B.

\subsection{Properties of the Center of Pressure Trajectory During Sit-to-Stand}

For the current bed-exit analysis, the coordinates of the $\mathrm{CP}$ are defined as $C P_{x}$ and $C P_{y}$ along two perpendicular planes. The first is a coronal plane which divides the body into anterior and posterior parts and the second is a sagittal plane which divides the body into left and right halves. Figure 5.2 displays four photos and corresponding pressure images during a healthy subject's bed-exit. An increase over time in the $C P_{y}(n)$ signifies a shift of pressure towards the anterior edge of the bed, whereas a decrease signifies a shift back to its posterior edge. In the image of Figure 5.2(a), the initial seated position is seen in the form of a white oval representing the bulk of the hip pressure on the bed and two faded regions resulting from the pressure of the thighs. In Figure 5.2(b) a visible shift in pressure is seen towards the anterior of the bed as the subject leans forward. Figure 5.2(c) displays most of the pressure very close to the anterior edge of the bed as the subject is in a near seat-departure position. In Figure 5.2(d) the subject has reached a standing position, all pressure is lifted off the mats and no pressure regions are visible. Contrary to 
the ROI algorithm in Chapter 4, the behaviour of the hands is not specifically considered in the CP trajectory analysis. This allows feature extraction from those who either do not use their hands during bed-exit or for those who place them on their thighs. It also aligns with the goal of designing a monitoring system which is flexible and adaptable to an occupant's needs and situational context.

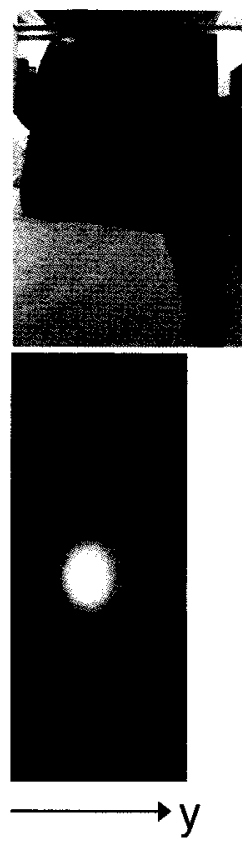

(a)
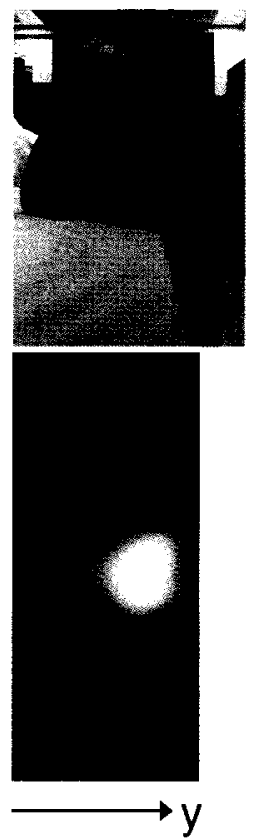

(b)
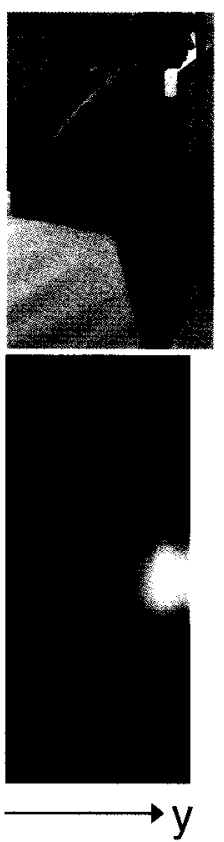

(c)

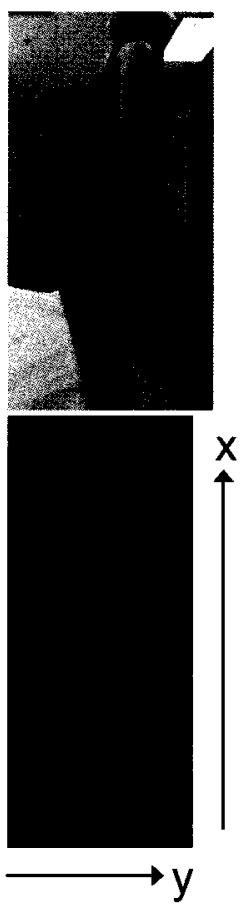

(d)

Figure 5.2: SiSt transfer sequence with corresponding pressure images.

\subsection{Center of Pressure Sequences and Feature Extraction}

For each transfer, the sensor outputs at time sample $n$ were extracted from the pressure mats and assigned to a spatial matrix using the mapping files provided by Tactex Controls Inc. Preliminary denoising was performed and the CP was determined based on 
the sensor outputs $s_{k}(n)$ and their originating locations $\left(x_{k}, y_{k}\right)$. The two coordinates $C P_{x}(n)$ and $C P_{y}(n)$ of the center of pressure trajectory at time sample $n$ were measured as

$$
\begin{aligned}
& C P_{x}(n)=\frac{\sum_{k=1}^{S} s_{k} x_{k}}{\sum_{k=1}^{S} s_{k}} \\
& C P_{y}(n)=\frac{\sum_{k=1}^{S} s_{k} y_{k}}{\sum_{k=1}^{S} s_{k}}
\end{aligned}
$$

Following are some of the characteristics observed in the $C P_{y}(n)$ trajectories during the bed-exits from both healthy and mobility-impaired groups.

\subsubsection{Sagittal Trajectory Sequences}

Samples of the $C P_{y}(n)$ trajectories for two healthy participants are displayed in Figure 5.3. As expected, the sequences increase over time as the bodies lean forward to perform the SiSt transfer off of the bed. In Figure 5.3(a), the participant starts at $35 \mathrm{~cm}$ from the posterior edge of the bed and reaches the point of seat-departure after a forward displacement of $5 \mathrm{~cm}$ after an elapsed time of $1.2 \mathrm{~s}$. The participant in Figure 5.3(b) has approximately the same amount of displacement but completes it in a shorter time of approximately $0.6 \mathrm{~s}$.

Figure 5.4 depicts the $C P_{y}(n)$ trajectories for two mobility-impaired participants. These sequences began with an increase over time but, due to a lack of strength or coordination, deviated from a linear path before reaching seat-departure. The sample in Figure 5.4(a) shows a clear sagittal rocking motion between 2.8 and 3.5 seconds. In Figure 5.4(b), a first attempt at standing consisted of a forward motion but ended with a 
failure at $1.4 \mathrm{~s}$. A second attempt is seen at $2.2 \mathrm{~s}$, which led to a successful departure off of the bed.

(a)

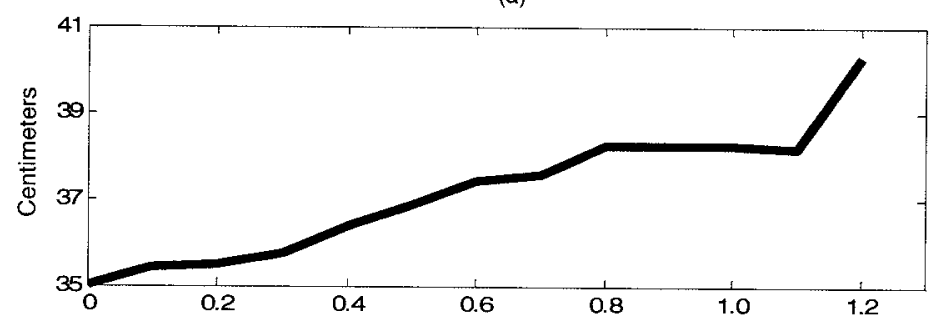

(b)

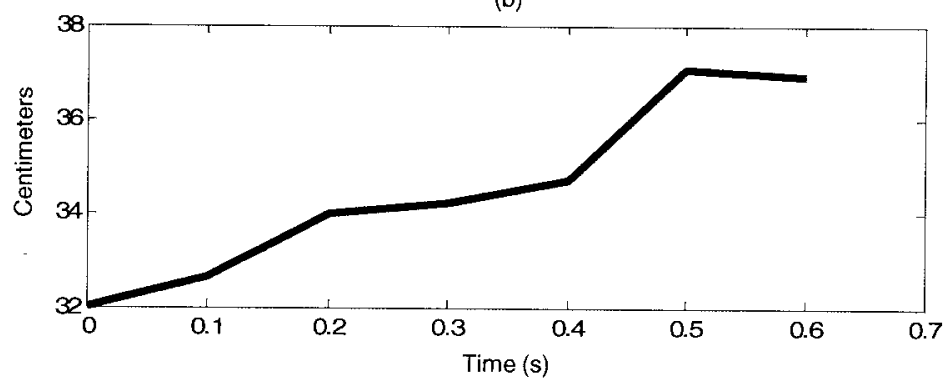

Figure 5.3: Measured $C P_{y}(n)$ trajectories during the SiSt of two healthy participants.

(a)
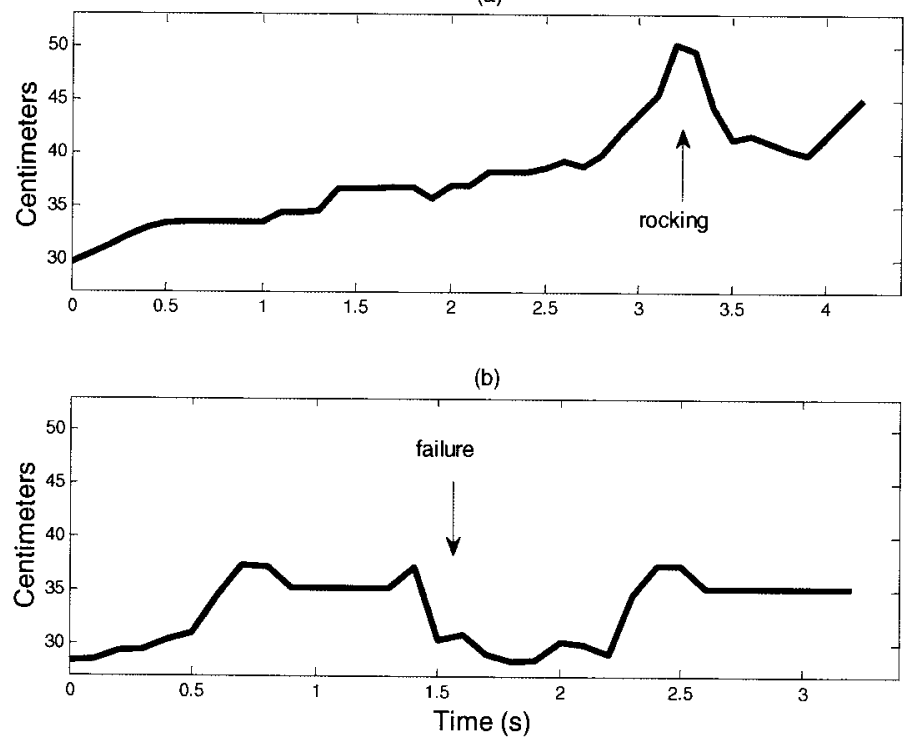

Figure 5.4: Measured $C P_{y}(n)$ trajectory for mobility-impaired participants, exhibiting characteristics of (a) rocking by a participant post-hip-fracture and (b) SiSt failure by a participant post-stroke. 


\subsubsection{Clinical Feature Definition}

In conjunction with the TAFETA clinical team, the following clinically relevant features were identified for the analysis of the CP trajectories during SiSt transfers:

1) Sagittal deviation: measure of the CP deviation in the sagittal (y-axis) direction from the start of the SiSt transfer to the point of seat-departure

2) Seat-departure duration: time from the start of the SiSt transfer to the full departure of the body from the bed

3) Initial position: location of the $\mathrm{CP}$ in relation to the anterior edge of the bed at the start of the SiSt transfer

4) Forward sagittal displacement: measure of the sagittal displacement of the body from the initial position to the anterior edge of the bed before seat-departure

5) Coronal deviation: measure of the $\mathrm{CP}$ deviation in the coronal ( $\mathrm{x}$-axis) direction from the start of the SiSt transfer to the point of seat-departure

\subsubsection{Sagittal Deviation Measurement}

The sagittal deviation measurement begins with the assumption that an ideal $C P_{y}(n)$ trajectory exhibits a linear behaviour, shown in Figure 5.5(a), as the healthy body moves forward with no hesitation directly off of the bed. A mobility-impaired body, depicted in Figure 5.5(b), may then be characterized by the measurement of deviation of the sequence from that of a straight line.

The system architecture describing the algorithm for the classification of impaired transfers is depicted in Figure 5.6. The objective of the classification algorithm was to measure deviations in motion from the linear trend of the ideal SiSt. The first step was to 


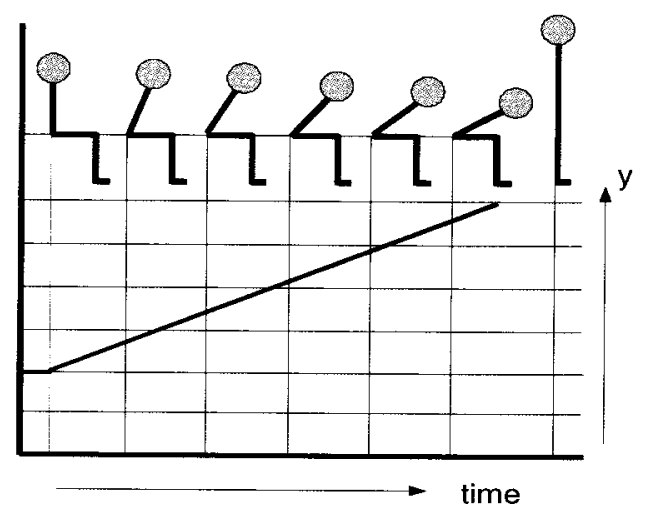

(a)

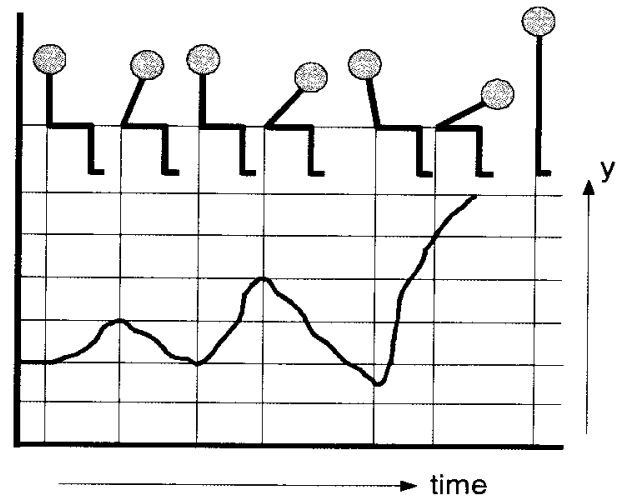

(b)

Figure 5.5: Simulated $C P_{y}(n)$ trajectory for SiSt transfers of (a) healthy and (b) mobilityimpaired participants.

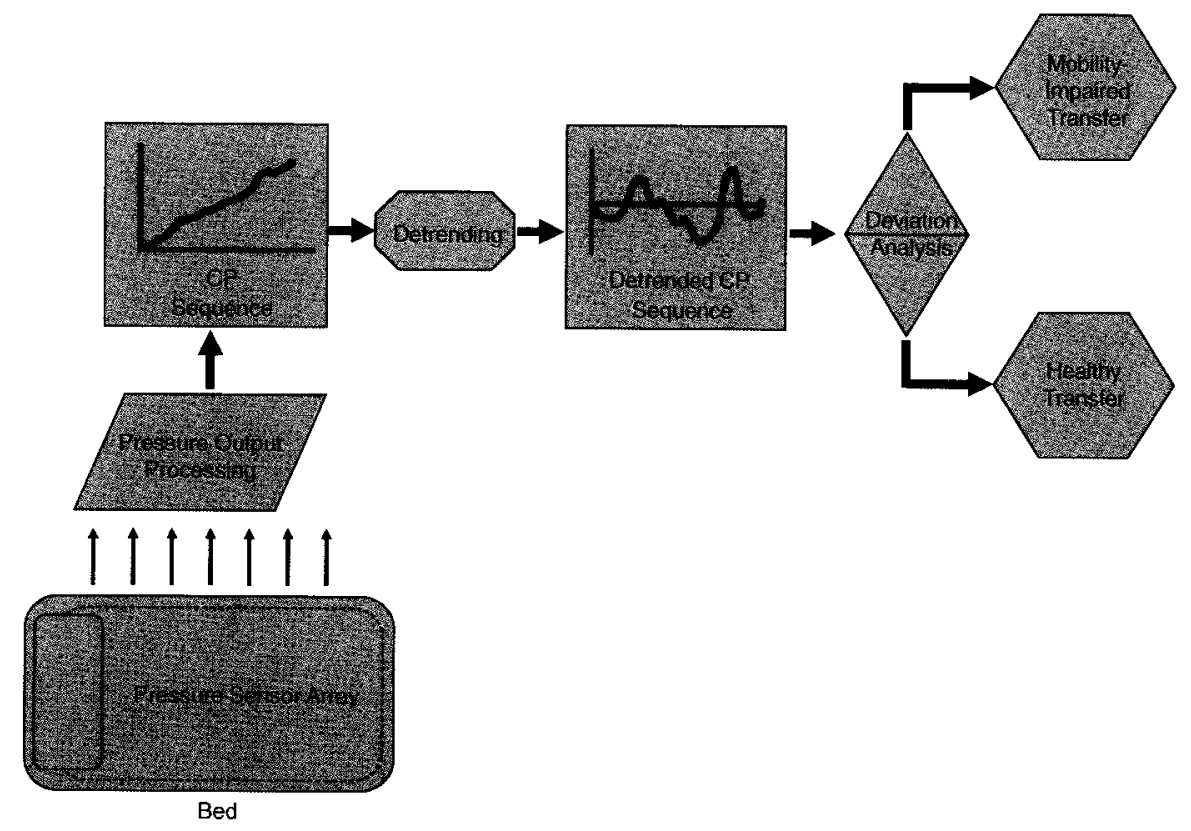

Figure 5.6: System architecture of mobility-impaired SiSt transfer classification from bed pressure sequences.

detrend the $C P_{y}(n)$ trajectory. Detrending is largely performed to remove the slow varying trend in a signal in order to observe its faster varying characteristics [72], [73]. 
This was accomplished by approximating each center of pressure trajectory $C P_{y}(n)$ to a linear function as

$$
f_{\text {lin }}(V, n)=v_{0}+v_{1} n
$$

where $v_{0}$ and $v_{1}$ are the coefficients of the linear approximation. An error function was defined as

$$
\mathrm{e}(n)=C P_{y}(n)-f_{\text {lin }}(V, n)
$$

and the optimal coefficients $V_{o p t}=\left[v_{0_{o p t}}, v_{1_{o p t}}\right]$ were determined such that $\mathrm{e}(n)$ was minimized in the least squares sense as

$$
\mathrm{e}_{\min }(n)=\operatorname{argmin}_{V_{o p t}} \sum_{n=1}^{N}\left[C P_{y}(n)-f_{\text {lin }}(\mathrm{V}, n)\right]^{2} .
$$

The approximated function with optimal coefficients was then subtracted from $C P_{y}(n)$, to leave the detrended sequence

$$
C P_{y_{d t r}}(n)=C P_{y}(n)-f_{\text {lin }}\left(V_{o p t}, n\right)
$$

The standard deviation of the detrended $C P_{y_{d t r}}(n)$ sequence was used as the measure of sagittal mobility within the transfer. For each transfer, the deviation measure became

$$
\sigma_{y_{d t r}}=\sqrt{\frac{1}{N-1} \sum_{n=1}^{N p}\left(C P_{y_{d t r}}(n)-\overline{C P_{y_{d t r}}}\right)^{2}}
$$

where $\overline{C P_{y_{d t r}}}$ is the mean of the detrended sequence with length $N$. Figure 5.7 and Figure 5.8 display samples of the $C P_{y}(n)$ sequence and of the detrended sequence $C P_{y_{d t r}}(n)$ for one healthy transfer and one mobility-impaired transfer respectively. 

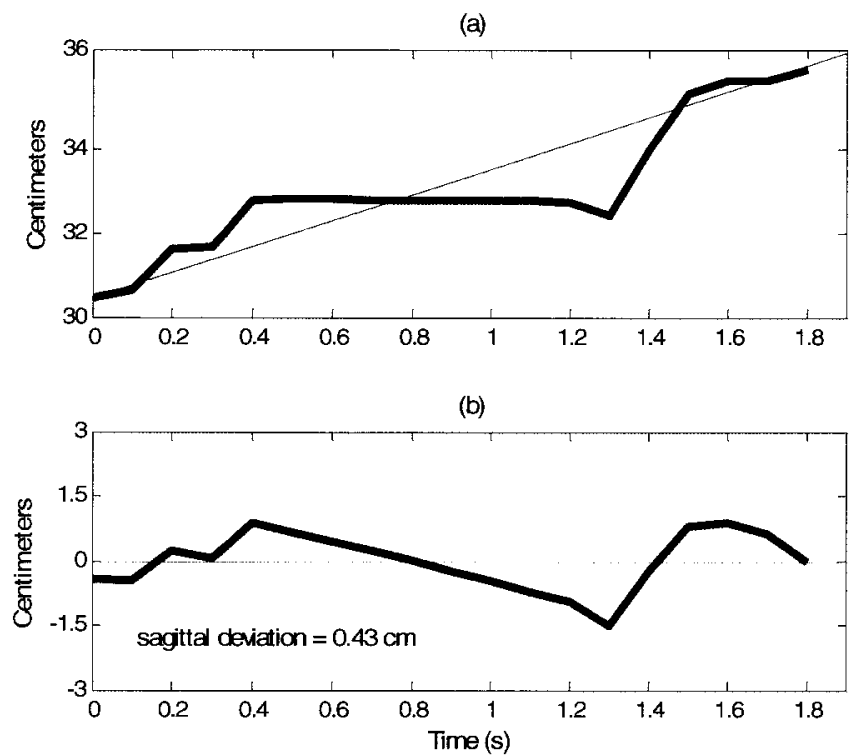

Figure 5.7: Measured sequences for a healthy participant (a) $C P_{y}(n)$ before detrending and (b) $C \boldsymbol{P}_{\boldsymbol{y}_{d t r}}(\boldsymbol{n})$ after detrending.
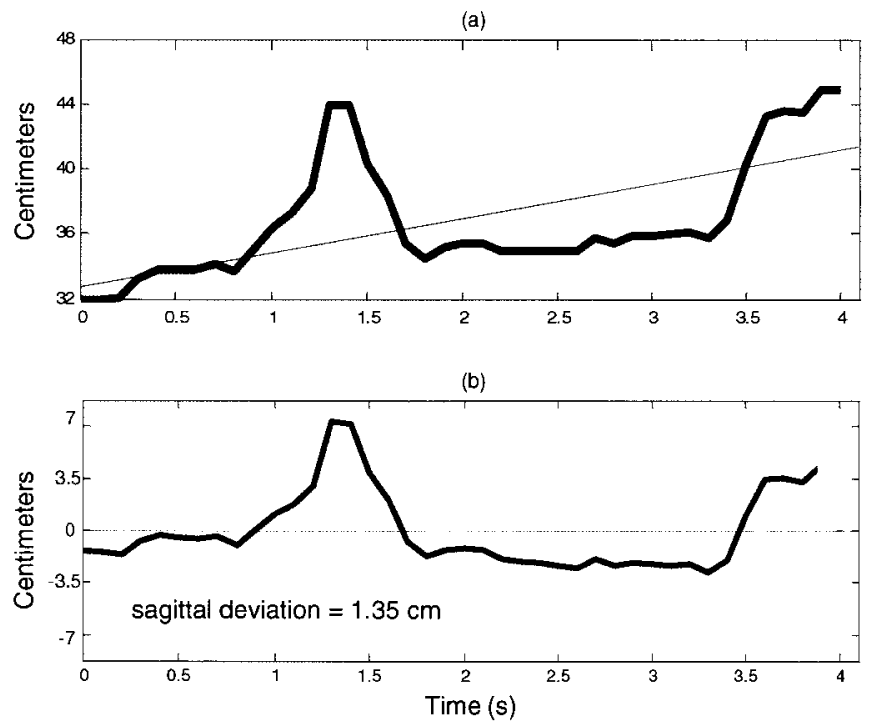

Figure 5.8: Measured sequences for a post-hip-fracture participant (a) $C P_{y}(n)$ before detrending and (b) $C P_{y_{d t r}}(n)$ after detrending.

The measures of all transfers were then compared to a threshold, vthr, to categorize them as healthy or mobility-impaired. As shown in Figure 5.9, the threshold $v t h r=0.39$ 
was chosen based on the receiver operating characteristic (ROC) curve resulting from the tested data set. Figure 5.9(a) displays the ROC curve of the True Positive versus False Positive Rates of sagittal deviation classification. Figure 5.9(b) displays the distance between $[0,1]$ and each point on the ROC curve. The minimum distance between these points, considered the optimal operating point in classification, is highlighted by the red arrows in the two figures.

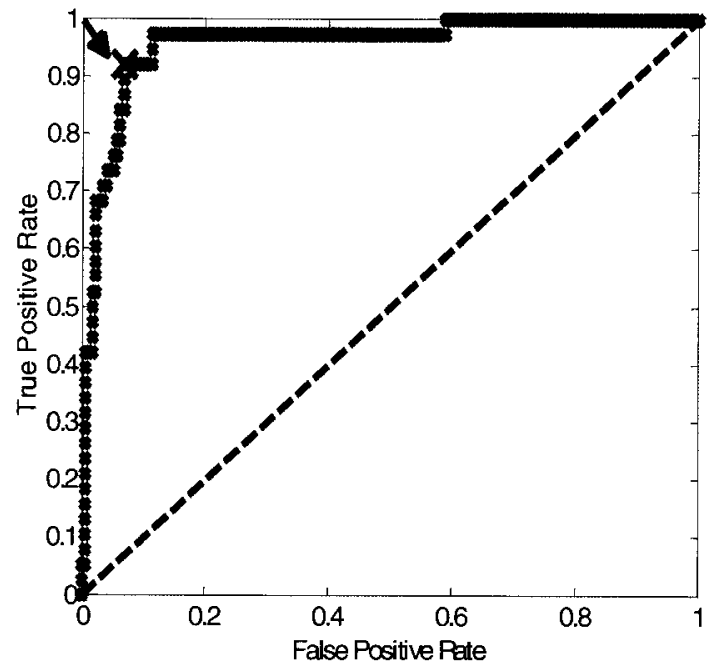

(a)

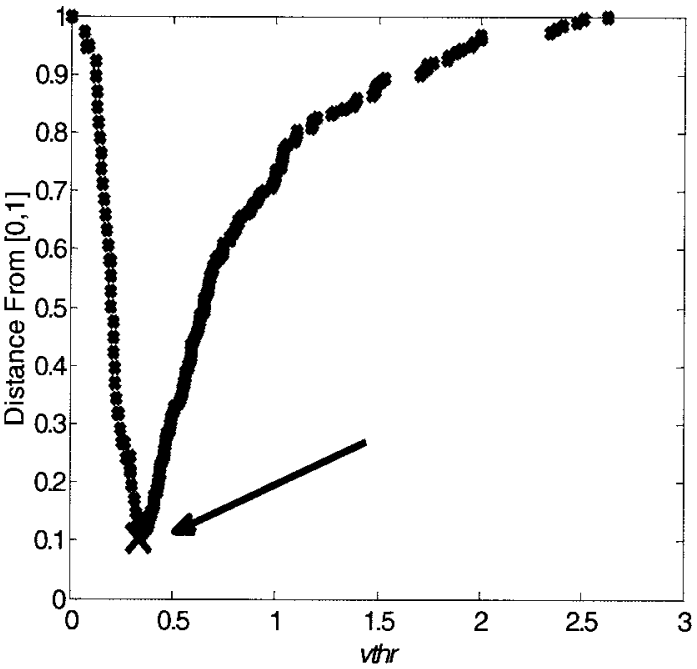

(b)

Figure 5.9. Selection of sagittal deviation threshold $v t h r$ by considering (a) the ROC curve and (b) the distance between $[0,1]$ and the ROC curve.

\subsection{Results and Verification}

\subsubsection{Extracted Clinical Features}

The clinical features which were defined in Section 5.3.2 were successfully extracted from the CP data and are reported in Table 5.1. The feature label can be found in the first column followed by the mean and standard deviations of the healthy and mobilityimpaired groups in the second and third columns. The final column contains the p-value 
resulting from a Kruskal-Wallis analysis of variance (ANOVA) test, under the assumption that the groups have the same continuous distribution and that the observations are mutually independent [74]. The difference between the tested groups is considered to be significant if the p-value is less than 0.05 .

TABLE 5.1: RESUlTS OF SAGITTAL CP FEATURES EXTRACTED FROM SIST TRANSFERS

\begin{tabular}{cccc}
\hline Feature & Healthy & Mobility- Impaired & p-value \\
\hline $\begin{array}{c}\text { Sagittal Deviation (cm) } \\
\sigma_{y_{d t r}}\end{array}$ & $0.48 \pm 0.23$ & $1.56 \pm 0.51$ & $<0.001$ \\
$N$ & $2.62 \pm 0.97$ & $4.72 \pm 1.38$ & $<0.001$ \\
$\begin{array}{c}\text { Seat-Departure Duration (s) } \\
N\end{array}$ & $33.79 \pm 2.38$ & $27.11 \pm 3.68$ & $<0.001$ \\
$\quad \begin{array}{c}C P_{y}(1) \\
\text { Initial Position (cm) }\end{array}$ & $4.58 \pm 3.61$ & $12.08 \pm 3.93$ & $<0.001$ \\
\hline $\begin{array}{c}\text { Forward Sagittal Displacement (cm) } \\
\left\{C P_{y}(n)\right]_{\text {max }}-C P_{y}(1)\end{array}$ & $1.85 \pm 1.21$ & $3.99 \pm 1.64$ & $<0.001$ \\
\hline $\begin{array}{c}\text { Coronal Deviation (cm) } \\
\sigma_{x_{d t r}}\end{array}$ & & & \\
\hline
\end{tabular}

These results were also found to be relevant in recently published clinical studies using less unobtrusive methods of data collection. The Duration of the SiSt transfer has been widely identified as a significant factor in transfer evaluation [75]. Using reflective markers and ground force plates, Mathiyakom et al. [41] found that making changes to the Initial Position of sitting participants altered the mechanical demands and the lower body kinetics of the SiSt transfer. They also found that reducing Forward Sagittal Displacement influenced the momentum requirements and resulted in significantly smaller horizontal velocities at seat-departure. The last feature reported in Table 5.1, Coronal Deviation, provides the same information as the Sagittal Deviation, but along the 
coronal plane. It is derived by the same procedure of linear approximation and detrending but focuses on the $C P_{x}(n)$ sequence instead of $C P_{y}(n)$. A high standard deviation can be due to an imbalance between the left and right side of the body, for instance due to pain or numbness, as well as to pressure placed on the bed by the arms to maintain mobility during the transfer. The mobility literature refers to a similar measure termed lateral or medio-lateral sway but typically it refers to the phase beginning after seat-departure and is generally measured by force plates under the feet [42],[45]. The reported quantities produced similar results with respect to the existence of significant differences between healthy and mobility-impaired groups. While the feature results in this chapter are promising, further tests with new samples are needed to help elucidate whether they can be interpolated to the entire population.

\subsubsection{Relative Significance of Extracted Clinical Features}

Binary logistic regression can be used to model a system in which a dependent variable has only two possible outcomes [76]. With the outcomes defined as healthy or mobilityimpaired, a regression was run using the five features extracted in this chapter as independent variables. A forward stepwise method was chosen which constructs the model by analyzing each variable individually for its contribution and adding in only those deemed statistically significant to the outcome. The regression identified the $\sigma_{y_{d t r}}$ feature as the most significant and concluded in one step. The first column of Table 5.2 contains the feature under consideration and the second contains the resulting score statistic assigned to each feature relative to its importance. The final column contains the 
relative significance of each when included in the model, typically considered statistically significant if less than or equal to 0.05 .

TABLE 5.2: RESULTS OF STEPWISE BINARY LOGISTIC REGRESSION FOR CLINICAL FEATURES

\begin{tabular}{|c|c|c|}
\hline Feature & Score & Significance \\
\hline $\begin{array}{l}\text { Sagittal Deviation (cm) } \\
\qquad \sigma_{y_{d t r}}\end{array}$ & 83.43 & $<0.001$ \\
\hline $\begin{array}{l}\text { Initial Position }(\mathbf{c m}) \\
\qquad C P_{y}(1)\end{array}$ & 3.56 & 0.06 \\
\hline $\begin{array}{c}\text { Seat-Departure Duration (s) } \\
\qquad N\end{array}$ & 2.85 & 0.09 \\
\hline $\begin{array}{l}\text { Coronal Deviation (cm) } \\
\qquad \sigma_{x d t r}\end{array}$ & 1.68 & 0.20 \\
\hline $\begin{array}{l}\text { Forward Sagittal Displacement (cm) } \\
\qquad\left[C P_{y}(n)\right]_{\max }-C P_{y}(1)\end{array}$ & 0.60 & 0.44 \\
\hline
\end{tabular}

These results highlight several points of interest. The first is that the Sagittal Deviation feature remains the most significant in identifying the mobility-impaired transfers, producing a significance value of less than 0.001 . The second is that the results obtained by the methods designed in this thesis agree with those found in previous related studies. The Initial Position feature was found to be very near to statistical significance, similar to the results found in [41]. The Seat-Departure Duration feature was also found to be close, as found in the results of [40] and [16]. Both of these features ought to be considered in future work on SiSt transfer analysis. Finally, it is shown that although when considered alone the Coronal Deviation and Forward Sagittal Displacement show some significance in separating the mobility of the SiSt transfers, they provided no 
additional significant information when in the presence of the other identified features in similar populations.

\subsubsection{Sagittal Deviation Classification}

The classification algorithm results were verified by the clinical team's SiSt transfer database, as shown in Table 5.3. The 'Video Output' refers to the video verification performed by the clinical team, and the 'Algorithm Output' refers to the outputs of the sagittal deviation classification. Based on the tested transfers, the algorithm was capable of automatically classifying mobility-impaired transfers with an overall accuracy of $92.1 \%$. The sensitivity resulted in $92.0 \%$ and the specificity in $92.1 \%$, leading to an overall accuracy of $92.1 \%$.

TABLE 5.3: RESULTS OF IMPAIRMENT CLASSIFICATION DURING SIST TRANSFERS

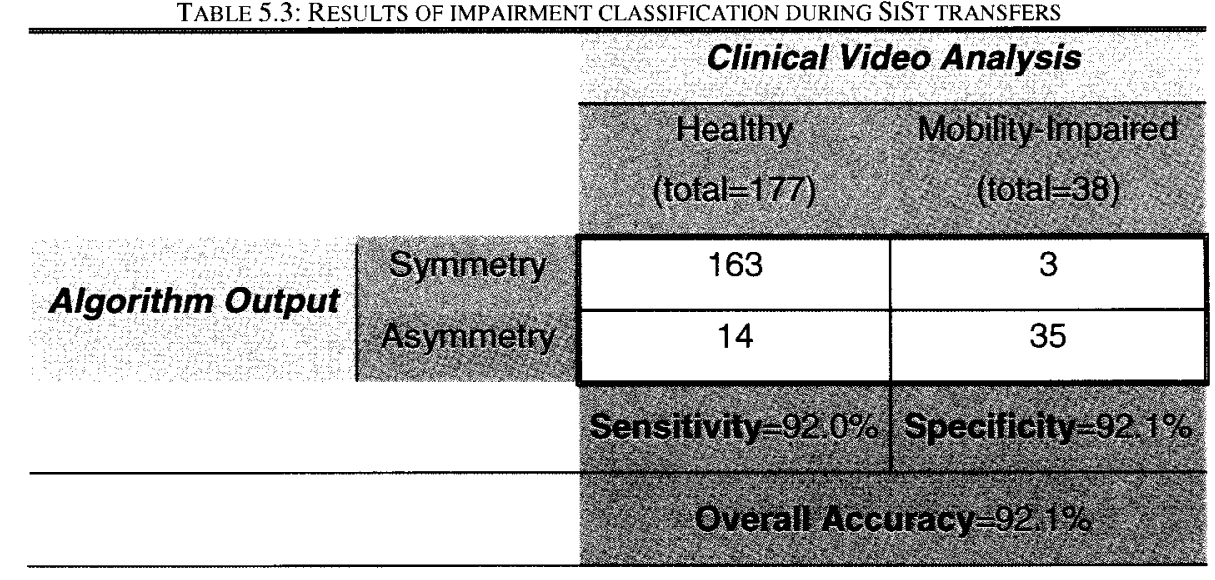

\subsection{Discussion}

The results of this chapter are promising in relation to expected trends and in comparison to recent mobility literature. Further tests with new samples are needed to verify whether the current results can be interpolated to the entire population. New samples should be collected from participants with a wide range of mobility levels to observe finer 
variations in the extracted features. Data from the same patients over longer periods of time would also better validate the ability of the features to capture improvements or degradations in the sagittal trajectory during bed-exit.

The analysis of the CP trajectory does not specifically consider the placement of the hands on the bed as an aid in the SiSt transfer, contrary to the ROI algorithm of Chapter 4. This algorithm enables feature extraction from those who either do not use their hands during bed-exit or those who place them on their thighs. As introduced in Chapter 1, the algorithms in this thesis are designed to enable a monitoring system that is flexible and adaptable to an occupant's individual needs and situational context.

\subsection{Conclusions}

This thesis chapter has provided a feature extraction algorithm for the assessment of mobility during SiSt transfers. Pressure sequences were collected from underneath a bed mattress and were processed to observe the trajectory of the CP on the bed prior to seatdeparture. Several clinical features were identified and extracted from the trajectories of both healthy and mobility-impaired participants. The measure of sagittal deviation was found to be the most significant feature designed and was successfully implemented in the classification of mobility-impaired transfers. The incorporation of this chapter's extracted features within a smart home monitoring framework could provide the automated detection of a decrease in functional mobility of an occupant over time. If caught early, this could instigate appropriate treatment or the usage of transfer aids and therefore prevent harmful falls which could lead to long-term impairment. 


\section{Chapter 6:}

\section{Transfer Duration Measurements from Bed and Floor Pressure Sequences}

\subsection{Introduction}

In a review of the literature on the relevant determinants of the SiSt task, Janssen et al. reported that the duration of the SiSt was a key variable in most of the papers examined [75]. The measurement of this duration is an important goal in mobility research and becomes the focus of this chapter ${ }^{3}$. Section 6.2 begins by introducing the bed and floor pressure sequences that are the basis of this algorithm. Sections 6.3 and 6.4 present the methods of SiSt transfer start and end time detection. Section 6.5 presents the results of the duration measurements and compares them to recently published studies. A discussion of error sources are found in Section 6.6 and chapter conclusions in Section 6.7. Figure 6.1 depicts the focus of this chapter within the monitoring system design.

\footnotetext{
${ }^{3}$ A significant portion of the contents in this chapter has also been published in [16].
} 


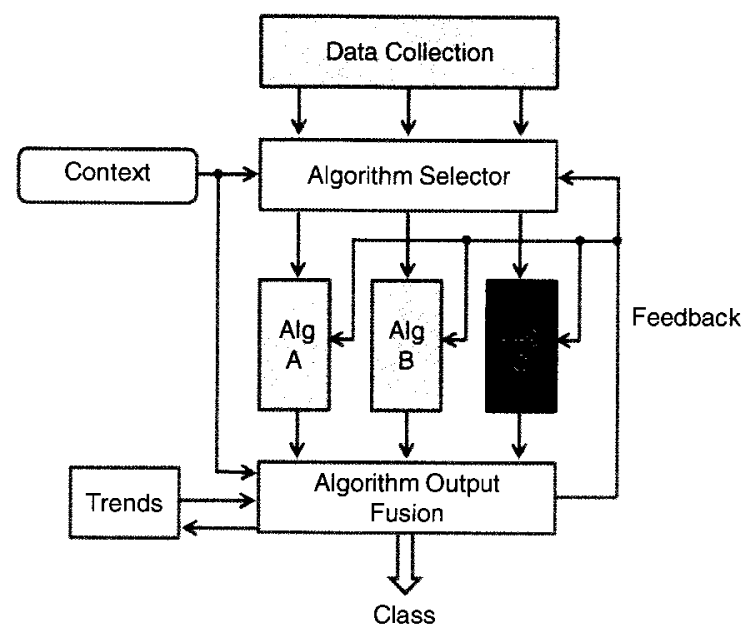

Figure 6.1: Monitoring system feature extraction algorithm C.

In this chapter, the methodology and algorithms are provided to measure the SiSt duration unobtrusively and automatically using pressure-sensitive arrays placed under a bed mattress as well as on the floor. The process is depicted in the system architecture shown in Figure 6.2 and is presented in subsequent sections of this chapter.

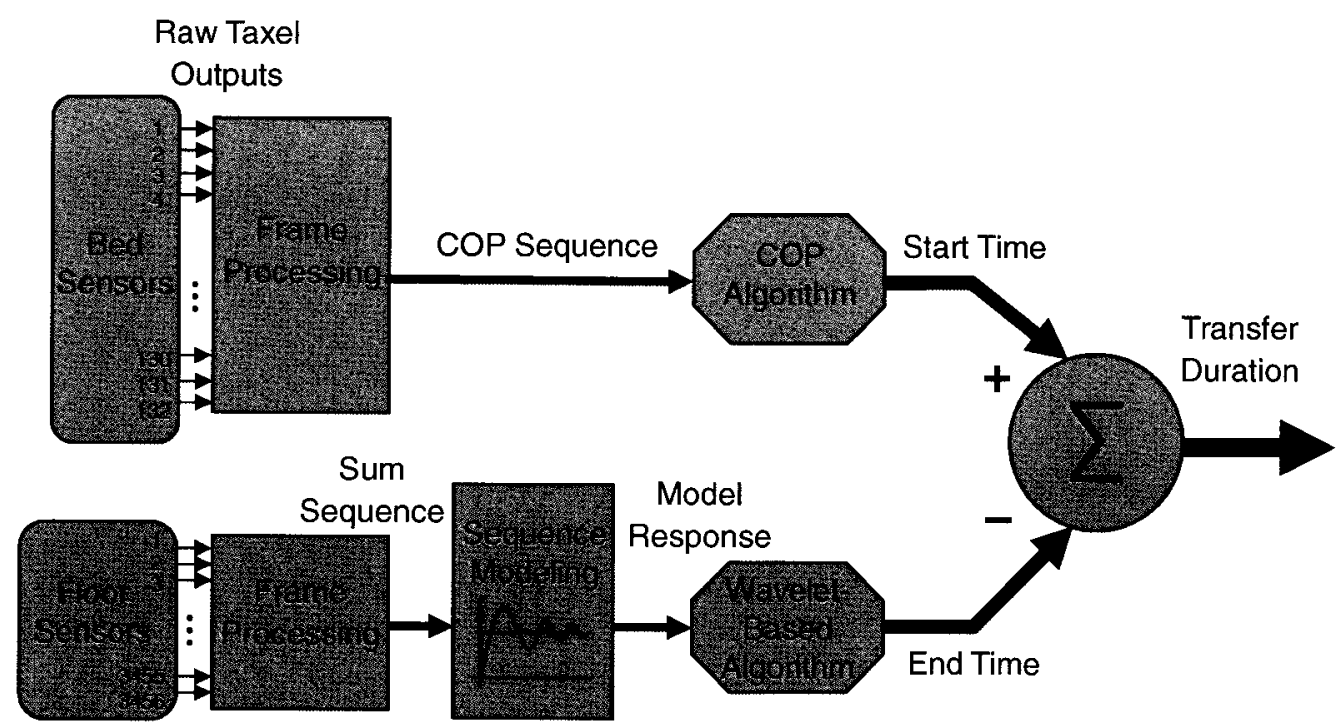

Figure 6.2: System architecture of sit-to-stand duration measurements from bed and floor pressure sequences. 
Although force plates have been used extensively in similar studies, few papers incorporate the pressure exerted by the hips on the sitting surface, which can provide valuable information about the transfer [77]. The unobtrusive nature of the designed techniques avoids patient hesitation and performance bias often attributed to more complex recording equipment involving attachments, installation on the body, and interaction with health professionals [78], [79].

\subsection{Bed and Floor Pressure Sequences}

Figure 6.3 displays the sum of pressure outputs on the bed superimposed by the sum of pressure outputs on the floor for three consecutive trials of a healthy adult performing a lie-sit-stand sequence.

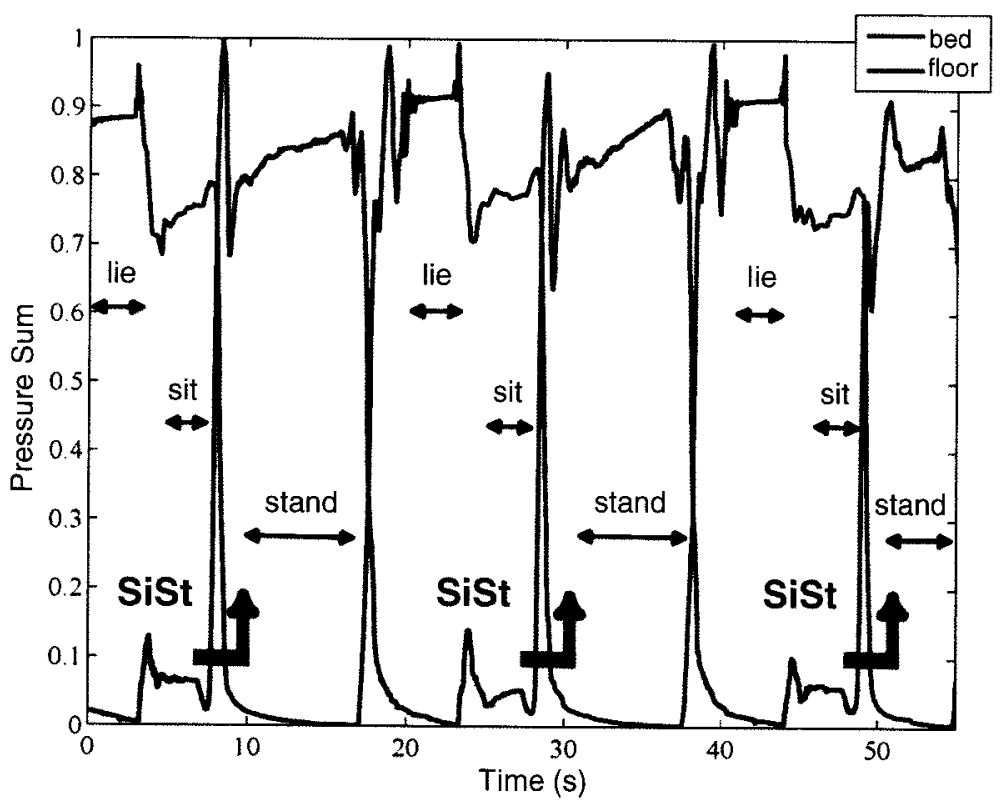

Figure 6.3: Sequences of pressure sums of bed and floor sensors over time.

The bed and floor pressure sequences were aligned in time based on timestamps provided by the Tactex software with each recorded sensor output. The purple arrows indicate the 
occurrence of a SiSt transfer. As expected, these transfers caused a sharp decrease in bed pressure at the same time as a sharp increase in floor pressure.

\subsection{Start of Sit-to-Stand Transfer on the Bed}

The detection of the start of the SiSt is based on the pressure exerted on the bed and the anterior motion of the body during the initial phase of the transfer. The y-coordinate of the center of pressure, $C P_{y}(n)$, is defined along the width of the bed as shown by the axes in Figure 6.4 and as previously introduced in Chapter 5. An increase in $C P_{y}(n)$ signifies a shift of pressure toward the anterior edge of the bed on which the participant is sitting, whereas a decrease signifies a shift back toward the posterior edge. The algorithm detects the time at which the $C P_{y}(n)$ begins to increase, representing the start of the SiSt transfer.

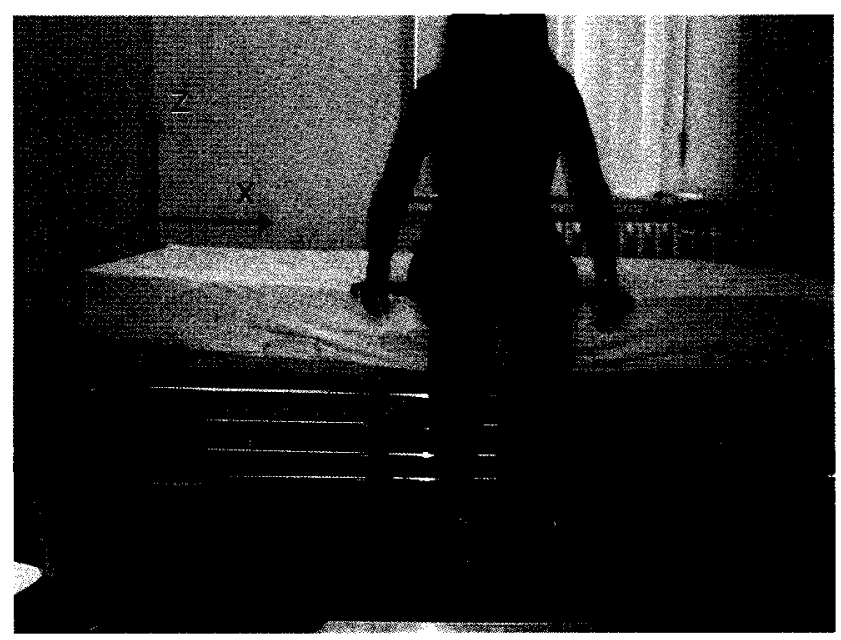

Figure 6.4: Orientation of bed sensor axes in sit-to-stand duration measurements.

\subsubsection{Center of Pressure Sequence}

At each timestamp, the outputs of the bed mats were mapped to form a frame of corresponding sensor locations and pressure outputs. Each frame was then processed to 
extract the location of $C P_{y}(n)$ as in (5.2). To facilitate comparisons across participants of different body weights, the sequences were first normalized to values between zero and one. Figure 6.5 contains a sample of the $C P_{y}(n)$ sequence from five consecutive trials of a healthy participant. The green marker delineates the start of each SiSt transfer extracted from the clinical video analysis.

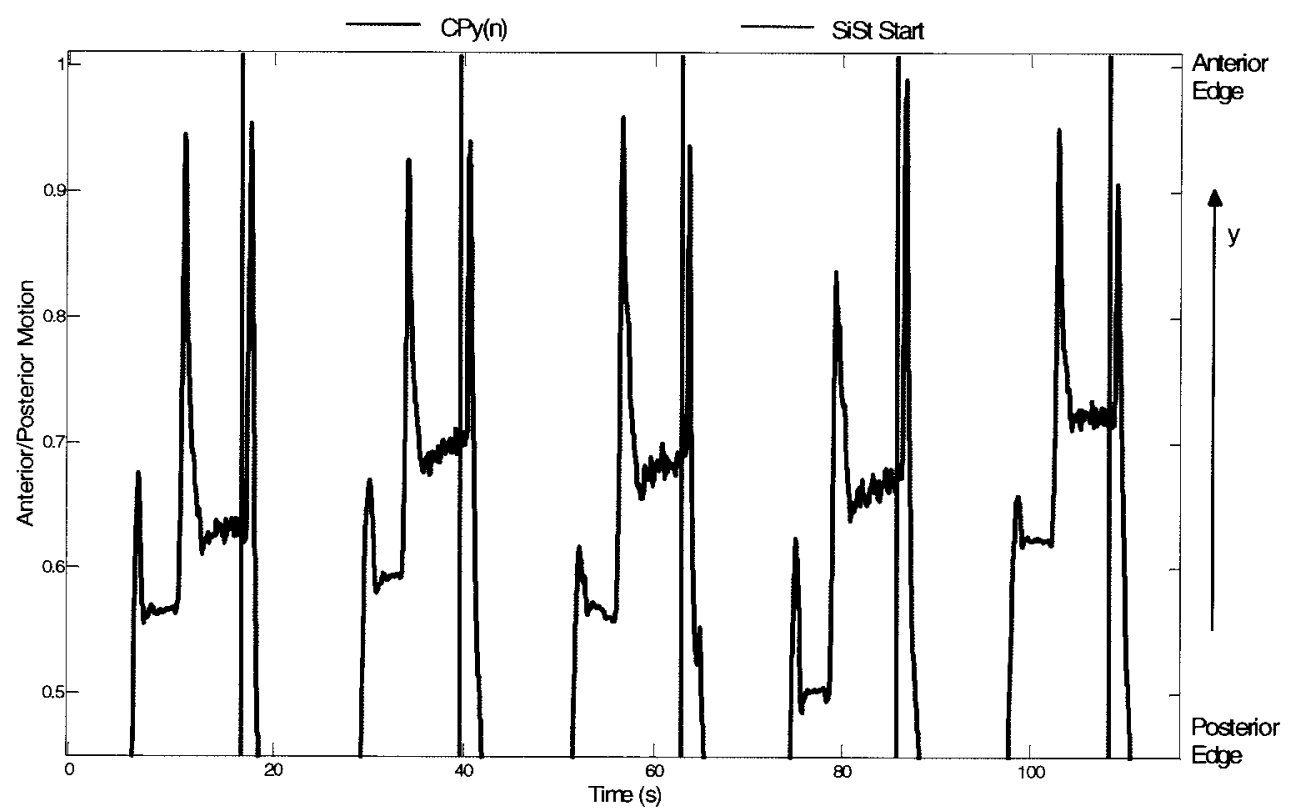

Figure 6.5: $C P_{y}(n)$ sequence for five consecutive SiSt transfers.

\subsubsection{Start Time Detection}

The $C P_{y}(n)$ sequences were manually extracted for each SiSt transfer based on the trial markers defined during data collection. They were upsampled to match the floor plate sampling rate of $50 \mathrm{~Hz}$ and then smoothed by applying a moving-average low-pass filter. This served to reduce the high-frequency noise present in the SiSt transfer and left a picture of the overall forward motion toward the anterior edge of the bed. Based on tests performed on control subjects, a threshold was set to the median of the first $20 \%$ of the 
sequence values, corresponding to the participant sitting upright on the bed. The timestamp of the first sequence value to cross the threshold was considered the starting time of the SiSt. Figure 6.6 displays the start detection of a transfer from the same healthy participant as in Figure 6.5. The solid black marker delineates the starting time based on visual coding of the video record and the solid red marker highlights the start determined by the CP algorithm.

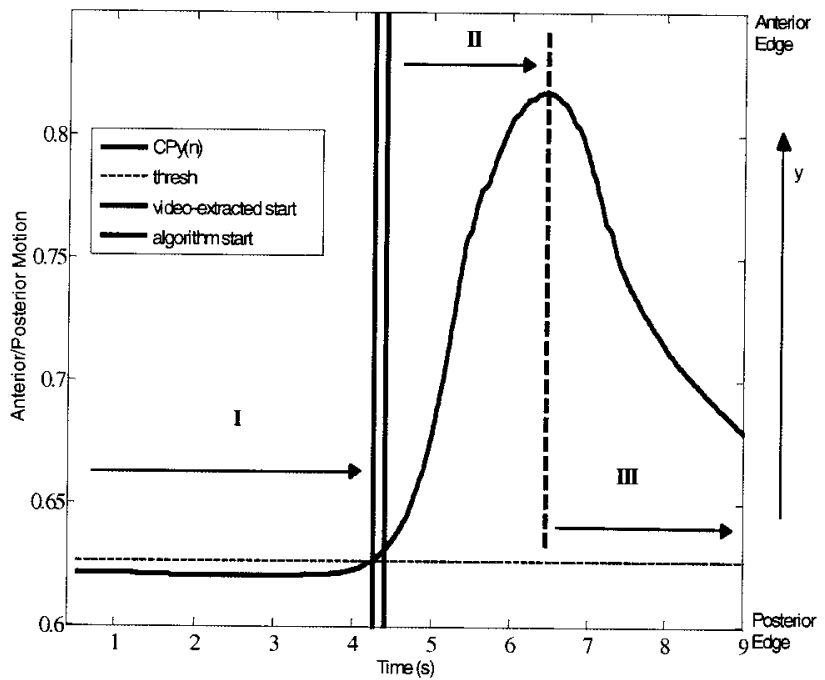

Figure 6.6: Transfer start detection with delineated sections of I: sitting position, II: SiSt transfer, and III: standing position.

\subsection{End of Sit-to-Stand Transfer on the Floor}

\subsubsection{Transfer Modelling}

At each timestamp, the outputs of all floor plate sensors were extracted and summed to form a sequence of total pressure over time. A sample of the pressure sequences of five consecutive SiSt transfers performed by a healthy participant is shown in Figure 6.7. The red and green markers delineate the video-based start and end of the SiSt transfers respectively. 


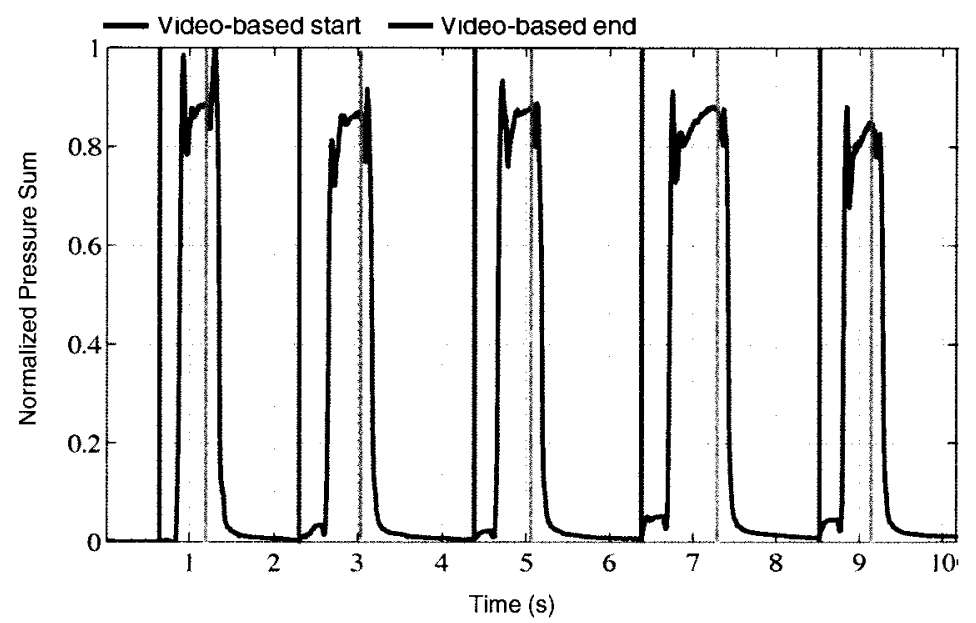

Figure 6.7: Normalized sum of floor pressure for five consecutive SiSt transfers.

It can be seen that there are differences in the SiSt movement within the trials of the same participant and this is true across all participant groups. Differences within healthy participants were due to changes in natural factors such as bed-exit speed or the amount of momentum built by using the hands to push off the bed and on to the floor. Differences within mobility-impaired participants were due to instances of muscle weakness and imbalance issues. While the specific response characteristics can vary greatly from one transfer to the next, the transfers exhibited an overall similarity to the step response of an underdamped system. The transfer began at a low value, where the participant placed minimal pressure on the floor while sitting on the bed. A sharp rise occurred as the participant initially stepped onto the floor plate. The response continued increasing until the participant was fully off the bed and all of their weight was on the floor. This was followed by a period of stabilization in which the participant reached an upright standing position while establishing a "steady-state" balance on their two lower limbs. With the underlying assumption that the transfer of pressures from the bed to the floor plate could 
be approximated by a low-order linear system [80], a third-order underdamped transfer function with integrator was chosen as the most representative model for the floor pressure behaviour. The order was selected as a compromise between what was high enough to produce the required response characteristics such as overshoot and oscillations but low enough to avoid overfitting the data. The continuous-time transfer function in the Laplace domain is given by

$$
H(s)=\frac{K}{s\left(1+2\left(\zeta / \omega_{n}\right) s+\left(s / \omega_{n}\right)^{2}\right)(1+T s)}
$$

where $K$ represents the gain, $T$ the time constant, $\zeta$ the damping ratio, and $\omega_{n}$ the natural frequency of the first and second-order components of the transfer function. The modelled response to an input step is defined as

$$
Y_{e s t}(s)=\frac{H(s)}{s}
$$

Equivalently in the time domain the estimated modelled response results in

$$
y_{e s t}(t)=K \int_{-\infty}^{\infty}\left(1-\frac{e^{-\zeta \omega_{n} \tau}}{A} \sin \left(\omega_{n} A \tau+\phi\right)\right)\left(1-e^{-(t-\tau / T)}\right) d \tau
$$

where $A=\sqrt{\left(1-\zeta^{2}\right)}$ and $\phi=\tan ^{-1}(A / \zeta)$.

All calculations for the determination of the end of the SiSt transfer were done on the resulting modelled transfer response. While the same model was used to estimate all transfers for all participants, the model parameters varied for each according to their individual characteristics. Figure 6.8 depicts a SiSt transfer of a healthy participant superimposed by its model response. 


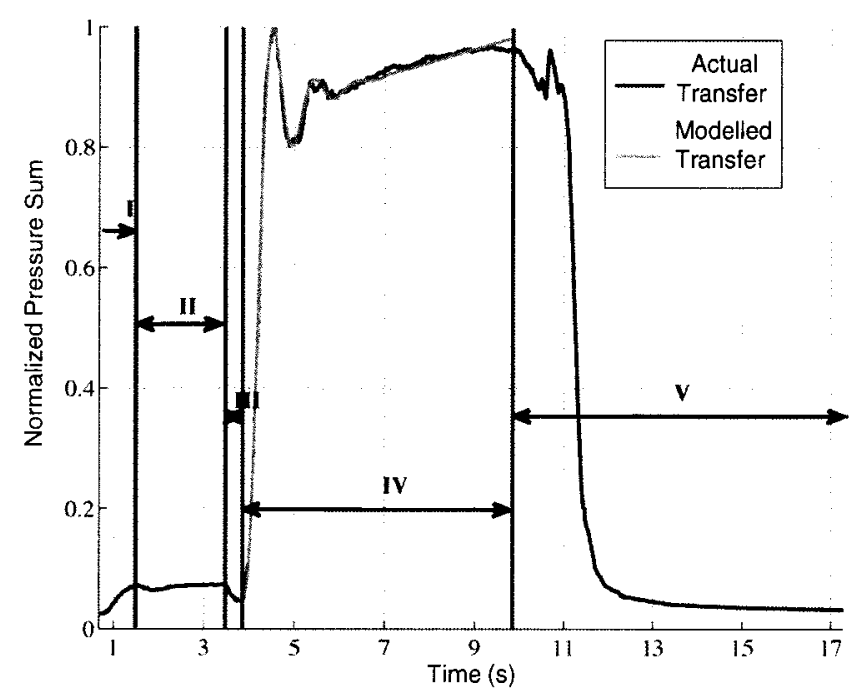

Figure 6.8: Modelled SiSt transfer from floor data with delineated sections of I: end of previous stand-to-sit (StSi) transfer, II: feet resting on floor plate, III: start of SiSt transfer, IV: modelled part of SiSt transfer, and V: StSi transfer.

\subsubsection{End Time Detection}

The detection of the end of the SiSt transfer is a challenging problem since the human body never remains perfectly still when standing. As shown in Figure 6.8 , the pressure variation in the stabilization phase never reaches zero. The objective of end detection in this section is focused on determining the point at which the response reaches a minimum level of variation.

Wavelets are known for being particularly useful in the characterization of biomedical signals [81]. Among the possible families of wavelets, the spline wavelets have many desirable properties such as compactness, symmetry, and regularity [82]. In this chapter, biorthogonal spline wavelets were investigated since they can be conveniently generated with Finite Impulse Response (FIR) filters and are short, symmetrical, and very regular [83]. For each SiSt transfer, the third-order model response 
was obtained as introduced in 6.4.1. Wavelet decomposition was performed and at various levels the signal was reconstructed based on the corresponding detail coefficients. The reconstructed detail signal was expected to characterize the finer variations of the transfer response and, more specifically, show the low variability associated with the beginning of the stable part of the SiSt transfer. After normalizing the reconstructed signal at level $L$, the parts of the signal response falling below a specific threshold $T h$ indicated stable portions. The end of the SiSt transfer was chosen as the first instant corresponding to the longest stable portion.

The choice of wavelet, level of decomposition, and threshold were optimized across all participants and transfers, for all wavelets from one to six vanishing moments, levels of decomposition $L=3$ to $L=8$, and thresholds up to $5 \%$ of the maximum of the reconstructed signal. Based on the optimization, the reverse biorthogonal spline wavelet was chosen with three vanishing moments, $L=6$ and $T h=3.2 \%$. Figure 6.9 includes an example of the end detection for the SiSt transfers of a young healthy participant. The

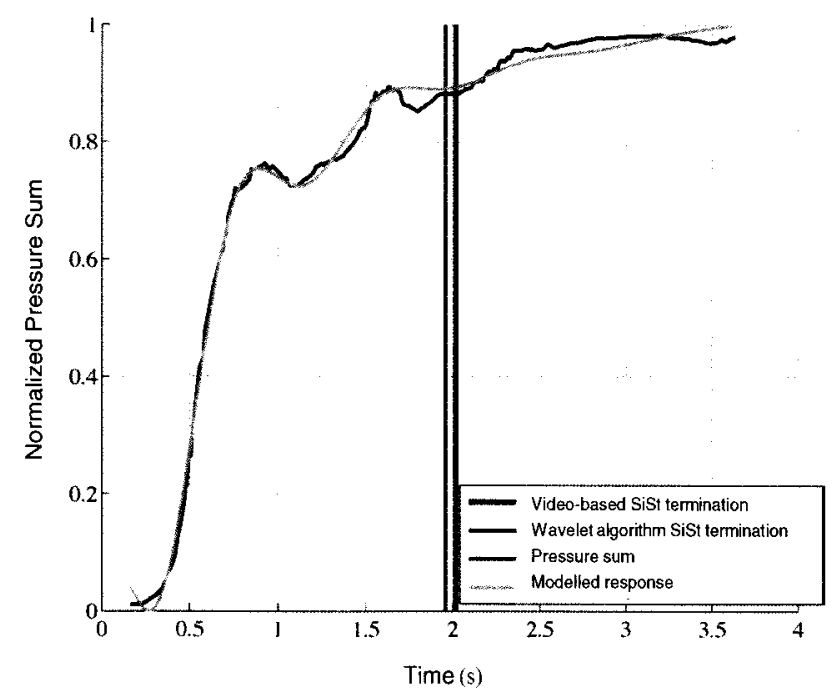

Figure 6.9: Transfer end detection. 
blue line corresponds to the floor plate sequence and the green line to its modelled response. The black vertical line indicates the end of the SiSt transfer based on the clinical video analysis and the red line indicates the algorithm's end time detection.

\subsection{Results and Verification}

\subsubsection{Sit-to-Stand Duration Results}

The mean SiSt transfer durations determined using both the pressure-based algorithms and the video recordings are displayed by participant group in the first two rows of Table 6.1. The subsequent three rows display comparable results published recently based on valuable studies using video processing [37], accelerometers [47], and gyroscopes [48].

TABLE 6.1: VIDEO-BASED (VB) AND PRESSURE-BASED (PB) SIST DURATION TIMES

\begin{tabular}{|c|c|c|c|c|}
\hline & \multicolumn{2}{|c|}{ Healthy } & \multicolumn{2}{|c|}{ Mobility-impaired } \\
\hline & $\begin{array}{l}\text { Young } \\
\text { Healthy }\end{array}$ & Senior Healthy ${ }^{a}$ & Post-stroke $^{b}$ & Post-hip \\
\hline Video-based (s) & $2.13 \pm 0.32$ & $2.93 \pm 0.47$ & $3.84 \pm 1.42$ & $5.52 \pm 1.84$ \\
\hline Pressure-based (s) & $2.31 \pm 0.63$ & $2.88 \pm 1.13$ & $3.57 \pm 1.69$ & $5.35 \pm 2.66$ \\
\hline Goffredo 2009 [37] (s) & $2.31 \pm 0.55$ & $2.41 \pm 0.54$ & & \\
\hline Salarian 2007 [47] (s) & & $2.82 \pm 0.22$ & $3.54 \pm 0.53$ & \\
\hline Najafi 2002 [48] (s) & & $2.90 \pm 0.47$ & $3.73 \pm 1.06$ & \\
\hline \multicolumn{5}{|c|}{$\begin{array}{l}\text { a termed "old adult" in [37], "control elderly" in [47], and "low fall risk" in [48]. } \\
\text { "termed "Parkinson's" in [47], and "high fall risk" in [48]. }\end{array}$} \\
\hline \multicolumn{5}{|c|}{ The SiSt duration extracted from the pressure sequences for the young healthy } \\
\hline \multicolumn{5}{|c|}{ participants $(2.31 \mathrm{~s})$ is identical to that found in Goffredo's "young adult" group [37]. } \\
\hline \multicolumn{5}{|c|}{ While the senior healthy group $(2.88 \mathrm{~s})$ is about $0.4 \mathrm{~s}$ slower than Goffredo's "old adult" } \\
\hline
\end{tabular}


young and senior healthy groups. The pressure-based duration for healthy seniors is very similar to those in both Salarian's "control elderly" group of $2.82 \mathrm{~s}$ [47] and Najafi's "low fall risk" seniors of $2.90 \mathrm{~s}$ [48]. The SiSt for post-stroke participants (3.32 s) is also comparable to the data from individuals with Parkinson's disease reported by Salarian (3.54 s) [47] and Najafi's "high fall risk" seniors (3.73 s) [48].

A Kruskal-Wallis ANOVA test determined significant differences between healthy and mobility-impaired participants $(p<0.001)$. This result is especially valuable for the ongoing monitoring of patients, as the SiSt duration becomes an indicator of overall physical health. The box plot in Figure 6.10 depicts the statistical difference between the two groups.

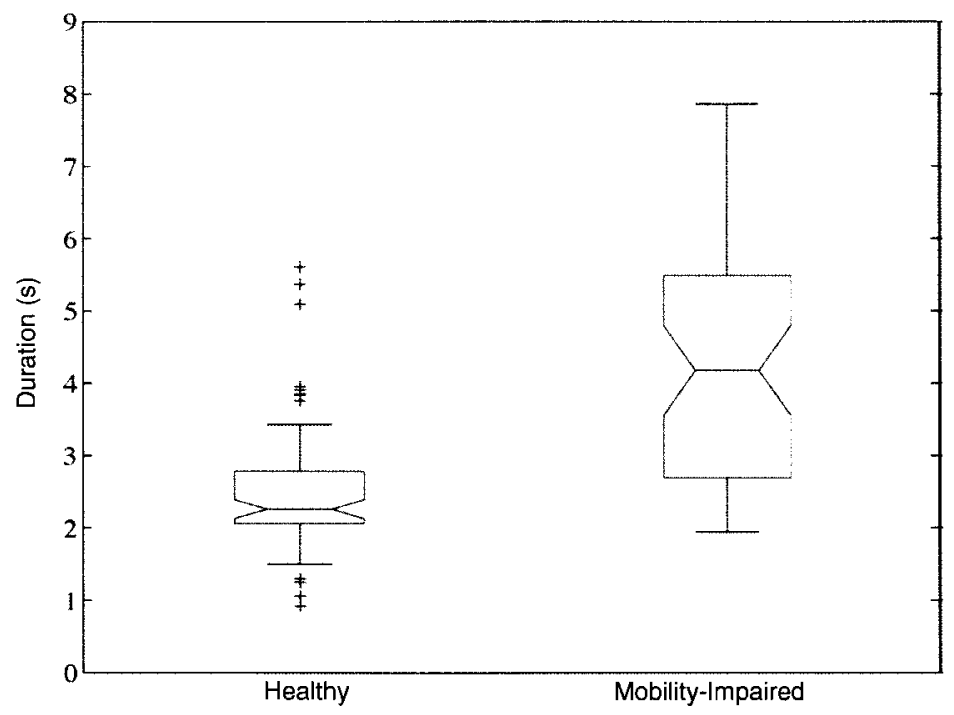

Figure 6.10: Pressure-based SiSt duration medians and deviations for healthy and mobilityimpaired participants.

The red center line of each box denotes the group's median, and the top and bottom edges denote the $25^{\text {th }}$ and $75^{\text {th }}$ percentile of its samples. The notches on the left and right sides 
of each box display the variability of the median between samples of the corresponding group. The lack of any overlap of the notches between the two groups indicates a difference in the medians at a $5 \%$ significance level based on the assumption of the same continuous distribution.

\subsubsection{Duration of Intermediate Phases}

Once the start and end times of the SiSt were determined, additional timing information was extracted from within the SiSt transfer. Figure 6.11 displays the transfer of a healthy participant divided into two phases, $\mathrm{T} 1$ and $\mathrm{T} 2$. $\mathrm{T} 1$ was defined as the time from the start of the transfer to the seat-departure of the participant from the bed. At this timestamp, the normalized pressure on the bed is seen to decrease to a value below 0.10 and the floor pressure is seen to increase to above 0.80 , signifying that the participant has transferred their weight from the bed to the floor. $\mathrm{T} 2$ is defined as the time from the seat-departure point to the end of the SiSt transfer.

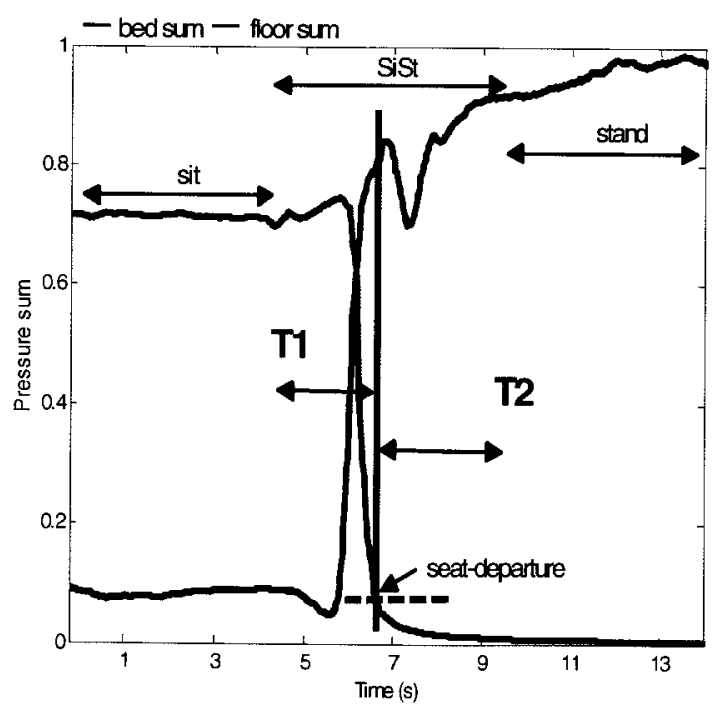

Figure 6.11: Intermediary phases $\mathrm{T} 1$ and $\mathrm{T} 2$ of the SiSt transfer. 
The intermediate phase information was expected to provide further insight into the differences between healthy and mobility-impaired participant transfers. Figure 6.12 contains a scatter plot with the relationship between the T1 and T2 phase durations. It can be seen that the values of $\mathrm{T} 1$ and $\mathrm{T} 2$ for healthy participants clustered together between 0.5 and $2.0 \mathrm{~s}$ whereas the mobility-impaired group covered a much wider range of phase times between 1.0 and $5.0 \mathrm{~s}$.

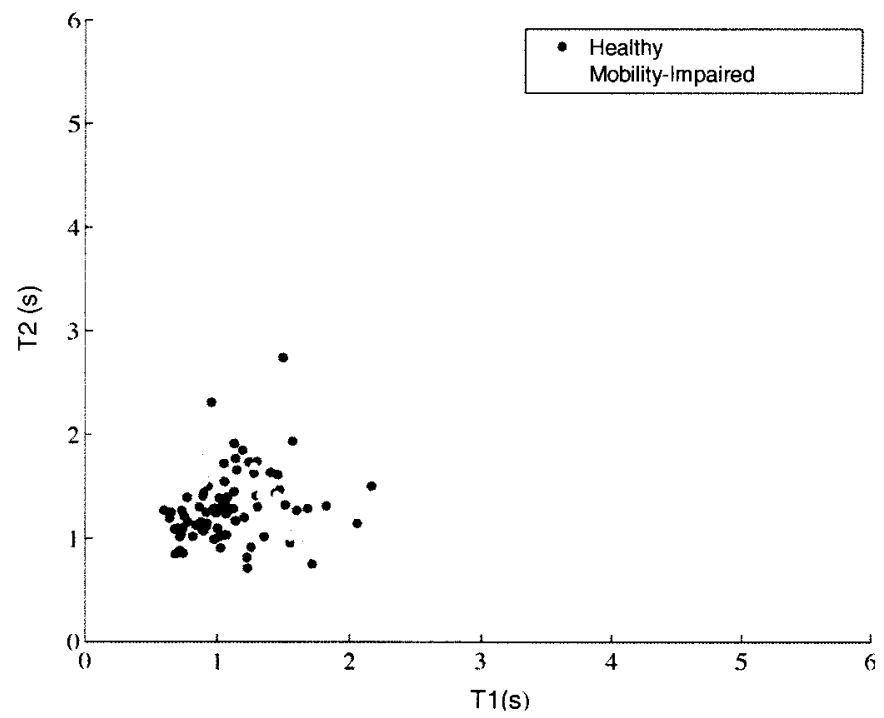

Figure 6.12: Scatter plot of SiSt phase T1 versus T2 for healthy and mobility-impaired participants.

\subsubsection{Start and End Time Errors}

The error in the SiSt start time estimation was defined as the difference between the time detected by the automated start algorithm and the clinical start time extracted visually from the video recordings. The error in the SiSt start time estimation averaged around $0.29 \mathrm{~s}$ for healthy participants and around $0.49 \mathrm{~s}$ for the mobility-impaired participants. The error in the SiSt end time was defined as the difference between the time detected by the automated end algorithm and the clinical end time extracted from the video 
recordings. The error in the end times typically averaged around $0.52 \mathrm{~s}$ for healthy participants and $0.77 \mathrm{~s}$ for the mobility-impaired group.

\subsection{Discussion}

As verified by the clinical video analysis, the durations of SiSt transfers were successfully measured unobtrusively from pressure measurement sequences. Errors in the start time were typically due to the pressure variance on the bed mat during the sitting phase immediately preceding the SiSt transfer. If a participant moved about significantly while sitting, this was sometimes mistaken by the algorithm as the beginning of the transfer. Similarly, errors in the SiSt end time were largely due to a lack of stability of participants in the standing position proceeding the transfer. While healthy participants were almost always stable on their feet, mobility-impaired participants often did not reach a point of complete stability. Some transfers also resulted in a poor fit to the thirdorder model, with no overshoot or no apparent periodic oscillations.

The error in estimation of both SiSt start and end times was also partially due to the difficulty in determining the exact control times visually from the video recordings. The video contributed the largest source of precision error. At a sampling rate of $30 \mathrm{frames} / \mathrm{s}$, each frame covered a length of $0.033 \mathrm{~s}$ and produced a minimum precision error of $\pm 0.017 \mathrm{~s}$ for start and end times. Differences within participant groups also contributed to the error as participants within one group sometimes displayed widely varying SiSt characteristics. 


\subsection{Conclusions}

This chapter has presented an algorithm for the measurement of SiSt transfer durations unobtrusively using pressure-sensitive arrays. Valuable information can be obtained from pressure mats placed under the mattress of a bed with a pressure-sensitive floor plate located next to the bed on the floor. While the pressure exerted on the floor has been previously measured in the study of SiSt motion, the methods in this chapter combine the measure with the pressure exerted on the mattress in the initial phase of the transfer.

The forward motion of the $\mathrm{CP}$ along the bed provides a valuable indicator of the start time of the SiSt transfer. The step response of a third-order transfer function can be used to model the foot pressure during the progression of the transfer to a stable standing position. The resulting durations measured from both pressure sequences and video data across the different participant groups are comparable to those found in recent clinical publications. While there appears to be no significant difference in SiSt durations between younger adults and healthy seniors, the difference between healthy and mobilityimpaired participants was found to be significant. As mentioned in previous chapters, while these results are promising, additional tests with new samples are needed to verify whether they can be interpolated to the entire population. Within a smart home environment, monitoring the SiSt duration of an occupant over time could provide information as to their level of functional mobility. Increasingly longer durations could indicate a loss of coordination and increased hesitancy during bed-exits which can signify more serious underlying conditions. 


\section{Chapter 7:}

\section{Context in Mobility Monitoring}

\subsection{Introduction}

This thesis chapter presents the contextual information considered in the smart home monitoring system. Section 7.2 begins by introducing the three sources of context in the current design. Section 7.3 then presents the algorithms and results obtained by extracting sensed context features from pressure-sensitive grab bars. The chapter ends with conclusions in Section 7.4. Figure 7.1 depicts the focus of this chapter within the monitoring system design.

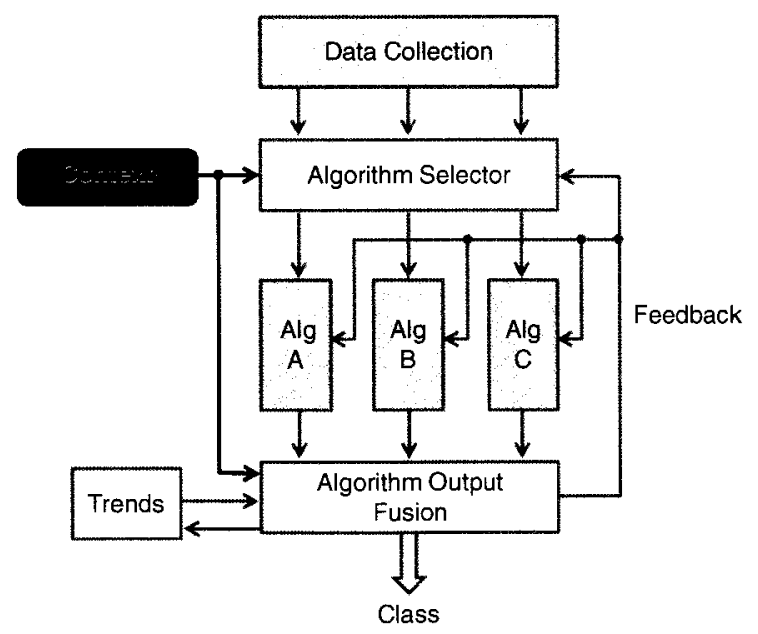

Figure 7.1: Monitoring system context-awareness. 


\subsection{Sources of Context-A wareness}

In this thesis, context-awareness is incorporated in order to improve the assessment of the occupant's mobility. Context-awareness can be viewed from different perspectives depending on the objectives and applications [84]. Henricksen et al. defined three principle sources of context information: (1) sensed: collected by physical and logical sensors; (2) human: entered by users or administrators of a system; and (3) derived: from other types or combinations of information sources [85], [86]. This thesis incorporates the three sources of context into the system design as they relate to the environment of a smart home occupant. The next three sections describe each type in further detail.

\subsubsection{Human Context - Manual Information Entry}

While the goal of smart environments is to provide automated monitoring, it is still useful to enable the option of manually entered information to allow user-specific concerns to be addressed [87], [88]. In the current monitoring system, activity information could be obtained manually by caregivers, family members or in-vivo checkups made to the home. This could be in the form of notes input to a computer by a nurse to annotate a particular behaviour, by a family member who records the date and time of a particular visit, or by the occupant entering their own personal impressions or health concerns.

\subsubsection{Derived Context - Electronic Health Records}

In 2001, Health Canada launched an organization called Canada Health Infoway, with the purpose of establishing a pan-Canadian standardized Electronic Health Record (EHR) system [89]. The organization covers all provinces and territories working together to 
coordinate and manage information in response to the increased incidence of chronic diseases, a shortage of general practitioners, and a shift from acute to home care [90]. The EHR can be used as an input into a context-aware monitoring system, providing information related to the patient's prescribed medications, diagnosed conditions and general medical history.

\subsubsection{Sensed Context - Pressure-Sensitive Grab Bars}

The lavatory is a common place for falls to occur, especially during transfers to and from the bathtub or toilet. To aid in these transfers, the Canadian Standards Association recommends the use of grab bars affixed to the wall or in the form of toilet commodes [91]. In this chapter the usage of grab bars by an occupant is analyzed as an indicator of their status of functional mobility. Incorporated into a context-aware monitoring system, the indicators of deteriorating mobility captured by the bed mats could be verified by those captured by the grab bars in the lavatory.

\subsection{Sensed Context Using Pressure-Sensitive Grab Bar Sequences}

This section presents the incorporation of sensed context using pressure-sensitive grab bars. Section 7.3 .1 begins by introducing the sensor calibration followed by the contact location and force estimation. Section 7.3.2 describes the significant features defined by the TAFETA clinical team and the corresponding feature extraction. Section 7.3.3 presents the results and verification of grab bar usage based on data from clinical trials ${ }^{4}$.

\footnotetext{
${ }^{4}$ Significant portions of the contents in this section have also been published in either [19] or [21].
} 


\subsubsection{Sensor Calibration and Output Estimation}

\section{Calibration Procedure}

The grab bars of a standard-sized commode were instrumented by Kinotex pressure sensors as introduced in Section 3.5.1. To calibrate the sensor outputs to the forces applied, it was necessary to relate these output levels to known input forces. The minimum and saturation values of each sensor were first determined and the outputs were normalized to values between zero and one. An estimate for zero force was collected by recording the output with no weights on the bar. Weights of $1 \mathrm{~kg}, 2.5 \mathrm{~kg}$ and $5 \mathrm{~kg}$ were then suspended by a ribbon of $1 \mathrm{~cm}$ in width at the locations directly above each sensor. Their outputs were recorded for $15 \mathrm{~s}$ each at a sampling rate of $10 \mathrm{~Hz}$, resulting in 150 samples per location. Baseline testing indicated that the last 50 samples contained the least amount of noise due to the manual movement of the weight and were therefore considered the most accurate measure of the applied force at each location. Figure 7.2 depicts the resulting grab bar installation with a suspended calibration weight.

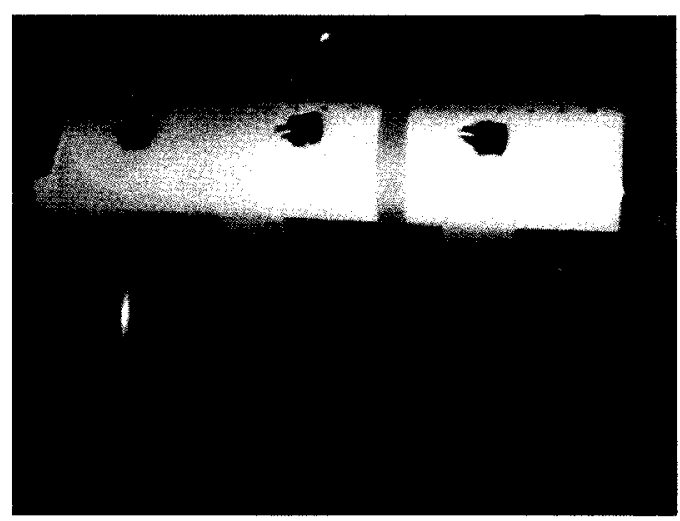

Figure 7.2: Calibration of embedded pressure sensors. 
The calibration outputs were used to perform a least squares fit to a second order polynomial describing the sensor behaviour with increasing force. The result of this fit for each of the three sensors is shown in Figure 7.3.

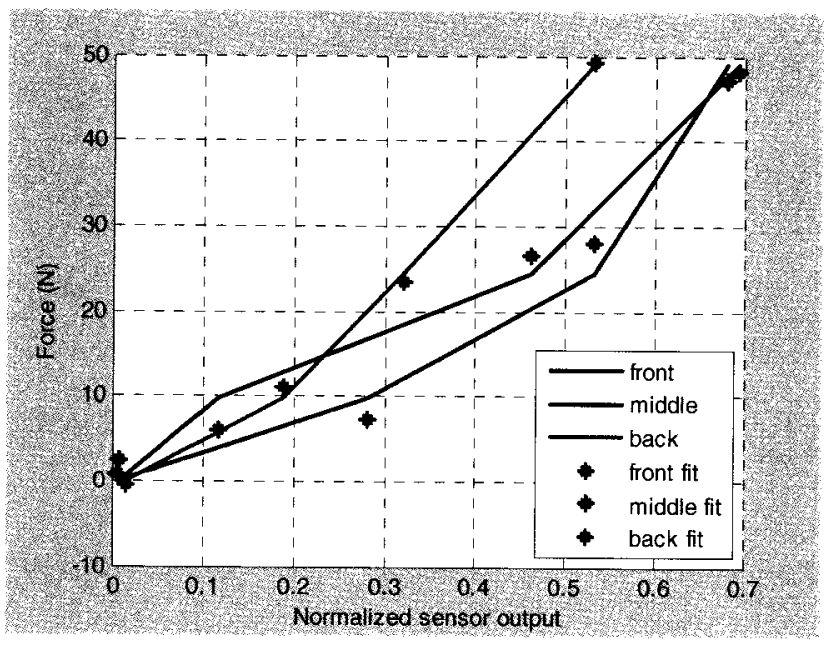

Figure 7.3: $2^{\text {nd }}$-order model fit of force as a function of sensor output.

\section{Location and Force Estimates}

When a force was applied at any point along the bar, the three sensors each responded with a corresponding output voltage reflecting their measured pressure. The location and force were determined using the artificial neural network-based algorithms published in [19] and [20] respectively. Figure 7.4(a) contains the three sensor outputs recorded from a trial of stand-to-sit ( $\mathrm{StSi}$ ) and SiSt transfers performed by a young participant. Figure 7.4(b) and Figure 7.4(c) contain the resulting sequences of location and force estimates. At around $2.5 \mathrm{~s}$, the three sensors were activated as the participant used the grab bar to sit down. After about one additional second the pressure was released and remained off while the participant was seated. At around $8.5 \mathrm{~s}$ the sensors were activated once again as the participant returned to a standing position. 
(a)

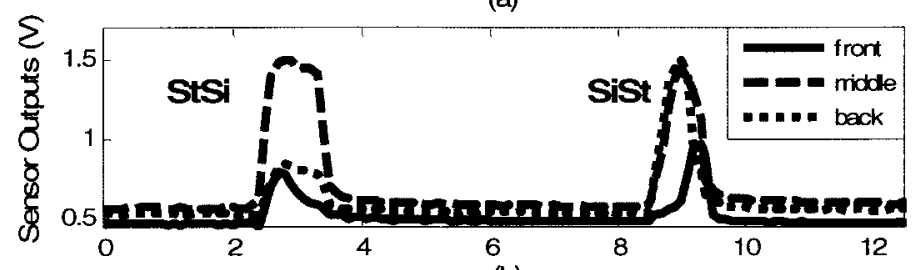

(b)

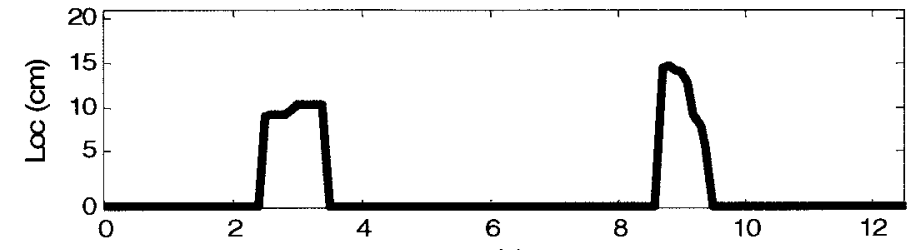

(c)

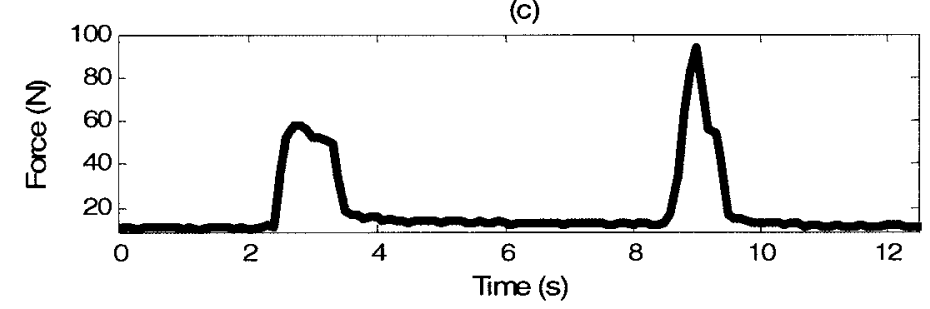

Figure 7.4: Data sequence of (a) sensor outputs, (b) location estimates and (c) force estimates for StSi and SiSt transfers performed by a young participant.

The contribution of each sensor output in Figure 7.4(a) can be seen in the estimates of Figure 7.4(b) and Figure 7.4(c). During the StSi transfer, the middle sensor was considerably higher than that of both the anterior and posterior ends. This is reflected in the location estimate at that time being just under $10 \mathrm{~cm}$, corresponding to the user applying force on the middle of the $21 \mathrm{~cm}$ grab bar. A picture of the bar with the direction of increasing location is found in Figure 7.5. Throughout the StSi transfer the back sensor remained fairly constant whereas the front sensor began to decrease slightly, causing the contact location estimate to increase to just over $10 \mathrm{~cm}$ by the end of the sit. This represents the participant releasing their hand in a forward sliding motion once seated. The force estimates of Figure 7.4(c) also followed these loading and unloading patterns closely. During the SiSt the middle and back sensor outputs began equally high 
resulting in a location estimate of about $14 \mathrm{~cm}$, which is the middle point between these two sensor locations. Towards the end of the SiSt the front sensor quickly increased its contribution, resulting in a quick decrease in estimated contact location. The location estimates conformed to expectations as the StSi transfer is accompanied by a backward motion limited by the backrest of the seat, whereas the SiSt transfer was accompanied by a forward motion with no limitations. The force estimates during the SiSt reflected a shorter duration of applied force than during the $\mathrm{StSi}$. This behaviour was also expected since one must release their grip on the bar to reach a full standing position.

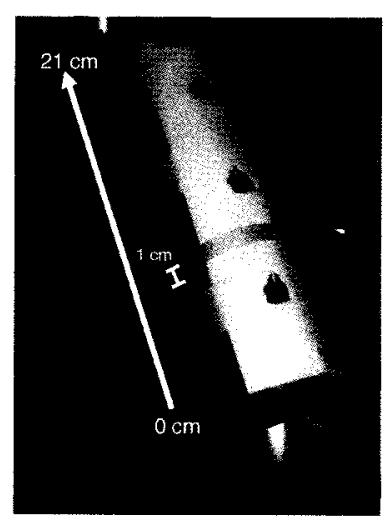

Figure 7.5: Direction of increasing location along the grab bar.

\subsubsection{Grab Bar Sequences and Feature Extraction}

\section{Clinical Feature Definition}

In collaboration with the TAFETA clinical team, several features of interest were chosen to describe the loading pattern on each commode grab bar during weight transfers from both SiSt and StSi:

1) Transfer time: duration of time over which the grab bars are used by the participant for weight transfer 
2) Maximum position: location of applied force which is closest to the most posterior position of the commode

3) Time to maximum position: time taken to reach the most posterior position

4) Position range: absolute distance along the bar covered by the applied force during the transfer

5) Maximum force: maximum force exerted on the bar

6) Time to maximum force: time taken to reach the maximum force exerted on the bar

7) Force range: absolute difference between maximum and minimum forces applied to the bar during the transfer

\section{Extracted Features}

A sample of location and force sequences collected from a senior participant are displayed in Figure 7.6.

(a)

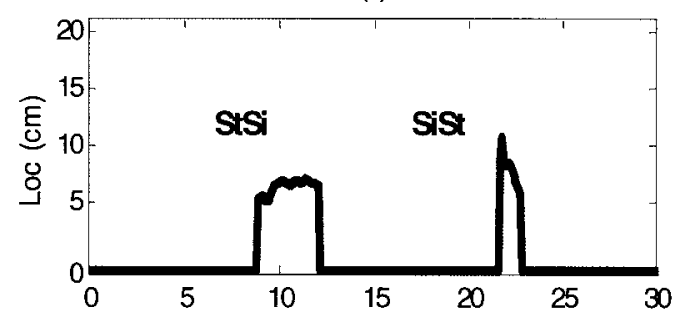

(b)

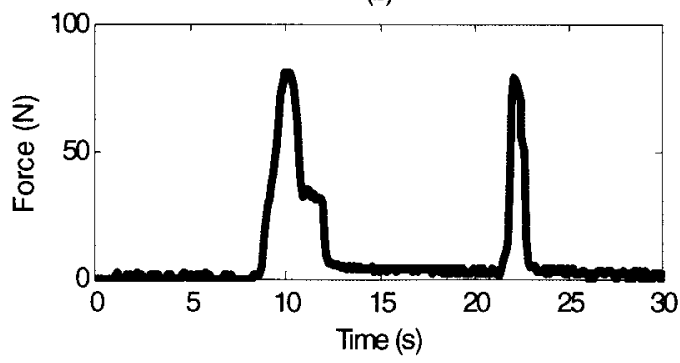

Figure 7.6: Data sequence of (a) location estimates and (b) force estimates for StSi and SiSt transfers performed by a senior participant. 
While the overall pattern is similar to the younger sample in Figure 7.4, differences can be seen in transfer times as well as in maximum location and applied forces. To define the start and end of the transfers, a lower threshold of $10 \mathrm{~N}$ was set on the force output past which the action was considered to have begun. This threshold was selected based on baseline noise tests performed during calibration. The clinical features defined previously in this chapter were extracted from the location and force sequences by an automated algorithm. A sample of the feature extraction for the StSi transfer of the sequence is depicted in Figure 7.7, while the corresponding values are included in Table 7.1.

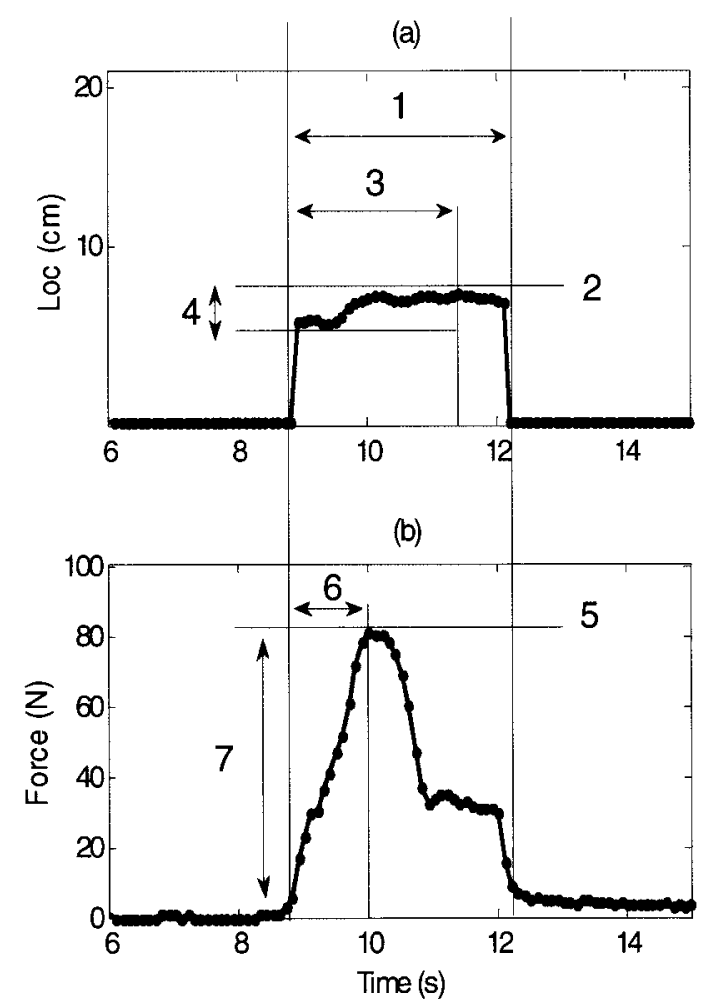

Figure 7.7: Feature extraction from the sequences of (a) location estimates and (b) force estimates during the StSi transfer of a senior participant. 
TABLE 7.1: CLINICAL FEATURES EXTRACTED FROM THE STSI TRANSFER OF A SENIOR PARTICIPANT

\begin{tabular}{cll}
\hline \hline Number & Feature Name & Value \\
\hline $\mathbf{1}$ & Grab Time (s) & 3.4 \\
\hline $\mathbf{2}$ & Maximum Position (cm) & 7.1 \\
\hline $\mathbf{3}$ & Time to Maximum Position (s) & 2.6 \\
\hline $\mathbf{4}$ & Position Range (cm) & 1.9 \\
\hline $\mathbf{5}$ & Maximum Force (N) & 81.0 \\
\hline $\mathbf{6}$ & Time to Maximum Force (s) & 1.2 \\
\hline $\mathbf{7}$ & Force Range (N) & 75.2 \\
\hline
\end{tabular}

\subsubsection{Grab Bar Usage Results and Verification}

The clinical features were extracted for both the StSi and SiSt trials of all participants. The average StSi grab time for the young participants (1.24 s) compared well to the average StSi time for the healthy group (1.2 s) measured using accelerometers in [46]. Seniors generally produced longer grab times for both the StSi (1.81 s) and SiSt (1.35 s) transfers than the young participants. Seniors also generally exerted less force on the bars. The younger participants tended to make contact with the bar at a position closer to the posterior edge of the commode. While the position range was larger in seniors than in young participants during the StSi transfer, the reverse was true during the SiSt.

\subsection{Conclusions}

In this chapter, three sources of context have been identified for incorporation into the smart home monitoring system: human, derived and sensed. A method of sensed context was designed to automatically extract clinical features regarding the usage of grab bars during transfers on and off of a toilet commode. This provided additional information with respect to the mobility of an occupant during weight transfers and could therefore be 
used in the verification of the features extracted from the bed mat algorithms. The unobtrusive nature of this work contributes a useful tool which can be incorporated in the monitoring system to detect the decline in strength or mobility of a smart home occupant over time. 


\section{Chapter 8:}

\section{Context-Aware Smart Home Monitoring}

\subsection{Introduction}

This chapter presents the incorporation of context-awareness into the smart home monitoring system design 5 . In future work, context-awareness can be incorporated through an inference engine as part of an expert clinical decision support system, however this is out of the scope of the current thesis. In this chapter, context information is manually input to a simulated context-aware environment using the features extracted from the actual clinical trials. The chapter begins by introducing the simulated environment in Section 8.2. Section 8.3 presents simulated scenarios of mobility classification based on the three algorithms of Chapters 4,5 and 6 as well as the context provided by the pressure-sensitive grab bars introduced in Chapter 7. Section 8.4 presents the incorporation of the algorithm selector and context-aware feedback loop. The chapter ends with conclusions in Section 8.5. Figure 8.1 depicts the focus of this chapter within the monitoring system design.

\footnotetext{
${ }^{5}$ A significant portion of the contents in this chapter has also been published in [23].
} 


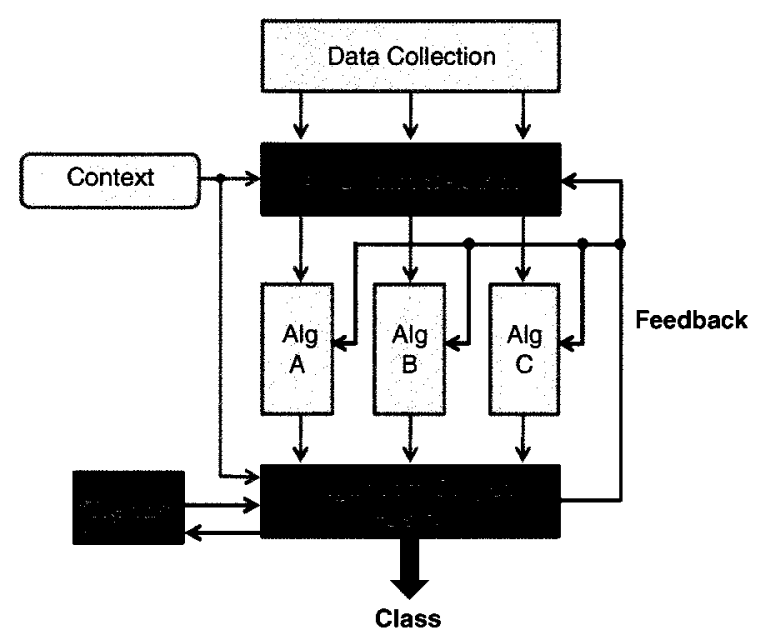

Figure 8.1: Monitoring system algorithm selector, trends, algorithm output fusion and feedback loop.

\subsection{Simulated Context-Aware Environment}

The three context sources introduced in Chapter 7: human, derived and sensed, were incorporated into a simulated context-aware environment. The sensed context in the design was provided by the grab bar outputs in the lavatory of the smart home. Since in reality this data was collected from different participants than the bed data, specific results were selected that were representative of the behaviour of participants with the same mobility levels. The human context, which was designed to be input by a caregiver, visitor or occupant of the smart home, was simulated based on expected scenarios provided by the TAFETA clinical team. The derived context was designed to be automatically input from a patient's electronic health records. In practice, access to these records is highly restricted due to ethical and logistical considerations. Health information of the clinical trial participants was therefore only used as a source of context 
when provided voluntarily in the medical history questionnaires completed during data collection.

\subsection{Classification of Occupant Mobility Based on Feature Extraction Algorithms and Context Provided by Pressure-Sensitive Grab Bars}

This section combines the results of the feature extraction algorithms with the sensed context provided by the pressure-sensitive grab bars. The focus here is on demonstrating how, as the occupant progresses through their day, the sensed context could provide further insight into their mobility status and produce a warning to a caregiver should further attention be required.

The function of the context-aware monitoring system design is shown through the classification of two simulated scenarios: (1) the occupant in the normal range of their daily behaviour, summarized in Figure 8.2, and (2) the occupant experiencing abnormal behaviour, summarized in Figure 8.6. In this section there is no previous knowledge about the status of the, therefore the algorithm selector considers all algorithms equally. The first two columns of each figure contain the algorithm label and sequence features considered in the given scenario. These are followed by the expected outputs of the same features, derived from the occupant's recent history. The actual output column contains the results of the feature extraction algorithms introduced in Chapters 4, 5 and 6. The algorithm output fusion column presents an initial warning of 'OK', 'Warning' or 'High Warning' based on the extracted features. The final column contains the decision output by a simulated context-aware classifier based on the algorithm output fusion from both the bed mats and the pressure-sensitive grab bars. 


\begin{tabular}{|c|c|c|c|c|c|}
\hline $\begin{array}{c}\text { Scenario } \\
1\end{array}$ & $\begin{array}{l}\text { Sequence } \\
\text { reature }\end{array}$ & $\begin{array}{c}\text { Expected } \\
\text { Output }\end{array}$ & $\begin{array}{l}\text { Actual } \\
\text { Otutput }\end{array}$ & $\begin{array}{c}\text { Algorilhm } \\
\text { Oluput } \\
\text { Fusion }\end{array}$ & $\begin{array}{l}\text { Classifier } \\
\text { Output }\end{array}$ \\
\hline $\begin{array}{l}\text { Alg A } \\
\text { ROI }\end{array}$ & Hand usage & both & $\mathbf{R H}$ & \multirow{3}{*}{ Warning } & \multirow{7}{*}{ Normal } \\
\hline Alg B & $\begin{array}{l}\text { Sagitial } \\
\text { deviation }\end{array}$ & $0.43 \mathrm{~cm}$ & $0.41 \mathrm{~cm}$ & & \\
\hline $\begin{array}{l}\text { Alg C } \\
\text { Duration }\end{array}$ & Duration & $2.2 \mathrm{~s}$ & $2.8 \mathrm{~s}$ & & \\
\hline \multirow{4}{*}{$\begin{array}{l}\text { Grab Bar } \\
\text { Context }\end{array}$} & Hand usage & both & both & \multirow{4}{*}{ OK } & \\
\hline & Crab time & $0.6 \mathrm{~s}$ & $0.6 \mathrm{~s}$ & & \\
\hline & $\begin{array}{l}\text { Position } \\
\text { range }\end{array}$ & $3.0 \mathrm{~cm}$ & $3.2 \mathrm{~cm}$ & & \\
\hline & $\begin{array}{l}\text { Maximum } \\
\text { force }\end{array}$ & $65 \mathrm{~N}$ & $68 \mathrm{~N}$ & & \\
\hline
\end{tabular}

Figure 8.2: Simulated Scenario 1: occupant is classified as being within normal behaviour range.

\subsubsection{Scenario 1: Normal Behaviour}

The first row of Figure 8.2 shows that the scenario began with a bed-exit during which the ROI algorithm was expected to recognize both the left and right hand throughout the transfer sequence. The expected normalized pressure sequences for the three regions are shown in Figure 8.3(a). The actual scenario produced the pressure curves in Figure 8.3(b), where it is shown that only the right hand was used for the SiSt transfer.

The second row of Figure 8.2 shows that the center of pressure sequence was expected to output a healthy level of $0.43 \mathrm{~cm}$ of sagittal deviation. The expected trajectory towards the front edge of the bed is depicted in Figure 8.4(a) and the actual trajectory, with a sagittal deviation of $0.41 \mathrm{~cm}$, in Figure $8.4(\mathrm{~b})$. The total SiSt duration, as listed on the third row of Figure 8.2, was expected to be measured by the timing algorithm as $2.2 \mathrm{~s}$. The actual duration measured $2.8 \mathrm{~s}$, which was $0.6 \mathrm{~s}$ higher than predicted. The combined deviations between expected and actual outputs of the ROI and 
timing algorithms, highlighted in bold in Figure 8.2, led the algorithm output fusion to flag a level of 'Warning'.

(a) Expected Pressure Sequences of three detected region: Hips, LH, RH, normalized by maximum region pressure

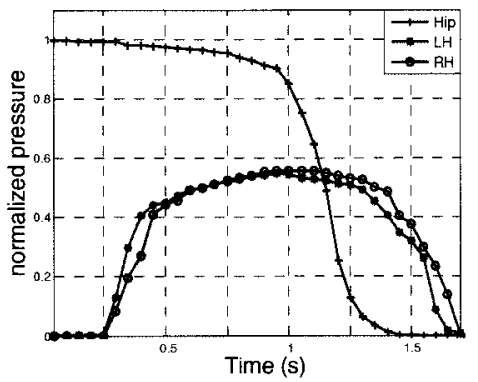

(b) Actual Pressure Sequences of two detected regions: Hips, $\mathrm{RH}$, normalized by maximum region pressure

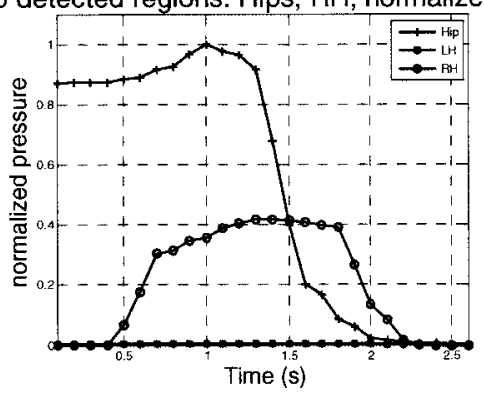

Figure 8.3: Algorithm A - ROI results: normalized pressure sequences during the occupant's SiSt transfers.
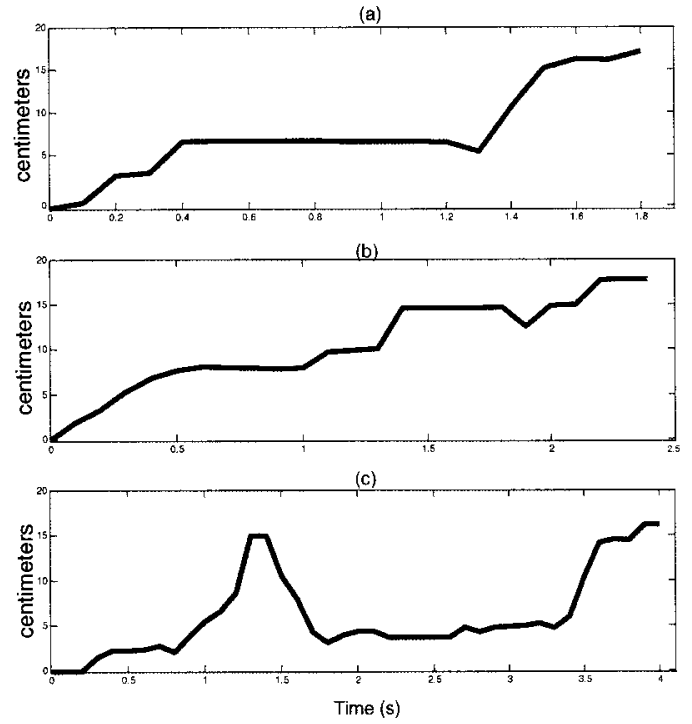

Figure 8.4: Algorithm B - CP results: center of pressure sequences during the occupant's SiSt transfers displaying (a) expected output (b) actual output for Scenario 1 and (c) actual output for Scenario 2. 
After the bed-exit, the occupant was assumed to proceed to the lavatory, where their next transfer was from stand-to-sit onto the toilet commode. The expected context introduced by the pressure-sensitive grab bars, as shown in Figure 8.2, was that both hands would be used to lower the body onto the seat. The occupant was expected to exert forces on average of $65 \mathrm{~N}$ for about $0.6 \mathrm{~s}$ during the stand-to-sit transfer and these forces were to be applied within a range of $3.0 \mathrm{~cm}$ along the length of the bar. As shown in Figure 8.5, the actual outputs in this case were similar to the expected values of $68 \mathrm{~N}$, $0.6 \mathrm{~s}$ and $3.2 \mathrm{~cm}$. Based on the progression of the outputs from the bedroom to the lavatory, the context-aware classification output continued to stay at a normal level. All intermediary outputs would be saved for future use, both to contribute to the expected values of future decisions, as well as to keep track of potential warning signs.
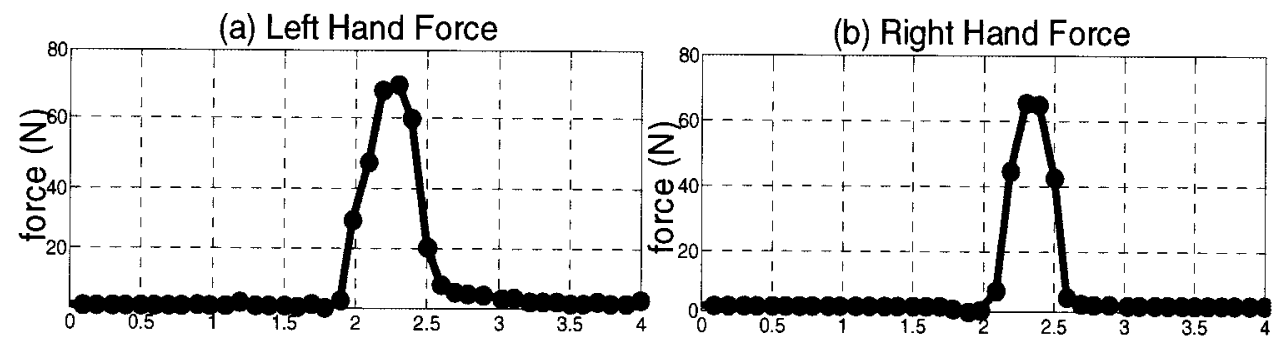

(c) Left Hand Location
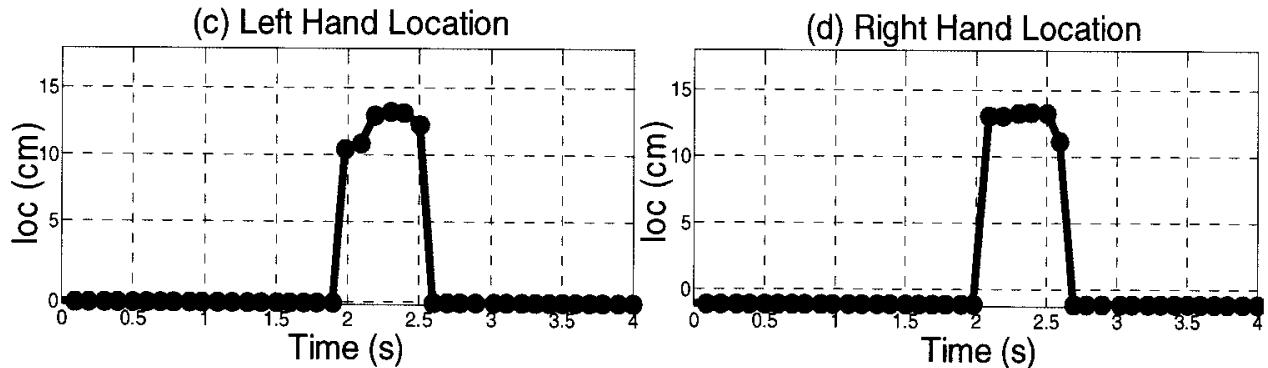

Figure 8.5: Sensed context - pressure-sensitive grab bar results: actual sequences output in Scenario 1: (a) left hand force (b) right hand force (c) left hand location and (d) right hand location. 


\subsubsection{Scenario 2: Abnormal Behaviour}

As shown in Figure 8.6, the second scenario began with a bed-exit in which the ROI algorithm determined that only the right hand was used in the SiSt transfer from the bed, even though the use of both hands was expected. This time the CP trajectory displayed a high deviation of $1.42 \mathrm{~cm}$ by the occupant, depicted in Figure 8.4(c). The total duration of the SiSt transfer was measured at $4.6 \mathrm{~s}$, more than double its expected value of $2.2 \mathrm{~s}$. The combination of these three outputs produced a 'High Warning' level from the algorithm output fusion.

\begin{tabular}{|c|c|c|c|c|c|}
\hline $\begin{array}{c}\text { Scenario } \\
2\end{array}$ & $\begin{array}{l}\text { Sequence } \\
\text { reature }\end{array}$ & $\begin{array}{l}\text { Expected } \\
\text { Output }\end{array}$ & $\begin{array}{l}\text { Actual } \\
\text { Output }\end{array}$ & $\begin{array}{c}\text { Algoritlum } \\
\text { Output } \\
\text { Fusiont }\end{array}$ & $\begin{array}{l}\text { Classifier } \\
\text { Output }\end{array}$ \\
\hline $\begin{array}{l}\text { Alg A } \\
\text { ROI }\end{array}$ & Hand usage & both & RH & \multirow{3}{*}{$\begin{array}{l}\text { High } \\
\text { Warning }\end{array}$} & \multirow{8}{*}{ Abnormal } \\
\hline $\begin{array}{l}\text { Alg B } \\
\text { CP }\end{array}$ & $\begin{array}{l}\text { Sagittal } \\
\text { deviation }\end{array}$ & $0.43 \mathrm{~cm}$ & $1.42 \mathrm{~cm}$ & & \\
\hline $\begin{array}{l}\text { Alg } \mathbf{C} \\
\text { Duration }\end{array}$ & Duration & $2.2 \mathrm{~s}$ & $4.6 \mathrm{~s}$ & & \\
\hline \multicolumn{5}{|c|}{ Pressure-sensitive Grab Bar Context: } & \\
\hline \multirow{4}{*}{ Features } & Hand usage & both & RH & \multirow{4}{*}{$\begin{array}{l}\text { Figh } \\
\text { Warning }\end{array}$} & \\
\hline & Grab time & $0.6 \mathrm{~s}$ & $1.1 \mathrm{~s}$ & & \\
\hline & $\begin{array}{l}\begin{array}{l}\text { Position } \\
\text { range }\end{array} \\
\end{array}$ & $3.0 \mathrm{~cm}$ & $9.0 \mathrm{~cm}$ & & \\
\hline & $\begin{array}{l}\text { Maximum } \\
\text { force }\end{array}$ & $65 \mathrm{~N}$ & $83 \mathrm{~N}$ & & \\
\hline
\end{tabular}

Figure 8.6: Simulated Scenario 2: occupant is classified as experiencing abnormal behaviour and requires further attention.

The abnormal behaviour persisted through to the lavatory, where the pressuresensitive grab bars revealed that only the right hand was used. Depicted in Figure 8.7, the force exerted on the right bar was now $83 \mathrm{~N}$ with a duration of more than $1.1 \mathrm{~s}$ and a range of $9.0 \mathrm{~cm}$. The algorithm output fusion also considered the grab bar results to be 
cause for a level of 'High Warning' and, when combined with the bed results, the classifier output changed from normal to abnormal. At this stage the monitoring system sent notification to a caregiver that further attention was required.

(a) Left Hand Force

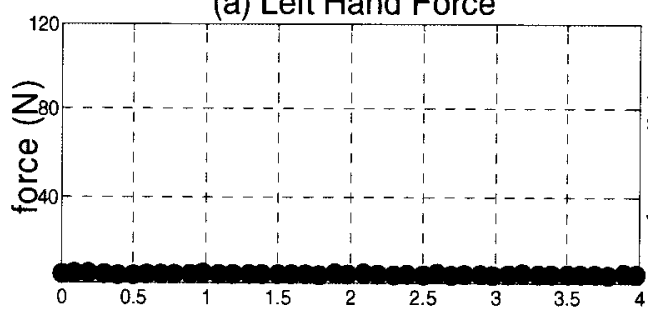

(c) Left Hand Location

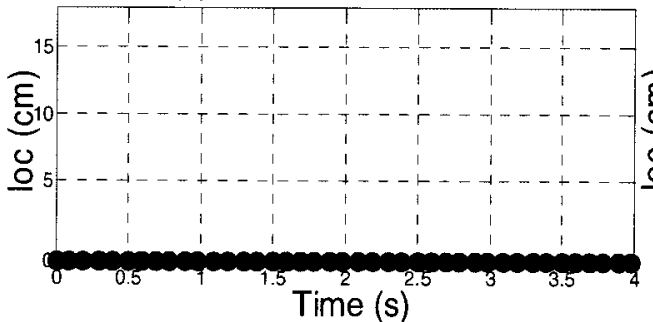

Time (s) (b) Right Hand Force

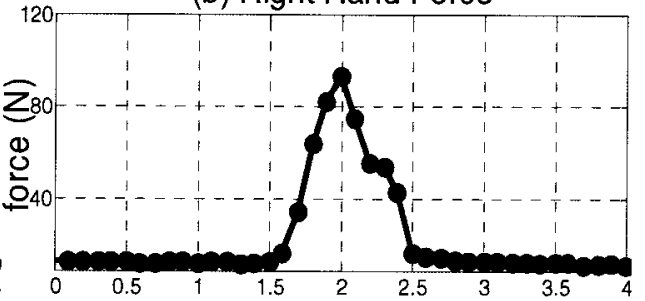

(d) Right Hand Location

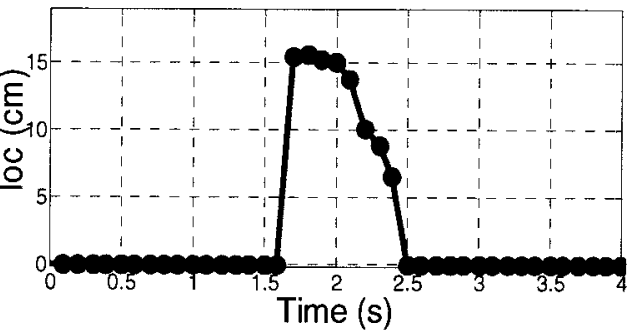

Figure 8.7: Sensed context - pressure-sensitive grab bar results: actual sequences output in Scenario 2: (a) left hand force (b) right hand force (c) left hand location and (d) right hand location.

\subsection{Classification of Occupant Mobility Using Algorithm Selection and Context-Aware Feedback}

\subsubsection{Context-Aware Algorithm Selection}

The feature extraction algorithms presented in this thesis were designed to analyze different aspects of the SiSt transfer while maintaining flexibility and adaptability to a smart home occupant's environment. Depending on the situational context, certain algorithms will be more applicable and others will be rendered irrelevant. For this reason, an algorithm selector was included in the system design to choose the appropriate feature 
extraction methods that will give the most accurate representation of the occupant's mobility status.

In the case of a loss of feeling in one side of the body, such as an occupant who has suffered a severe stroke, the ROI-based feature extraction algorithm may no longer be relevant. If their EHR indicates that they no longer have use of their right hand, the symmetry and right hand usage features become irrelevant while others increase in significance. Similarly, an occupant with higher mobility who does not use their hands or places them on their thighs during the SiSt transfer may require the same considerations. In these cases, the features such as sagittal deviation and SiSt duration could be selected as the most representative measures to be monitored over time.

The algorithm selector also allows flexibility in the instrumentation of the smart home. While the experiments in this thesis have been performed with pressure sensing mats containing many sensors, the algorithms can be easily modified for the implementation of different configurations or fewer sensors. In the case of a row of sensors at the edge of the bed, the algorithm selector may remove the CP-based algorithm as it is no longer applicable. In the case of a small number of sensors, the algorithm may select the duration-based algorithm alone.

Finally, the monitoring system allows the flexibility for physicians and caregivers to monitor specific features of interest to them. Should the supervising physician decide, for example, that they want to specifically monitor the initial position of the occupant on the bed when they attempt to stand, they can manually select this feature from the CP-based algorithm. This could be of particular interest to occupational therapists, who give 
specific suggestions to seniors about how to best perform transfers for increased safety within the home.

\subsubsection{Context-Aware Feedback Samples}

The simulated samples in this section display the function and benefits of the feedback loop in the context-aware monitoring system. It is assumed in these cases that the algorithm selector has been manually set by a physician to consider the ROI algorithm only, as the physicians were specifically interested in the progression of the regions over time. Each sample begins by demonstrating the confounding effects that a change in context can have on the ROI processing. They then show how the context-aware feedback from the algorithm fusion back to the ROI can allow the modification of the region parameters and lead to a corrected classification output.

\section{Human Context}

The regional pressure sequences of a healthy senior's SiSt transfer are shown in Figure 8.8(a) and Figure 8.8(b). Unusual for a healthy participant, a high right hand pressure was maintained throughout the full SiSt transfer. Assuming that a family member was also present at the time of the transfer, this could have been due to a second person sitting on the bed. Manual entries into the visitor's $\log$ could be input to the system as a form of human context and fed back to the feature extraction algorithms. The regional parameters could then be automatically modified and the pressure which appeared as a right hand would be eliminated from consideration in the second pass through the system. The resulting minimal hand usage is shown in Figure 8.8(c). 


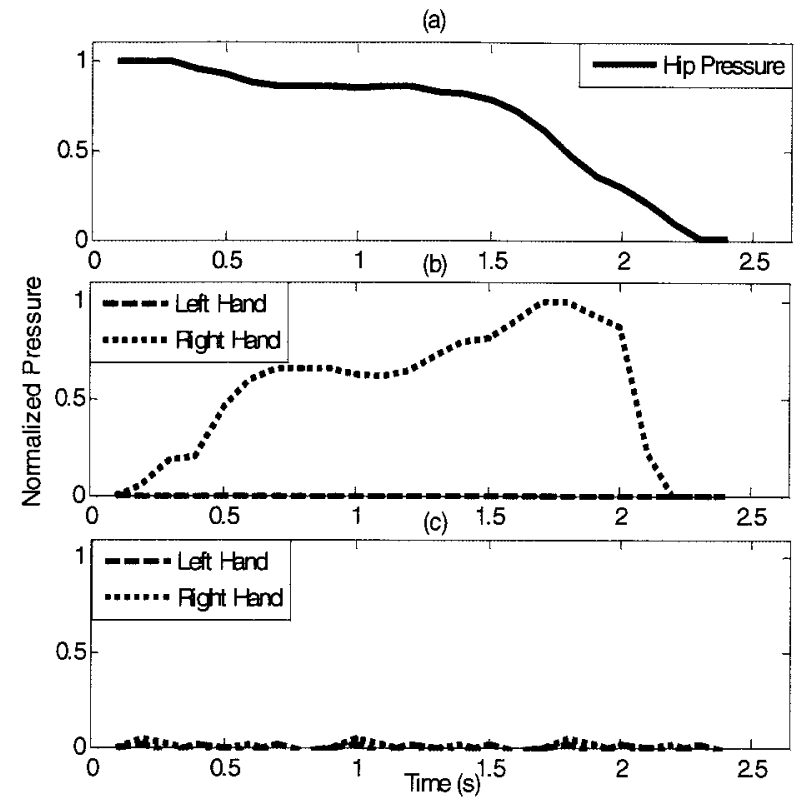

Figure 8.8: Pressure sequences from a healthy senior's SiSt transfer initially misclassified by the ROI algorithm as asymmetrical.

\section{Derived Context}

In Figure 8.9(a) and Figure 8.9(b), the pressure sequences from the SiSt transfer of a post-stroke participant are shown. The ROI algorithm initially output the transfer as having used both hands. A review of their patient history showed that their stroke had caused them to permanently lose the use of their right hand, making it impossible to have the right hand pressure sequence shown. Assuming that this information could be automatically extracted from the electronic health records, this could be input to the system as a form of derived context and fed back to the ROI algorithm. A change in the regional parameters would have then demonstrated that in order to maintain balance during the transfer, the patient moved their hips in a horizontally with greater sway than usual. This caused the appearance of a right hand region in the initial pass through the 
system but could be tracked as part of the hip region in the second. The final result, displaying a left-weighted transfer with some bouncing, is shown in Figure 8.9(c).
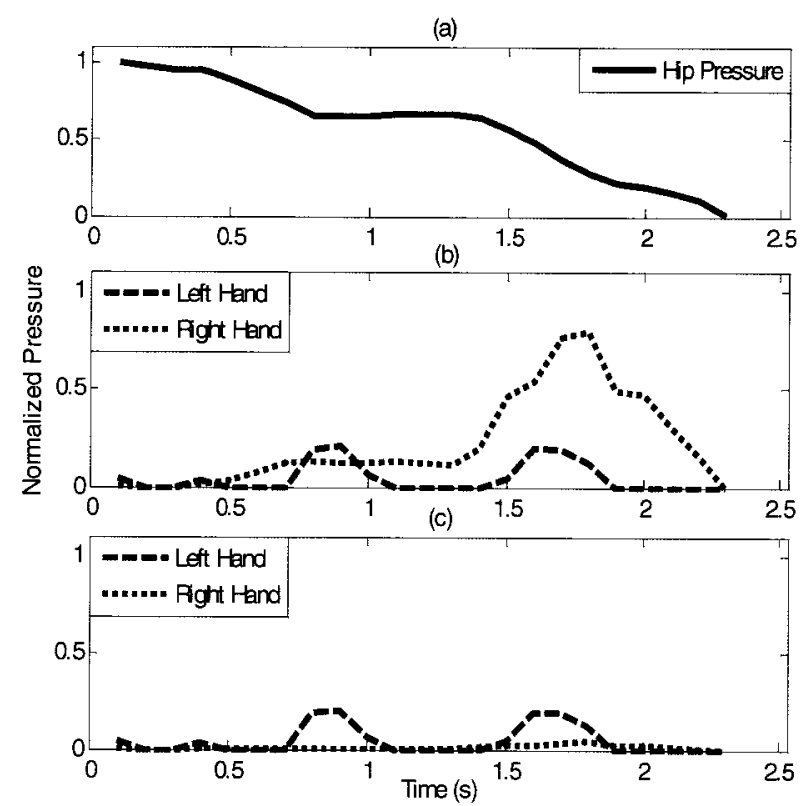

Figure 8.9: Pressure sequences from a post-stroke patient's SiSt transfer initially output as having used both hands.

\section{Sensed Context}

Figure 8.10(a) and Figure 8.10(b) display the pressure sequences resulting from the SiSt transfer of a post-stroke participant, which the ROI algorithm initially classified as being symmetrical. Assuming that the pressure-sensitive grab bars output a high right hand usage immediately after the bed-exit, this could be input to the system as sensed context and the result could be reconsidered. A review of the hip parameters would then have shown that the right hand had been placed slightly underneath the hips, causing much of its force to be confused for the hip region. 

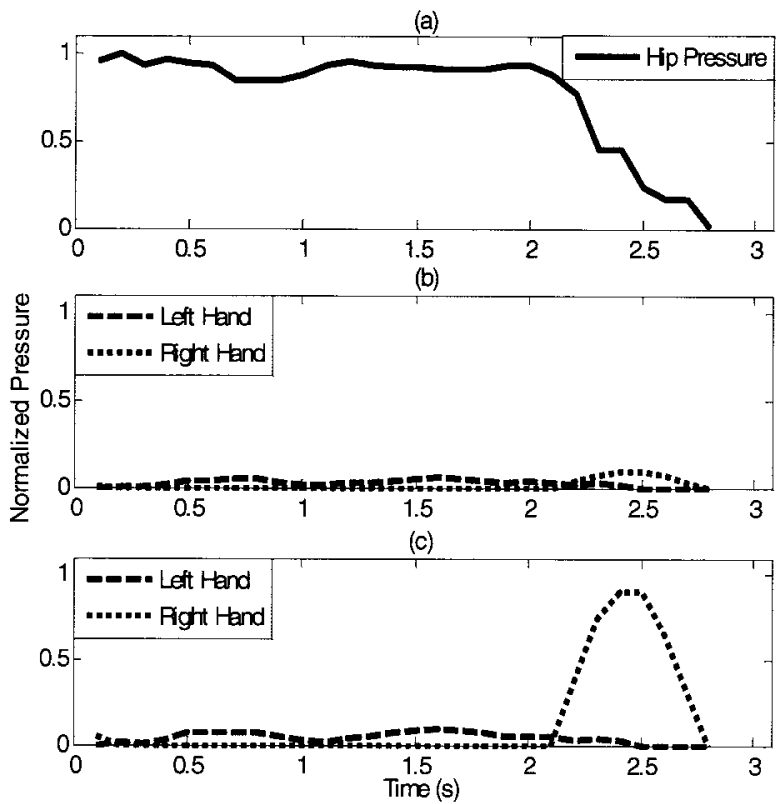

Figure 8.10: Pressure sequences from a post-stroke patient's SiSt transfer initially misclassified by the ROI algorithm as symmetrical.

\subsection{Conclusions}

A simulated context-aware environment was created to observe the function of contextawareness in the mobility monitoring of a smart home occupant. Context-awareness was first simulated by combining the information extracted from the separate rooms of bedroom and lavatory along with the occupant's expected behaviour to produce a representative warning of the occupant's mobility status. The incorporation of the algorithm selector was then presented and the effects of the context-aware feedback were observed through samples which were initially misclassified by the ROI algorithm but subsequently corrected. The context-aware system presented in this thesis can be customized for a particular occupant and can also be integrated within a larger monitoring system composed of different types of sensors or additional sources perceiving context throughout the smart home. 


\section{Chapter 9:}

\section{Conclusions}

\subsection{Thesis Conclusions}

Due to the unprecedented growth in the senior population and to the resulting shortages in financial and personnel resources, there is a growing demand for smart home technology that can contribute to senior care. This thesis has presented a design for context-aware smart home monitoring through the analysis of pressure sequences. The design provides unobtrusive monitoring of the functional mobility of seniors within the smart home environment. The early detection of a decrease in mobility could lead to the prevention of falls and fractures within the home, which can have very hazardous effects on the senior population. The SiSt transfer has been widely used in the assessment of functional mobility and has become the focus of analysis in the system design.

Pressure sensing was used as the primary source of data collection given its flexible and unobtrusive nature. Sensors were placed under the bed mattress, on the floor beside the bed and in grab bars to collect information regarding the occupant's mobility while performing weight transfers throughout the smart home. Data was first collected unobtrusively in the rehabilitation ward of a hospital to observe changes in the mobility 
of patients over time and to concretize the system design considerations. Clinical trials were then performed to collect data from young adults as well as from both healthy and mobility-impaired seniors in a controlled environment. Testing was performed on the various participant groups with the purpose of simulating a smart home occupant who over time would experience a decrease in functional mobility due to underlying conditions. While these trials were not exhaustive clinical grade assessments, they allowed the observation and preliminary verification of clinical features which characterized SiSt transfers in participants of varying mobility levels.

Several algorithms were designed to extract features from recorded pressure sequences and to characterize the differences between healthy and unhealthy mobility. The first algorithm detected the regions of interest during SiSt transfers based on sequences of bed pressure images. The second algorithm analyzed the sagittal CP trajectory during SiSt transfers based on the bed pressure sequences. The third algorithm combined the bed and floor sensor outputs to measure the duration of SiSt transfers from the initial forward motion to a stable upright position. The three algorithms were able to successfully distinguish healthy and mobility-impaired transfers. The results of the algorithms were verified through video analysis by a clinical team as well as through comparisons to recent publications in related literature.

Given the challenge of interpreting and predicting human motion during ongoing smart home monitoring, context-awareness was introduced to the system design. Several sources of context were identified and an algorithm was designed to extract features from pressure-sensitive grab bars which could be incorporated into the mobility classification 
of the occupant. A simulated context-aware environment was then designed to observe the functionality of the smart home monitoring system. Results obtained by the algorithms during actual clinical trials were combined to create various occupant scenarios and the function of context-awareness in the monitoring system design was observed. The system presented in this thesis can be customized to suit the needs of a particular occupant and can be integrated into a larger framework which includes additional types of sensors and context-aware sources within the smart home environment.

\subsection{Contributions}

A monitoring system for seniors which integrated several different technologies into the smart home environment was introduced in Chapter 2 and has been published in [7]. Pressure sensing was also introduced in Chapter 2 as a form of ongoing monitoring and became the primary method of data collection throughout the thesis. A pressure-based smart home design has been published in [8] and a method of breathing signal analysis has been published in [9]. In Chapter 3, the pressure-based experimental setup and verification methods for mobility analysis were described and have been published in [10], [11] and [12].

An algorithm was presented in Chapter 4 which performed the detection and feature extraction of ROI from pressure image sequences during bed-exit. This algorithm was published in [13] and related preliminary work was previously published in [14]. The sagittal center $\mathrm{CP}$ trajectory was characterized by a feature extraction algorithm presented in Chapter 5 and submitted for review in [15]. An algorithm which measured the duration 
of the SiSt transfer using bed and floor pressure sequences was introduced in Chapter 6 and has been published in [16]. Additional analyses of the results of this algorithm were published in [17] and [18], along with related clinical implications.

Sources of context were introduced to the monitoring system in Chapter 7 including an algorithm for the extraction of clinical features from pressure-sensitive grab bars. The algorithm components were published in [19] and [20], the results of the clinical trials were published in [21] and related implications for the clinical field were published in [22]. A simulated context-aware environment was presented in Chapter 8 to demonstrate the function of the smart home monitoring system and has also been published in [23].

\subsection{Suggested Future Work}

The pressure sensing in this thesis was embedded into bed and grab bars to analyze body weight transfers as the assessment of functional mobility. Future work could investigate the addition of sensors in other home objects such as furniture, kitchen appliances or flooring. This could provide information as to the motion of an occupant throughout the smart home and highlight changes in their behaviour patterns over time.

Additional data should be collected from new clinical trials to verify that the promising results in this thesis can be interpolated to the entire. Patients should be included who exhibit mobility impairments which are easily perceived as well as patients who cover a wide range of more subtle impairments or levels of decline. Patients should be tested in smart home trials over extended periods of time to allow further observation of mobility trends. 
Future work could include an investigation into the data fusion and classifier designs to provide optimal decision making within the smart home environment. Automation of the algorithm selection and context inputs could be explored along with the feedback of relevant parameters back to the feature extraction level. Additional sources of context which may relate to mobility could also be investigated such as features obtained by light and temperature sensing, appliance usage, or the analysis of sounds within the home. Finally, the current system of mobility analysis could be incorporated into a larger framework which includes the monitoring of additional characteristics representative of the health and well-being of a smart home occupant. 


\section{References}

[1] Public Health Agency of Canada. (2010, June 29). Canada's Aging Population [Online]. Available: http://www.phac-aspc.gc.ca/seniors-aines/publications/public/various-varies/papier-fed-paper/indexeng.php.

[2] A. Fleury, M. Vacher, and N. Noury, "SVM-based multi-modal classification of activities of daily living in health smart homes: sensors, algorithms and first experimental results," IEEE Trans. Inf. Technol. Biomed., vol. 14, no. 2, pp. 274-283, Mar. 2010.

[3] M. Guennoun and K. El-Khatib, "Securing medical data in smart homes," in Proc. IEEE Int. Workshop Medical Meas. and Applicat., Cetraro, Italy, 2009, pp. 104-107.

[4] C. Rougier, J. Meunier, A. St-Arnaud, and J. Rousseau, "Monocular 3D head tracking to detect falls of elderly people," in Proc. $28^{\text {th }}$ Int. Conf. IEEE Eng. Med. Biol. Soc., New York, NY, USA, 2006, pp. 6384-6387.

[5] C. N. Scanaill, B. Ahearne, and G. M. Lyons, "Long-term telemonitoring of mobility trends of elderly people using SMS messaging," IEEE Trans. Inf. Technol. Biomed., vol. 10, no. 2, pp. 412-413, Apr. 2006.

[6] D. Lai, R. Begg, and M. Palaniswami, "Computational intelligence in gait research: a perspective on current applications and future challenges," IEEE Trans. Inf. Technol. Biomed., vol. 13, no. 5, pp. 687-702, Sep. 2009.

[7] A. Arcelus, M. H. Jones, R. Goubran, and F. Knoefel, "Integration of smart home technologies in a health monitoring system for the elderly," in Proc. $21^{\text {st }}$ Int. Conf. Advanced Inform. Networking and Applicat. Workshops, Niagara Falls, ON, Canada, 2007, pp. 820-825.

[8] M. H. Jones, A. Arcelus, R. A. Goubran, and F. Knoefel, "A pressure sensitive home environment," in Proc. IEEE Int. Workshop Haptic Audio Visual Environments Applicat., Ottawa, ON, Canada, 2006, pp. 10-14.

[9] M. Holtzman, A. Arcelus, R. Goubran, and F. Knoefel, "Breathing signal fusion in pressure sensor arrays," in Proc. IEEE Int. Workshop Medical Meas. and Applicat., Ottawa, ON, Canada, 2008, pp. 71-76.

[10] D. Blaney, D. Cloutier, K. Irvine, K. Jensen, A. Arcelus, F. Knoefel, R. Goubran, H. Sveistrup, and M. Bilodeau, "Development of a checklist to differentiate stand-to-sit bed transfer patterns between healthy older adults and those who had a stroke or hip fracture," presented at the $30^{\text {th }}$ Annu. Scientific Meeting Canadian Geriatrics Soc., Ottawa, ON, Canada, 2010. 
[11] J. Medwid, O. Habib, A. Arcelus, M. Bilodeau, F. Knoefel, R. Goubran, and H. Sveistrup, "The Ottawa sit-to-stand checklist for task analysis," presented at the $30^{\text {th }}$ Annu. Scientific Meeting Canadian Geriatrics Soc., Ottawa, ON, Canada, 2010.

[12] H. Sveistrup, M. Fitzpatrick, J. McLeod, O. Habib, A. Arcelus, M. Bilodeau, R. Goubran, and F. Knoefel, "Clinical populations can be distinguished on sit-to-stand movement parameters," to be presented at the ACRM ASNR Joint Educ. Conf. Progress Rehabil. Res., Montreal, QC, Canada, 2010.

[13] A. Arcelus, I. Veledar, R. Goubran, F. Knoefel, H. Sveistrup, and M. Bilodeau, "Measurements of sitto-stand timing and symmetry from bed pressure sensors," to appear in IEEE Trans. Instrum. Meas. May 2011.

[14] I. Veledar, A. Arcelus, R. Goubran, F. Knoefel, H. Sveistrup, and M. Bilodeau, "Sit-to-stand timing measurements using pressure sensitive technology," in Proc. IEEE Int. Instrum. Meas. Technology Conf., Austin, TX, USA, 2010, pp. 1337-1340.

[15] A. Arcelus, R. Goubran, H. Sveistrup, M. Bilodeau and F. Knoefel, "Context-aware smart home monitoring through pressure measurement sequences," submitted to IEEE Trans. Instrum. Meas. Jul. 2010.

[16] A. Arcelus, C. L. Herry, R. A. Goubran, F. Knoefel, H. Sveistrup, and M. Bilodeau, "Determination of sit-to-stand transfer duration using bed and floor pressure sequences," IEEE Trans. Biomed. Eng., vol. 56, no. 10, pp. 2485-2492, Oct. 2009.

[17] F. Knoefel, A. Arcelus, R. Goubran, M. Bilodeau, and H. Sveistrup, "Can a mat sensor algorithm identify mobility challenges in older adults getting out of bed?," in Proc. $7^{\text {th }}$ World Conf. Int. Soc. for Gerontechnology, Vancouver, BC, Canada, 2010, pp. 225.

[18] S. Mondoux, P. Carlson, N. Foubert, A. Arcelus, F. Knoefel, R. Goubran, M. Bilodeau, and H. Sveistrup, "Smart mat technology: can it differentiate bed transfers in older healthy and older hip fracture patients?," presented at the Annu. General Meeting Regional Geriatrics Program, Ottawa, ON, Canada, 2009.

[19] A. Arcelus, M. Holtzman, I. Veledar, R. Goubran, H. Sveistrup, and P. Guitard, "Contact location estimation from a nonlinear array of pressure sensors," in Proc. IEEE Int. Instrum. Meas. Technology Conf., Victoria, BC, Canada, 2008, pp. 1969-1973.

[20] M. Holtzman, A. Arcelus, I. Veledar, R. Goubran, H. Sveistrup, and P. Guitard, "Force estimation with a non-uniform pressure sensor array," in Proc. IEEE Int. Instrum. Meas. Technology Conf., Victoria, BC, Canada, 2008, pp. 1974-1979.

[21] A. Arcelus, M. Holtzman, R. Goubran, H. Sveistrup, P. Guitard, and F. Knoefel, "Analysis of commode grab bar usage for the monitoring of older adults in the smart home environment," in Proc.

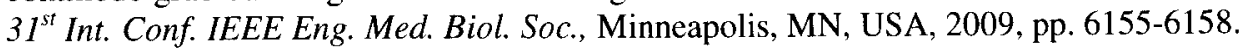

[22] M. Kennedy, A. Arcelus, P. Guitard, R. Goubran, F. Knoefel, and H. Sveistrup, "Using a commode changes how older adults, adults post-stroke and adults post-hip-fracture sit and stand from a toilet," presented at the $30^{\text {th }}$ Annu. Scientific Meeting Canadian Geriatrics Soc., Ottawa, ON, Canada, 2010. 
[23] A. Arcelus, R. Goubran, F. Knoefel, H. Sveistrup, and M. Bilodeau, "Context-aware smart home monitoring through pressure measurement sequences," Proc. IEEE Int. Workshop Medical Meas. and Applicat., Ottawa, ON, Canada, 2010, pp. 32-37.

[24] S. Junnila, H. Kailanto, J. Merilahti, A. Vainio, A. Vehkaoja, M. Zakrzewski, and J. Hyttinen, "Wireless, multipurpose in-home health monitoring platform: two case trials," IEEE Trans. Inf. Technol. Biomed., vol. 14, no. 2, pp. 447-455, Mar. 2010.

[25] P. Rashidi, D. Cook, "Keeping the resident in the loop: adapting the smart home to the user," IEEE Trans. Syst. Man, Cybern. A, Syst., Humans, vol.39, no. 5, pp. 949-959, Sep. 2009.

[26] C. Lu and L. Fu, "Robust location-aware activity recognition using wireless sensor network in an attentive home," IEEE Trans. Automation Sci. and Eng., vol. 6, no. 4, pp. 598-609, Oct. 2009.

[27] H. H. Kim, K. N. Ha, S. Lee, and K. C. Lee, "Resident location-recognition algorithm using a Bayesian classifier in the PIR sensor-based indoor location-aware system," IEEE Trans. Syst. Man Cybern. C, Appl. Rev., vol. 39, no. 2, pp. 240-245, Mar. 2009.

[28] O. Brdiczka, J. L. Crowley, and P. Reignier, "Learning situation models in a smart home," IEEE Trans. Syst. Man Cybern. B, Cybern., vol. 39, no. 1, pp. 56-63, Feb. 2009.

[29] H. K. Pung, T. Gu, W. Xue, P. P. Palmes, J. Zhu, W. L. Ng, C. W. Tang, and N. H. Chung, "Contextaware middleware for pervasive elderly homecare," IEEE J. Sel. Areas Commun., vol. 27, no. 4, pp. 510-524, May 2009.

[30] F. Chiti, R. Fantacci, F. Archetti, E. Messina, and D. Toscani, "An integrated communications framework for context aware continuous monitoring with body sensor networks," IEEE J. Sel. Areas Commun., vol. 27, no. 4, pp. 379-386, May 2009.

[31] F. Knoefel, L. Patrick, R. Goubran, M. Borduas, J. Tetro, and J. Morley, "TAFETA research program: reducing falls-related injuries," presented at the Celebrating Innovations in Healthcare Expo, Toronto, ON, Canada, 2008.

[32] W. Janssen, S. Külcü, H. Horemans, H. Stam, and J. Bussmann, "Sensitivity of accelerometry to assess balance control during sit-to-stand movement," IEEE Trans. Neural Syst. Rehabil. Eng., vol. 16 , no. 5 , pp. 479-484, Oct. 2008.

[33] S. Lord, S. Murray, K. Chapman, B. Munro, and A. Tiedemann, "Sit-to-stand performance depends on sensation, speed, balance, and psychological status in addition to strength in older people," $J$. of Gerontology: Medical Sci., vol. 57A, no. 8, pp. 539-543, Aug. 2002.

[34] L. C. Walter, L. Lui, C. Eng, and K. Covinsky, "Risk of hip fracture in disabled community-living older adults," J. of the Amer. Geriatrics Soc., vol. 62, no. 11, pp. 2145-56, Jan. 2003.

[35] M. Narayanan, S. Redmond, M. Scalzi, S. Lord, B. Celler, and N. Lovell, "Longitudinal falls-risk estimation using triaxial accelerometry," IEEE Trans. Biomed. Eng., vol. 57, no. 3, pp. 534-541, Mar. 2010.

[36] S. W. Muir, K. Berg, B. Chesworth, and M. Speechley, "Use of the Berg balance scale for predicting multiple falls in community-dwelling elderly people: a prospective study," Physical Therapy, vol. 88, no. 4, pp. 449-459, Apr. 2008. 
[37] M. Goffredo, M. Schmid, S. Conforto, M. Carli, A. Neri, and T. D. Alessio, "Markerless human motion analysis in Gauss-Laguerre domain: application to sit-to-stand in young and elderly people," IEEE Trans. Inf. Technol. Biomed., vol. 13, no. 2, pp. 209-216, Mar. 2009.

[38] W. K. Durfee, L. Savard, and S. Weinstein, "Technical feasibility of teleassessments for rehabilitation," IEEE Trans. Neural Syst. Rehabil. Eng., vol. 15, no. 1, pp. 23-29, Mar. 2007.

[39] I. Veledar, "Patient bed transfer analysis using pressure sensor arrays," M.A.Sc. thesis, Dept. Sys. Comp. Eng., Carleton Univ., Ottawa, ON, Canada, 2009.

[40] P. O. Riley, D. E. Krebs, and R. A. Popat, "Biomechanical analysis of failed sit-to-stand," IEEE Trans. Neural Syst. Rehabil. Eng., vol. 5, no. 4, pp. 353-359, Dec. 1997.

[41] W. Mathiyakom, J. L. McNitt-Gray, P. Requejo, and K. Costa, "Modifying center of mass trajectory during sit-to-stand tasks redistributes the mechanical demand across the lower extremity joints," Clinical Biomechanics, vol. 20, no. 1, pp. 105-111, Jan. 2005.

[42] J. P. Scholz, D. Reisman, and G. Schöner, "Effects of varying task constraints on solutions to joint coordination in a sit-to-stand task," Experimental Brain Research, vol. 141, pp. 485-500, Oct. 2001.

[43] M. Lomaglio and J. Eng, "Muscle strength and weight-bearing symmetry relate to sit-to-stand performance in individuals with stroke," Gait \& Posture, vol. 22, no. 2, pp. 126-131, Aug. 2004.

[44] M. Anan, K. Okumura, N. Kito, and K. Shinkoda, "Effects of variation in cushion thickness on the sitto-stand motion of elderly people," J. Physical Therapy Sci., vol. 20, no. 1, pp. 51-57, Jan. 2008.

[45] C. Duclos, S. Nadeau, and J. Lecours, "Lateral trunk displacement and stability during sit-to-stand transfer in relation to foot placement in patients with hemiparesis," Neurorehabilitation and Neural Repair, vol. 22, no. 6, pp. 715-722, Nov. 2008.

[46] N. Bidargaddi, L. Klingbeil, A. Sarela, J. Boyle, V. Cheung, C. Yelland, M. Karunanithi, and L. Gray, "Wavelet based approach for posture transition estimation using a waist worn accelerometer," in Proc. $29^{\text {th }}$ Int. Conf. IEEE Eng. Med. Biol. Soc., Lyon, France, 2007, pp. 1884-1887.

[47] A. Salarian, H. Russmann, F. Vingerhoets, P. Burkhard, and K. Aminian, "Ambulatory monitoring of physical activities in patients with Parkinsons disease," IEEE Trans. Biomed. Eng., vol. 54, no. 12, pp. 2296-2299, Dec. 2007.

[48] B. Najafi, K. Aminian, F. Loew, Y. Leblanc, and P. Robert, "Measurement of stand-sit and sit-stand transitions using a miniature gyroscope and its application in fall risk evaluation in the elderly," IEEE Trans. Biomed. Eng., vol. 49, no. 8, pp. 843-851, Aug. 2002.

[49] B. Najafi, L. Aminian, A. Paraschiv Ionescu, F. Loew, C. Bula, and P. Robert, "Ambulatory system for human motion analysis using a kinematic sensor: Monitoring daily physical activity in the elderly," IEEE Trans. Biomed. Eng., vol. 50, no. 6, pp. 711-723, Jun. 2003.

[50] T. Yamada and S. Demura, "Relationships between ground reaction force parameters during a sit-tostand movement and physical activity and falling risk of the elderly and a comparison of the movement characteristics between the young and the elderly," Archives Gerontology and Geriatrics, vol. 48, no. 1, pp. 73-77, Jan. 2009. 
[51] W. Liau, W. Chu, and L. Fu, "Inhabitants tracking system in a cluttered home environment via floor load sensors," IEEE Trans. Automation and Sci. Eng., vol. 5, no. 1, pp. 10-20, Jan. 2008.

[52] M. H. Jones, R. A. Goubran, and F. Knoefel, "Identifying movement onset times for a bed-based pressure sensor array," in Proc. IEEE Int. Workshop Medical Meas. and Applicat., Benevento, Italy, 2006, pp. 111-114.

[53] M. H. Jones, R. A. Goubran, and F. Knoefel, "Nocturnal monitoring of elderly patients with a bedplaced pressure pad," presented at the Canadian Assoc. Gerontology Conf., Halifax, NS, Canada, 2005 .

[54] M. H. Jones, R. A. Goubran, and F. Knoefel, "Reliable respiratory rate estimation from a bed pressure array," in Proc. $28^{\text {th }}$ Int. Conf. IEEE Eng. Med. Biol. Soc., New York, NY, USA, 2006, pp. 64106413.

[55] L. Saint-Bauzel, V. Pasqui, and I. Monteil, "A reactive robotized interface for lower limb rehabilitation: clinical results," IEEE Trans. Robot., vol. 25, no. 3, pp. 583-592, Jun. 2009.

[56] D. J. van den Heever, K. Schreve, and C. Scheffer, "Tactile sensing using force sensing resistors and a super-resolution algorithm," IEEE Sensors J., vol. 9, no. 1, pp. 29-35, Jan. 2009.

[57] V. Egorov and A. P. Sarvazyan, "Mechanical imaging of the breast," IEEE Trans. Med. Imag., vol. 27, no. 9, pp. 1275-1287, Sep. 2008.

[58] V. Egorov, S. Ayrapetyan, and A. P. Sarvazy, "Prostate mechanical imaging: 3-D image composition and feature calculations," IEEE Trans. Med. Imag., vol. 25, no. 10, pp. 1329-1340, Oct. 2006.

[59] H. Yu, C. Caldwell, K. Mah, and D. Mozeg, "Coregistered FDG PET/CT-based textural characterization of head and neck cancer for radiation treatment planning," IEEE Trans. Med. Imag., vol. 28, no. 3, pp. 374-383, Mar. 2009.

[60] A. Gholipour, N. Kehtarnavaz, R. Briggs, M. Devous, and K. Gopinath, "Brain functional localization: a survey of image registration techniques," IEEE Trans. Med. Imag., vol. 26, no. 4, pp. 427-451, Apr. 2007.

[61] J. Manikandan, B. Venkataramani, and M. Jayachandran, "Evaluation of edge detection techniques towards implementation of automatic target recognition," in Proc. Int. Conf. Computational Intell. and Multimedia Applicat., Sivakasi, India, 2007, pp. 441-445.

[62] P. Mlsna and J. Rodriguez, "Gradient and Laplacian edge detection," in Handbook of Image and Video Process., $2^{\text {nd }}$ ed., Burlington, USA: Elsevier Academic Press, 2005, ch. 4, sec. 13, pp. 535-548.

[63] Y. Luo and R. Duraiswami, "Canny edge detection on NVIDIA CUDA," in Proc. IEEE Comput. Society Conf. Comput. Vision and Pattern Recognition Workshops, Anchorage, Alaska, USA, 2008, pp. 1-8.

[64] R. Dufour, E. Miller, and N. Galatsanos, "Template matching based object recognition with unknown geometric parameters," IEEE Trans. Image Process., vol. 11, no.12, pp. 1385-1396, Dec. 2002.

[65] J. Lerga, M. Vrankic, and V. Sucic, "A signal denoising method based on the improved ICI rule," IEEE Signal Process. Lett., vol. 15, pp. 601-604, 2008. 
[66] S. Sriram, S. Nitin, K. M. M. Prabhu, and M. J. Bastiaans, "Signal denoising techniques for partial discharge measurements," IEEE Trans. Dielectr. Electr. Insul. vol. 12, no.6, pp. 1182-1191, Dec. 2005 .

[67] P. Bouboulis, K. Slavakis, and S. Theodoridis, "Adaptive kernel-based image denoising employing semi-parametric regularization," IEEE Trans. Image Process., vol. 19, no.6, pp. 1465-1479, Jun. 2010.

[68] H. Rabbani, R. Nezafat, and S. Gazor, "Wavelet-domain medical image denoising using bivariate Laplacian mixture model," IEEE Trans. Biomed. Eng., vol. 56, no. 12, pp. 2826-2837, Dec. 2009.

[69] H. C. Powell Jr., M. A. Hanson, and J. Lach, "On-body inertial sensing and signal processing for clinical assessment of tremor," IEEE Trans. Biomed. Circuits Syst., vol. 3, no. 2, pp. 108-116, Apr. 2009.

[70] F. Öhberg, H. Grip, U. Wiklund, Y. Sterner, J. Stefan Harlsson, and B. Gerdle, "Chronic whiplash associated disorders and neck movement measurements: an instantaneous helical axis approach," IEEE Trans. Inf. Technol. Biomed., vol. 7, no. 4, pp. 274-282, Dec. 2003.

[71] J. B. Tilbury, W. J. Van Eetvelt, J. M. Garibaldi, J.S. H. Curnsw, and E. C. Ifeachor, "Receiver operating characteristic analysis for intelligent medical systems - a new approach for finding confidence intervals," IEEE Trans. Biomed. Eng., vol. 47, no. 7, pp. 952-963, Jul. 2000.

[72] K. Shafqat, S. K. Pal, P. A. Kyriacou, "Evaluation of two detrending techniques for application in heart rate variability," Proc. $29^{\text {th }}$ Int. Conf. IEEE Eng. Med. Biol. Soc., Lyon, France, 2007, pp. 267270.

[73] M. P. Tarvainen, P. O. Ranta-aho, P. A. Karjalainen, "An advanced detrending method with application to HRV analysis," IEEE Trans. Biomed. Eng., vol. 49, no. 2, pp.172-175, Feb. 2002.

[74] S. Vergura, G. Accianti, V. Amoruso, G. Patrono and F. Vacca, "Descriptive and inferential statistics for supervising and monitoring the operation of PV plants," IEEE Trans. Ind. Electron., vol. 56, no.11, pp. 4456-4464, Nov. 2009.

[75] W. Janssen, H. Bussmann, and H. Stam, "Determinants of the sit-to-stand movement: a review," Physical Therapy, vol. 82, no. 9, pp. 866-879, Sep. 2002.

[76] D. Hosmer and S. Lemeshow, "Multiple Logistic Regression," in Appl. Logistic Regression, $2^{\text {nd }}$ ed. Hoboken, USA: John Wiley \& Sons, Inc., 2004, ch. 2, sec. 2, pp. 31-40.

[77] H. Hirschfeld, M. Thorsteinsdottir, and E. Olsson, "Coordinated ground forces exerted by buttocks and feet are adequately programmed for weight transfer during sit-to-stand," J. Neurophysiology, vol. 82, no. 6, pp. 3021-3029, Dec. 1999.

[78] B. Lindsay, "Randomized controlled trials of socially complex nursing interventions: creating bias and unreliability?" J. Advanced Nursing, vol. 45, no. 1, pp. 84-94, Jan. 2004.

[79] G. Bornhöft, S. Maxion-Bergemann, U. Wolf, G. Kienle, A. Michalsen, H. Vollmar, S. Gilbertson, and P. Matthiessen, "Checklist for the qualitative evaluation of clinical studies with particular focus on external validity and model validity," BMC Medical Research Methodology, vol. 6, pp. 56-68, Dec. 2006. 
[80] L. Ljung, System Identification: Theory for the User, 2 ed., Englewood Cliffs, NJ: Prentice-Hall, 1987.

[81] M. Wachowiak, G. Rash, P. Quesada, and A. Desoky, "Wavelet-based noise removal for biomechanical signals: a comparative study mark," IEEE Trans. Biomed. Eng., vol. 47, no. 3, pp. 260268, Mar. 2000.

[82] A. Cohen, I. Daubechies, and J. Feauveau, "Biorthogonal bases of compactly supported wavelets," Commun. Pure Appl. Math., vol. 45, no. 5, pp. 485-560, Jun. 1992.

[83] M. Unser, "Splines: a perfect fit for signal and image processing," IEEE Signal Processing Mag., vol. 16, no. 6, pp. 22-38, Nov. 1999.

[84] L. Xiang, L. Feng, L. Zhou, and Y. Shi, "Learning in an ambient intelligent world: enabling technologies and practices," IEEE Trans. Knowl. Data Eng., vol. 21, no. 6, pp. 910-924, Jun. 2009.

[85] K. Henricksen and J. Indulska, "Modelling and using imperfect context information," in Proc. $2^{\text {nd }}$ IEEE Annu. Conf. Pervasive Computing and Commun. Workshops, Orlando, FL, USA, 2004, pp. 3337.

[86] R. Wishart, K. Henricksen, and J. Indulska, "Context privacy and obfuscation supported by dynamic context source discovery and processing in a context management system," in Proc. $4^{\text {th }}$ Int. Conf. Ubiquitous Intell. Computing, Hong Kong, China, 2007, pp. 929-940.

[87] E. Mattila, J. Pärkkä, M. Hermersdorf, J. Kaasinen, J. Vainio, K. Samposalo, J. Merilahti, J. Kolari, R. Lappalainen and I. Korhonen, "Mobile diary for wellness management - results on usage and usability in two user studies," IEEE Trans. Inf. Technol. Biomed., vol. 12, no. 4, pp. 501-512, Jul. 2008.

[88] B. Rhodes, "Using physical context for just-in-time information retrieval," IEEE Trans. Comp.,, vol. 52, no. 8, pp. 1011-1014, Aug. 2003.

[89] S. Lelievre and K. Schultz, "Does computer use in patient-physician encounters influence patient satisfaction?," Canadian Family Physician, vol. 56, no. 1, pp. e6-e12, Jan..

[90] 2010Canada Health Infoway Inc. (2010, June 29). 2015: Advancing Canada's Next Generation of Healthcare [Online]. Avalailable: http://www.infoway-inforoute.ca/lang-en/working-withehr/resource-centre.

[91] H. Sveistrup, D. Lockett, N. Edwards, and F. Aminzadeh, "Evaluation of bath grab bar placement for older adults," Technol. Disability, vol. 18, no. 2, pp. 45-87, Jul. 2006. 